\title{
Understanding How Developers Reuse Stack Overflow Code in Their GitHub Projects
}

by

Razieh Tekieh

A Thesis submitted to

the Faculty of Graduate Studies and Research

in partial fulfilment of

the requirements for the degree of

Master of Computer Science

Department of Computer Science

Carleton University

Ottawa, Ontario, Canada

August 2021

Copyright (C)

2021 - Razieh Tekieh 
The undersigned recommend to

the Faculty of Graduate Studies and Research

acceptance of the Thesis

\title{
Understanding How Developers Reuse Stack Overflow Code in Their GitHub Projects
}

\author{
Submitted by Razieh Tekieh \\ in partial fulfilment of the requirements for the degree of \\ Master of Computer Science
}

Dr. Olga Baysal, Supervisor

Dr. Mehrdad Sabetzadeh, External Examiner

$\overline{\text { Dr. Rabe Abdalkareem, Internal Examiner }}$

Dr. Alan Tsang, Chair of Defence

Dr. Michel Barbeau, Department Chair

Carleton University

2021 


\section{Abstract}

Stack Overflow and other popular Q\&A forums include a variety of reusable code snippets for software developers. Instead of writing new code, most software developers prefer to reuse existing code which in a software projects, this reuse of code is referred to as "code cloning". In this study we look into how software developers reused and adopted code snippets from Stack Overflow in projects hosted on GitHub. To achieve our goal, we create a code pair dataset that maps Stack Overflow code snippets to GitHub commits with the help of SOTorrent and GHCodeSnippetHistory. Our dataset consists of code pairs from four programming languages including Java, JavaScript, PHP, and Python.

The first part of the study concentrates on finding clones between Stack Overflow and GitHub code snippets and its challenges. The result of first part indicates around $77 \%$ of code pairs do not have clone. However, around $15.8 \%$ of code pairs have Type I, $5.89 \%$ and $11.71 \%$ have Type II and III, respectively. Based on these findings, developers are more likely to integrate Stack Overflow code snippets rather than use them directly in their projects. However, if developers reuse code snippets, they more prefer to copy\&paste them to make modifications.

In the second part we develop and test multi-label classifiers for predicting the types of code clones for four feature sets. The extracted feature sets are based on TF-IDF, Code2Vec, and Word2Vec models in addition to the statistical feature set derived from the programming paradigms. The results of applying classifiers to our 
extracted feature sets reveal that most of our models are likely overfit for the majority class. Also, statistical feature set was slightly better than other feature set. For this feature set, all multi-label classification techniques which used Random Forest as based algorithms were able to predict clone types with a F1-Score more than $76 \%$. 


\section{Acknowledgments}

Two years after starting my Master's program, now I'm pleased to write the last note in my thesis. This research work expanded my expertise on a very interesting aspect of computer science dealing with software engineering, mining software repositories, and data science. I would like to mention the people who have continuously supported and directed me all the way through my graduate school journey.

My special thanks go to my research supervisor, Dr. Olga Baysal, for providing me with this great opportunity to work under her supervision. I am sincerely thankful for all of her support, attention, kindness and encouraging spirit. This research would not be possible to conduct without her ongoing help.

I am also grateful to the thesis committee members: Dr. Rabe Abdalkareem and Dr. Mehrdad Sabetzadeh for their time and invaluable feedback on my research work. Besides, I would like to acknowledge the help of Nathaniel Salami in evaluating iClones results. And also I am thankful to my peer Saraj Manes for his help and support.

Moreover, I take this opportunity to highly thank my parents for all of their endless support and encouragement through my life. 


\section{Table of Contents}

Abstract iii

\begin{tabular}{|l|l|}
\hline Acknowledgments & v
\end{tabular}

Table of Contents $\quad$ vi

List of Tables $\quad$ x

List of Figures $\quad$ xii

Nomenclature xiii

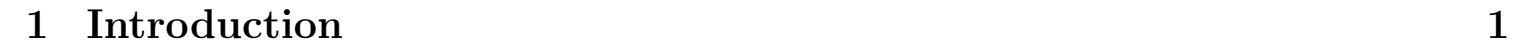

1.1 Code Reuse . . . . . . . . . . . . . . . . . . . . 1

1.2 Motivation . . . . . . . . . . . . . . . . . . . . . . 3

1.3 Research Questions . . . . . . . . . . . . . . . . . . . 5

1.4 Contributions . . . . . . . . . . . . . . . . . . . 7

1.5 Organization of the Thesis $\ldots \ldots \ldots \ldots$. . . . . . . . . . . 7

\begin{tabular}{|lll}
\hline 2 & Background and Related Work & 8
\end{tabular}

2.1 Code Reuse . . . . . . . . . . . . . . . . . . . . . . . . . . . . . . 8

2.2 Code Clone Types $\ldots \ldots \ldots$. . . . . . . . . . . . . . . . . . . 10

$2.2 .1 \quad$ Type I Code Clones . . . . . . . . . . . . . . . . . . . . . . . . 11 
2.2 .2 Type II Code Clones $\ldots \ldots \ldots \ldots$

2.2 .3 Type III Code Clones . . . . . . . . . . . . . . . . . . . . . 12

$2.2 .4 \quad$ Type IV Code Clones . . . . . . . . . . . . . . . . . . . . 13

2.3 Code Clone Detection Techniques . . . . . . . . . . . . . . . 14

$2.4 \quad$ Classification Problem . . . . . . . . . . . . . . . . . . . . . . 22

$2.5 \quad$ Mining Software Repositories $\ldots \ldots \ldots \ldots \ldots$

$2.6 \quad$ Code Reuse in Mining Software Repositories . . . . . . . . . . . 26

2.7 Source Code as Natural Language $\ldots \ldots \ldots \ldots$

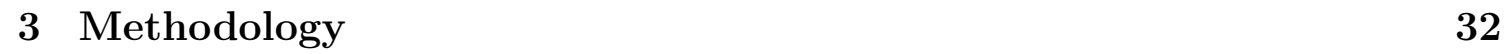

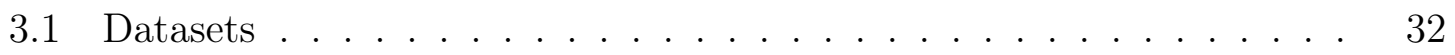

3.2 Code Clone Detection . . . . . . . . . . . . . . . . . . . . . . 39

3.2 .1 Overview of the Tools Used . . . . . . . . . . . . . . 40

3.2 .2 Manual Assessment of Code Clone Detection . . . . . . . . . 42

3.2 .3 Clone Detection Using IClones . . . . . . . . . . . . . . . 44

3.2.3.1 Preparing the Source Code . . . . . . . . . . . . . 44

$3.2 .3 .2 \quad$ Source Code Cleanup . . . . . . . . . . . . . . . 45

3.2 .3 .3 Running IClONES . . . . . . . . . . . . . . . . 45

3.2.3.4 Evaluating IClONES Results. . . . . . . . . . . . 46

$3.3 \quad$ Predicting Code Clone Types $\ldots \ldots \ldots \ldots$. . . . . . . . . 47

$3.3 .1 \quad$ Preparing Dataset $\ldots \ldots \ldots \ldots$. . . . . . . . . . . 47

3.3 .2 Data Cleaning And Pre-processing . . . . . . . . . . . . . 48

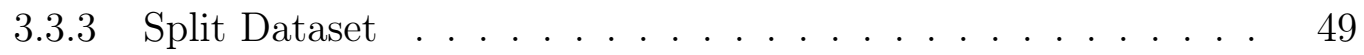

3.3 .4 Feature Extraction . . . . . . . . . . . . . . 50

3.3.4.1 Statistical Features . . . . . . . . . . . . . . 51

3.3 .4 .2 TF-IDF $\ldots \ldots \ldots \ldots \ldots \ldots \ldots \ldots \ldots$

$3.3 .4 .3 \quad$ Word Embeddings $\ldots \ldots \ldots \ldots$ 
3.3 .5 Multi-label Classification Models . . . . . . . . . . . . 54

3.3 .6 Balancing Multi-label Classification . . . . . . . . . . 58

3.3 .7 Evaluation Metrics . . . . . . . . . . . . . . . . 59

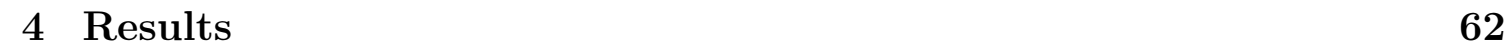

4.1 Code Clone Detection . . . . . . . . . . . . . . . . . . 62

4.1.1 RQ1: How Accurately Can Clone Detection Tools Identify Code Clones Between Stack Overflow and GitHub? . . . . . . . . 63

4.1.2 RQ2: What Types of Clones Are Present in the Code Snippets Reused From Stack Overflow in the GitHub Projects? . . . . . 68

4.2 Code Clone Prediction . . . . . . . . . . . . . . . . . . . . . . 72

$4.2 .1 \quad$ Dataset Overview After Pre-processing and Splitting _. . . . 73

$4.2 .2 \quad$ Feature Sets . . . . . . . . . . . . . . . . . . . . . 75

4.2 .3 Balancing . . . . . . . . . . . . . . . . . 77

4.2.4 RQ3: How Accurately Can We Predict the Clone Type Given Two Code Snippets? . . . . . . . . . . . . . . . . . . . . . . . 79

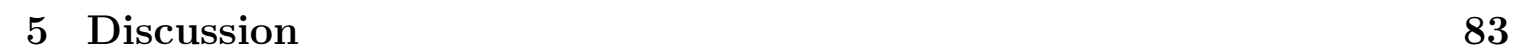

5.1 Findings $\ldots \ldots \ldots \ldots \ldots \ldots$

5.2 Implications $\ldots \ldots \ldots \ldots \ldots \ldots \ldots \ldots \ldots \ldots \ldots$

$5.2 .1 \quad$ Software Developers $\ldots \ldots \ldots \ldots$

5.2 .2 Researchers . . . . . . . . . . . . . . . . . . . . 88

5.2 .3 Online Programming Platforms $\ldots \ldots \ldots$

5.3 Threats and Limitations $\ldots \ldots \ldots$

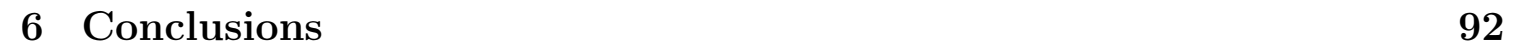

$6.1 \quad$ Summary of Contributions $\ldots \ldots \ldots \ldots$. . . . . . . . . . . . . 92

6.2 Future Work . . . . . . . . . . . . . . . . . . . . . . . . . . 94 
\begin{tabular}{ll}
\hline List of References & 96
\end{tabular}

\begin{tabular}{ll}
\hline Appendix A & 107
\end{tabular}

A.1 $\quad$ IClONES Configuration Results . . . . . . . . . . . . . . . . 107

A.2 Models' Results . . . . . . . . . . . . . . . . . . . . . . . 107 


\section{List of Tables}

$2.1 \quad$ Code clone types and their definitions. . . . . . . . . . . . . 11

$2.2 \quad$ An example of Type I code clone. . . . . . . . . . . . . . . . 11

$2.3 \quad$ An example of Type II code clone. . . . . . . . . . . . . . . . 12

$2.4 \quad$ An example of Type III code clone. . . . . . . . . . . . . . 13

$2.5 \quad$ An example of Type IV code clone. . . . . . . . . . . . . 13

$2.6 \quad$ Code clone detection techniques and tools. . . . . . . . . . . 17

2.7 Code reuse in the MSR research field. . . . . . . . . . . . . 27

3.1 The number of code pairs for each programming language in the dataset. 38

$3.2 \quad$ A general overview of code pairs in terms of character count. . . . . . 40

3.3 Statistical features. . . . . . . . . . . . . . . . . . . . . . . 52

4.1 Summary of the manual clone detection process. . . . . . . . . . 63

$4.2 \quad$ Similarity ratio of ICLONES with manually detected clones by human

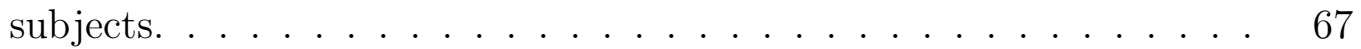

$4.3 \quad$ Evaluation of the clone detection results on 60 randomly selected samples. 68

4.4 Summary of IClONES' clone detection results. . . . . . . . . . . . 69

$4.5 \quad$ Distribution of multi-clone types in our sample. . . . . . . . . . 71

$4.6 \quad$ Distribution of clone types for each programming language after preprocessing. . . . . . . . . . . . . . . . . . . 73

4.7 The number of samples in the train and test sets for each label. . . . 74

4.8 Label distribution in the train and test sets. . . . . . . . . . 74 
4.9 Range of statistical features. . . . . . . . . . . . . . . . . . . 75

4.10 Top 100 selected words by TfidfVectorizer. . . . . . . . . . . . . . . 76

4.11 Number of words that do not exist in the pre-trained models. . . . . . 77

4.12 Distribution of labels in the balanced train sets. . . . . . . . . . . . . 78

4.13 Models' F1-score and hamming loss values for OriginalDB, BalancedDS1, BalancedDS2, and BalancedDS3. . . . . . . . . . . . . . . 81

4.14 F-score of statistical features for Random Forest based classifiers. . . 82

A.1 ICLONES results for different configuration over selected samples. . . 108

A.2 Precision, recall, F1-score, hamming loss, accuracy, and execution time over OriginalDS. . . . . . . . . . . . . . . . . . . . . . . . . . . . . . 109

A.3 Precision, recall, F1-score, hamming loss, accuracy, and execution time over BalancedDS1. . . . . . . . . . . . . . . . . . . . . . . . 110

A.4 Precision, recall, F1-score, hamming loss, accuracy, and execution time over BalancedDS2. . . . . . . . . . . . . . . . . . . . . . . . 111

A.5 Precision, recall, F1-score, hamming loss, accuracy, and execution time over BalancedDS3. . . . . . . . . . . . . . . . . . . . . . . . . . . . . 112

A.6 $\quad$ F1-score results per clone types over OriginalDS. . . . . . . . . . . . . 113

A.7 $\quad$ F1-score results per clone types over BalancedDS1. . . . . . . . . . . 114

A.8 $\quad$ F1-score results per clone types over BalancedDS2. . . . . . . . . . . 115

A.9 $\quad$ F1-score results per clone types over BalancedDS3. . . . . . . . . . . 116 


\section{List of Figures}

1.1 Our research roadmap. . . . . . . . . . . . . . . . . . 5

3.1 SOTorrent dataset layout $|1| . \ldots \ldots \ldots$. . . . . . . . . . . . 34

3.2 GHCodeSnippetHistory dataset layout $|2|$. . . . . . . . . . . . . . 35

3.3 Data collection: adding a Stack Overflow snippet to the GHCodeSnippetHistory dataset. . . . . . . . . . . . . . . . . . . 36

3.4 The workflow of creating our dataset. . . . . . . . . . . . . . . . . . 38

3.5 A sample commit $[2 \mid . \ldots \ldots$. . . . . . . . . . . . . . . . 39

3.6 Clone detection process. . . . . . . . . . . . . . . . . . . . 41

3.7 Manual clone detection process. . . . . . . . . . . . . . . . . . . . 43

3.8 Overview of the process of predicting code clone types. . . . . . . . . 47

3.9 Dataset schema in Pandas. . . . . . . . . . . . . . . . . . . . . . . 48

4.1 Ratio of detected clones for different configurations of ICLONES. . . . 65

4.2 Number of detected clones in Java samples. . . . . . . . . . . . . . . 65

4.3 Number of detected clones in JavaScript samples. . . . . . . . . . . . 65

$4.4 \quad$ Number of detected clones in PHP samples. . . . . . . . . . . . . . . 66

4.5 Number of detected clones in Python samples. . . . . . . . . . . . . . 66

4.6 Ratio of detected clones by IClONES. . . . . . . . . . . . . . . 70

4.7 Ratio of detected clones for each programming language. . . . . . . . 70

4.8 Distribution of labels. . . . . . . . . . . . . . . . . . . . . . . . . . . . . . 71 


\section{Nomenclature}

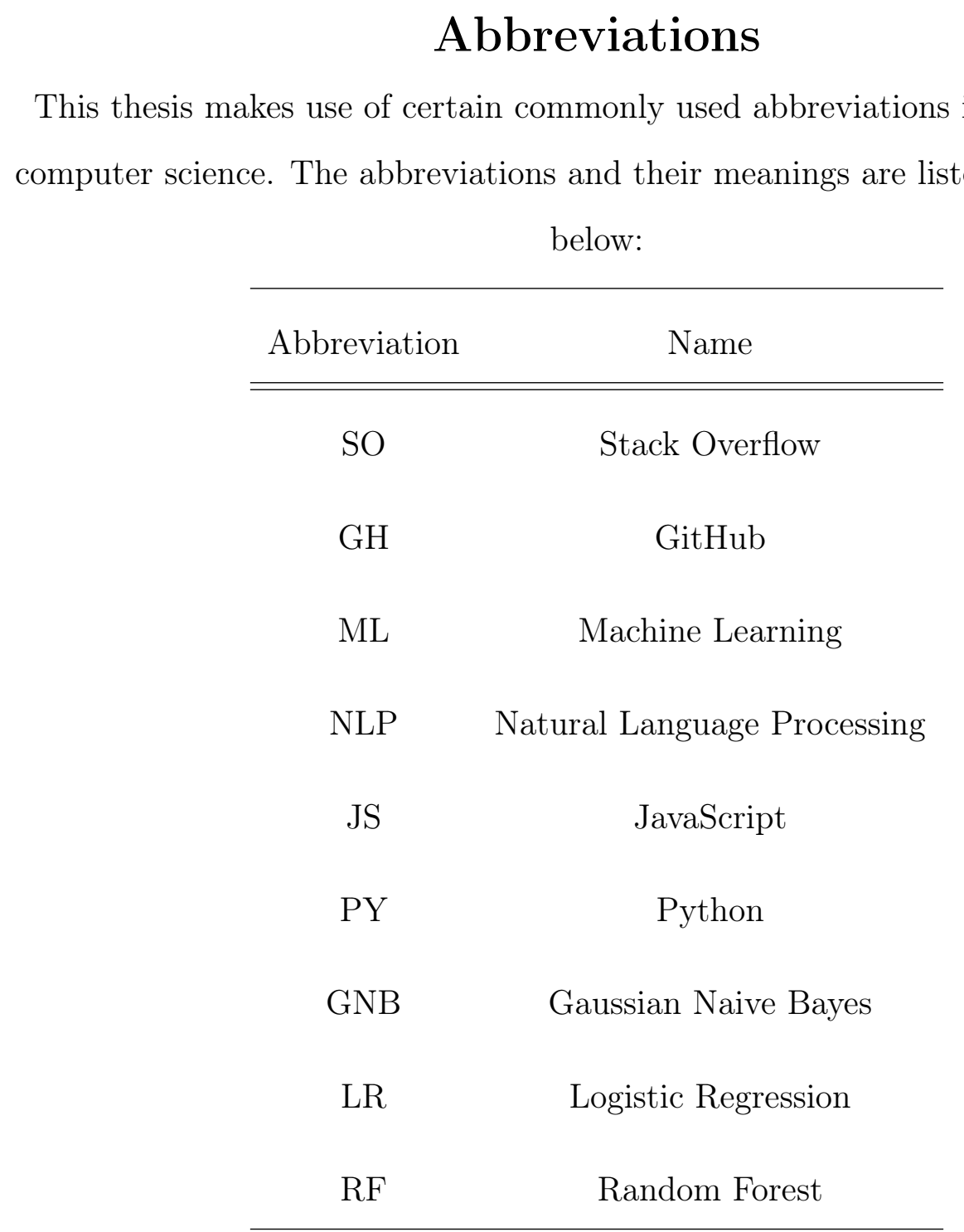




\section{Chapter 1}

\section{Introduction}

In this chapter, Section 1.1 describes the goal of this thesis and highlights the importance of studying code reuse. Section 1.2 explains the motivation behind this work. Section 1.3 outlines the research questions which this study tries to address, while Section 1.4 summarizes the key contributions of this work. Finally, Section 1.5 presents the organization of this thesis.

\subsection{Code Reuse}

Most software developers prefer to copy-paste program code instead of writing new code from scratch. In other words, they reuse code or code fragments (i.e., snippets) available on the web or from in-house developed projects in their own projects. A code snippet is a small chunk of the source code designed for a specific purpose such as a loop or a feature. In a software system, code clones are defined as similar or identical fragments of code, and "code cloning" refers to this reuse of code. Code cloning has both advantages and disadvantages which are still debatable. On the one hand, as writing new codes consumes considerable amount of time and effort, developers frequently search for code snippets on the other versions of a software or on the web in order to write code quickly and easily. From this perspective, code 
clones are beneficial. On the other hand, if a reused code snippet contains a bug (i.e., software defect), all the copied instances must be checked for this bug. That is to say, the code reuse may lead to bug propagation which may significantly affect software maintenance costs [3. Therefore, detecting code clones has been an active area of research in the past two decades 4,5 .

Nowadays, popular question and answer (Q\&A) forums such as Stack Overflow 6] provide an immense amount of reusable code snippets. According to the Stack Overflow's statistics [7], about 50 million people around the world visit Stack Overflow website monthly, and around half of them are professional developers or university students. Stack Overflow provides rich information in the form of posts, each consisting of several text and code snippets, so software developers can get help on their software development problems by reusing readily available code snippets in their projects.

This study investigates how software developers reuse Stack Overflow's code snippets in the projects hosted on GitHub. GitHub [8] is a software repository hosting platform based on git, and it allows version control and collaboration on projects. There is a large number of references to the Stack Overflow discussions in the GitHub projects. Manes [9] has shown that open-source projects in GitHub reference Stack Overflow posts, and the average number of references to Stack Overflow is 176 per project. Code snippets from Stack Overflow referenced in the GitHub code make a great opportunity for us to study how developers reuse code snippets from Stack Overflow in their projects. Also, it can facilitate the studies about (i) detecting the types of code clones that exist between Stack Overflow and GitHub code snippets, (ii) the distribution of clone types between these two platforms, and (iii) predicting the types of code clones based on the reused code snippets on social coding platforms. In this work, we leverage clone detection techniques to study developers' code reuse 
practices. Based on the results of this work, we are able to: (1) identify which existing tools are able to detect clone types between Stack Overflow and GitHub code snippets, (2) offer insights into the clone types presented in the code snippets reused from Stack Overflow in GitHub projects, and (3) develop and evaluate multi-label classifiers in predicting the types of code clones.

\subsection{Motivation}

Stack Overflow, with 15 million users, 21 million questions, and 32 million answers, is one of the most popular Q\&A websites in the field of programming 1 . On the Stack Overflow website, programmers can ask questions and suggest solutions to the programming problems. These questions and answers contain two kinds of information blocks: (i) the text block is either the description of an issue or the solution to it, and (ii) the code block is a code snippet as a part of the answer to the asked question. Sometimes code snippets that are included in the answers are not directly authored on Stack Overflow, and instead, they are copied from other resources [10]. Reusing these code snippets in other projects can lead to license violations, bug propagation, reuse of outdated codes, or creation of vulnerabilities [10]. The main motivation behind this work is to explore how developers reuse code snippets from Stack Overflow in their GitHub projects - do they really copy-paste them entirely or do they adopt these code fragments to their own code bases by making necessary modifications?

By considering the reuse of code snippets from Stack Overflow as code cloning, we are able to study the adoption of these code snippets in other projects in terms of clone types. Code clones are divided to four different categories based on the degree of similarity between code pairs, which are Type I, Type II, Type III, and Type IV [11]. Although there are some studies on the quality of code snippets from online

\footnotetext{
${ }^{1}$ Date as 18 June 2021 from https://stackexchange.com/sites
} 
resources [12 and the frequency with which code snippets are reused from Stack Overflow in the GitHub projects [13, 14, there is no study focusing on the types of code clones between Stack Overflow and GitHub. Baltes et al. [15] have published a dataset called SOTorrent based on the Stack Overflow's data dump [16. SOTorrent provides access to all the contents of the Stack Overflow website, their version history, and files on other platforms like GitHub referencing any of these contents. Thanks to SOTorrent, we extend the dataset provided by Manes [9] in order to create correlative code pairs on Stack Overflow and GitHub.

The first part of this thesis focuses on the detection of code clones between Stack Overflow and GitHub code snippets. We try to discover the level of accuracy of existing tools in detecting the types of code clones between these two platforms and the challenges associated with using these tools. The second part of the thesis focuses on the prediction of clone types by using multi-label classification models. In a software system, around $5 \%$ to $50 \%$ of codebase contains clones, and maintenance is the most expensive phase of the software development cycle [11]. Code clones can be consider as repeated patterns in a software system. Because clone types represent the level of adoption that occurred in a cloned code, we will be able to better plan maintenance operations and development resources by predicting clones. Thus, we will be able to reduce the maintenance costs that arise as a result of cloning. The outcome of this work can provide fresh insights into the reuse of online code resources in software development. Moreover, the findings of this work can help in gaining a better understanding and contributing to the existing body of knowledge on code cloning in the context of empirical software engineering. 


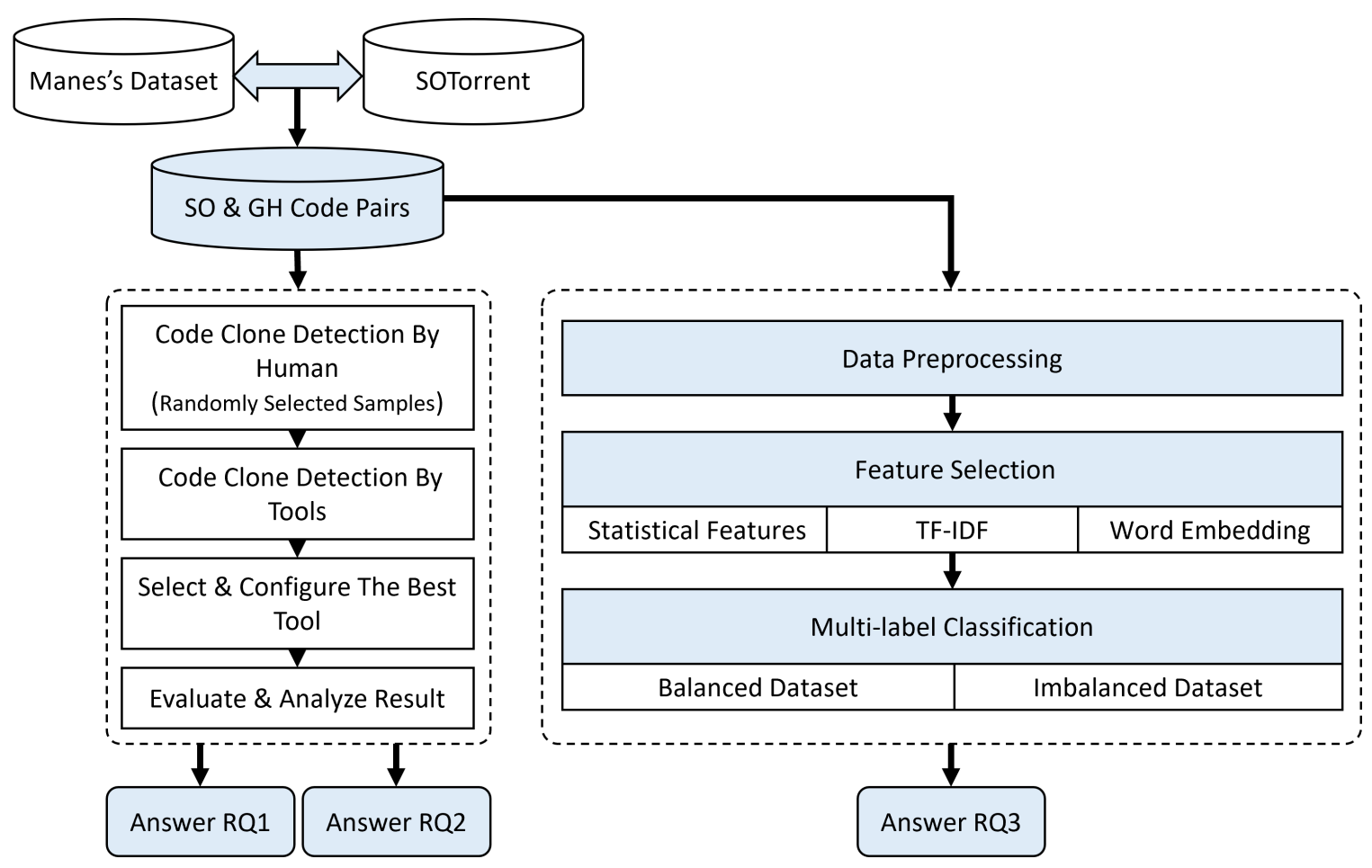

Figure 1.1: Our research roadmap.

\subsection{Research Questions}

The goal of this thesis is to gain better understanding and new insights into how knowledge, in the form of code snippets, available on Stack Overflow website is shared and reused in other software development projects. Figure 1.1 illustrates the roadmap we followed to answer the following research questions:

- RQ1: How accurately can clone detection tools identify code clones between Stack Overflow and GitHub?

In a general context, code clones are similar fragments of code; however, there is no agreement on this definition [17]. For instance, with regard to the degree of similarities or the size of code fragments, one expert may consider the fragments similar enough to be a code clone, while another expert may claim they 
are so disparate to be accounted a clone. Thus, detecting code clones is challenging even for human beings. This research question is critical in this study as it contributes the first step of detecting clone types. We try to find a tool capable of accurately detecting clone types in the four programming languages represented in the dataset. This research question involves both quantitative and qualitative research methods. The approach adopted here can help enhance our understanding of clone detection and its challenges associated with the multi-language code-snippet level.

- RQ2: What types of clones are present in the code snippets reused from Stack Overflow in the GitHub projects?

This research question builds up the next step that elaborates the previous findings in the RQ1 by analyzing the presence of different clone types between Stack Overflow and GitHub snippets. In addition, the clone size, efficacy of the programming language, complexity of the code snippet, etc. are quantified in this research question.

- RQ3: How accurately can we predict the clone type given two code snippets?

In order to tackle the last research question, the study moves on from understanding the distribution of clone types over two platforms to predicting clone types based on the prevalence of each type on these social coding platforms. This research question can yield an invaluable insight about the best way of extracting features from code snippets, what classification models can be utilized to predict clone types over two platforms, and how accurately we are able to predict clone types. 


\subsection{Contributions}

The major contributions of this research work are as follows:

1. Conducting a fine-grain analysis to understand how developers reuse code snippets from Stack Overflow in their own projects.

2. Evaluation of clone detection tools to detect clone types among Stack Overflow and GitHub code snippets.

3. Development and evaluation of machine learning models to predict clone types between two platforms' snippets.

4. Empirical evidence of code snippets reuse from Stack Overflow in GitHub projects.

5. A list of recommendations to the software developers, researchers and online programming platforms in supporting code reuse.

\subsection{Organization of the Thesis}

The structure of this thesis is as follows: Chapter 2 presents background and related research work relevant to our study. Chapter 3 describes the methodology, dataset, and data analysis techniques used in this work. Afterwards, the results and answers to the research questions are reported in Chapter 4 followed by the discussion on implications of this work in Chapter 5. Finally, the thesis concludes with Chapter 6 providing a summary of this study and future work directions. 


\section{Chapter 2}

\section{Background and Related Work}

This chapter lays out the foundational information required for this study and summarizes related work in this field.

\subsection{Code Reuse}

Code or software reuse is a common practice in software development. In the context of software engineering literature, code reuse has been called by many names like cutand-paste or copy-and-modify reuse [18], code scavenging [19], ad-hoc reuse [20], and opportunistic reuse 21. In recent years, with the emergence of online Q\&A websites such as Stack Overflow or online collaborative coding platforms such as GitHub, code reuse happens not only between two different versions of a software but also across different codebases since developers can access millions of open source projects.

Krueger [19] stated that reuse of code happens in three steps of selecting an entity, adopting, and integrating it in the target system. For a high level categorization, we divide software reuse into white-box and black-box approaches [22]. In the white-box, developers are permitted to change the internal operation of a reusable artifact, while in the black-box, they are not allowed to do so. Although, the black-box approach for software reuse seems less flexible, developers do not need to know how an artifact 
works. In contrast, the white-box approach enables developers to change an artifact based on their needs with the additional effort to understand and change it.

Most of the software reuse research emphasizes the design of software in a reusable fashion such as object-oriented programming [23] or component-based approaches 24 . 25]. Parsons and Saunders [26 mention that developers are able to perform tasks by anchoring their understanding to existing code and adjusting it based on their needs. This study was a promotion of a white-box reuse paradigm.

In addition to the white-box and block-box approaches, code cloning can be a simple way of reusing code. Code cloning refers to copied program code from a source which is used with or without modification. Although this is considered a bad practice [27], most developers prefer to do so due to the availability of today's online resources. Hence, similar to other approaches of code reuse, code cloning is a mechanism for the reduction of development time, the increase of developers' productivity, and the decrease of defect density [28,29].

Code cloning has both advantages and disadvantages. Since writing new code is an expensive activity and needs considerable time and effort, developers frequently search for code snippets on the web or other resources to be able to write code quickly and easily. However, some researchers believe that code clones may cause software maintenance to become more complicated [27]. For example, in the case of an emerging bug in a reused code snippet, all cloned instances need to be checked for the same bug, or alternatively, the modifications of the original code must be applied to the duplicated code.

There is no universally accepted definition for code cloning in the literature. In the Webster's dictionary [30], a clone is defined as "to make a copy of", while code cloning does not have a clear definition. In general, it refers to the approach of reusing existing code. Because it is difficult to distinguish which code fragment is original and which is cloned, all code fragments that are identical or similar to each other are 
referred to as code clones [11. Baxter et al. 31] define a clone as a program fragment that is identical to another fragment, and, a near miss clone as a fragment which is nearly identical to another. Other researchers use "similar code", "duplicated code" or "exact copy or a mutant of another function" 27,32,33. All these different definitions of code cloning result in vagueness which makes the clone detection process hard.

Between two code fragments, there are two types of similarity. Two code fragments can be textually similar or comparable based on their program text. This kind of clones are often the result of copying a code fragment from a location and pasting it to another location. In another similarity type, two code fragments are functionality similar. In this case, there is no textual similarity between two code fragments, and the functionalities or semantic of the two code fragments are the same [11].

Based on these two kinds of similarities, four code clone types have been defined 27, 31, 34, 35]. Table 2.1 illustrates the different code clone types and their definitions.

These types of clones define the degree of change from Type I to Type IV. Also, the table shows the analytical difficulty of detecting such clones rises from Type I to Type IV, with Type IV being the most difficult one. Even with extensive prior knowledge of the program's architecture and software design, detecting Type IV clones is not a trivial task. Whether the process is automated or not, the level of analytical complexity increases from Type I to Type IV.

\subsection{Code Clone Types}

In this section, we describe each clone code type and offer an example. 


\begin{tabular}{|c|c|c|}
\hline Similarity & Clone Type & Definition \\
\hline \multirow{3}{*}{ Textual } & Type I & $\begin{array}{l}\text { Identical code fragments but may have some vari- } \\
\text { ations in white-space, layout, and comments. }\end{array}$ \\
\hline & Type II & $\begin{array}{l}\text { Syntactically equivalent fragments with some } \\
\text { variations in identifiers, literals, types, white- } \\
\text { space, layout, and comments. }\end{array}$ \\
\hline & Type III & $\begin{array}{l}\text { Syntactically similar code with inserted, deleted, } \\
\text { or updated statements. }\end{array}$ \\
\hline Semantic & Type IV & $\begin{array}{l}\text { Semantically equivalent, but syntactically differ- } \\
\text { ent code. }\end{array}$ \\
\hline
\end{tabular}

Table 2.1: Code clone types and their definitions.

\subsubsection{Type I Code Clones}

In Type I clones, also called exact clones, two code fragments are identical. There may be some differences in white space (blanks, new lines, tabs), comments, and/or layouts. Two code fragments of Type I clones are shown in Table 2.2. After deleting the white space and comments, we can observe that these two fragments are textually similar and are considered as Type I code clones even though they have different layouts.

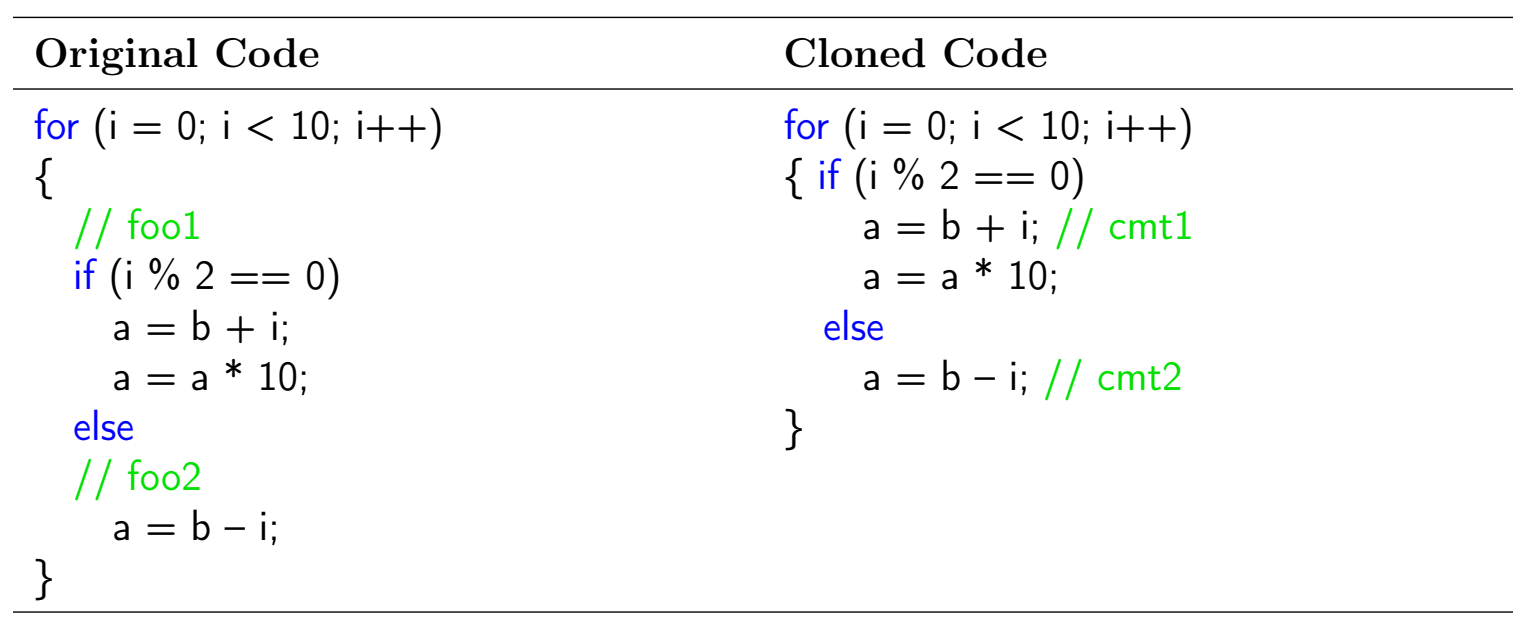

Table 2.2: An example of Type I code clone. 


\subsubsection{Type II Code Clones}

If there are some probable variations in user-defined identifiers such as variable names, methods, classes, and so on, two code fragments are considered Type II clones. But, the sentence structure and reserve words are essentially the same. An example of Type II clones is shown in Table 2.3. Although the layout, variable names, and value assignments of the two code fragments differ significantly, both fragments have a similar syntactic structure. Sometimes, Type II clones are also called renamed clones or parameterized clones [11].

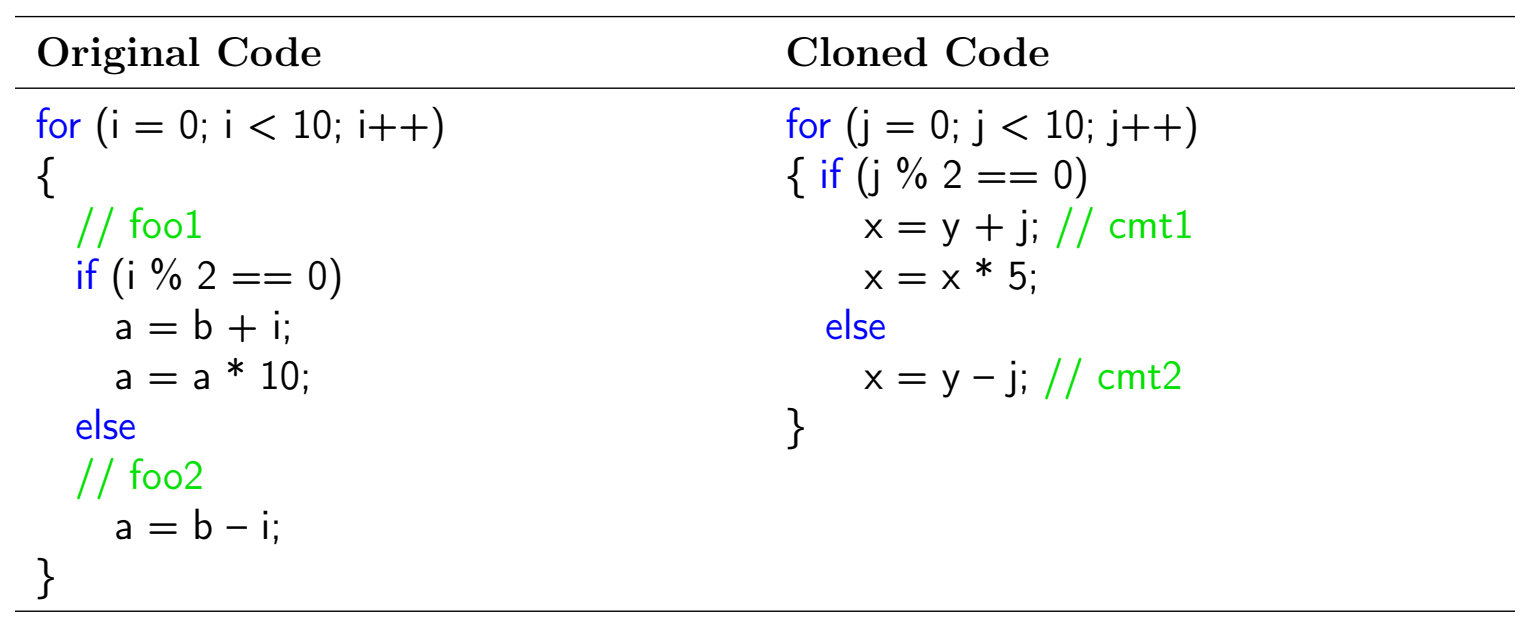

Table 2.3: An example of Type II code clone.

\subsubsection{Type III Code Clones}

The duplicated fragment is further adopted in Type III clones, with the statement(s) modified, added, and/or deleted. As we can see in Table 2.4, the copied code is extended by two statements $e=$ True and $a=2 * b$. Also, the $a=a * 10$ statement is deleted. This copied fragment could be a Type II code clone if it did not have these amended statements, but these changes in addition to the modified identifier name, make it a Type III clone or near-miss clone. 


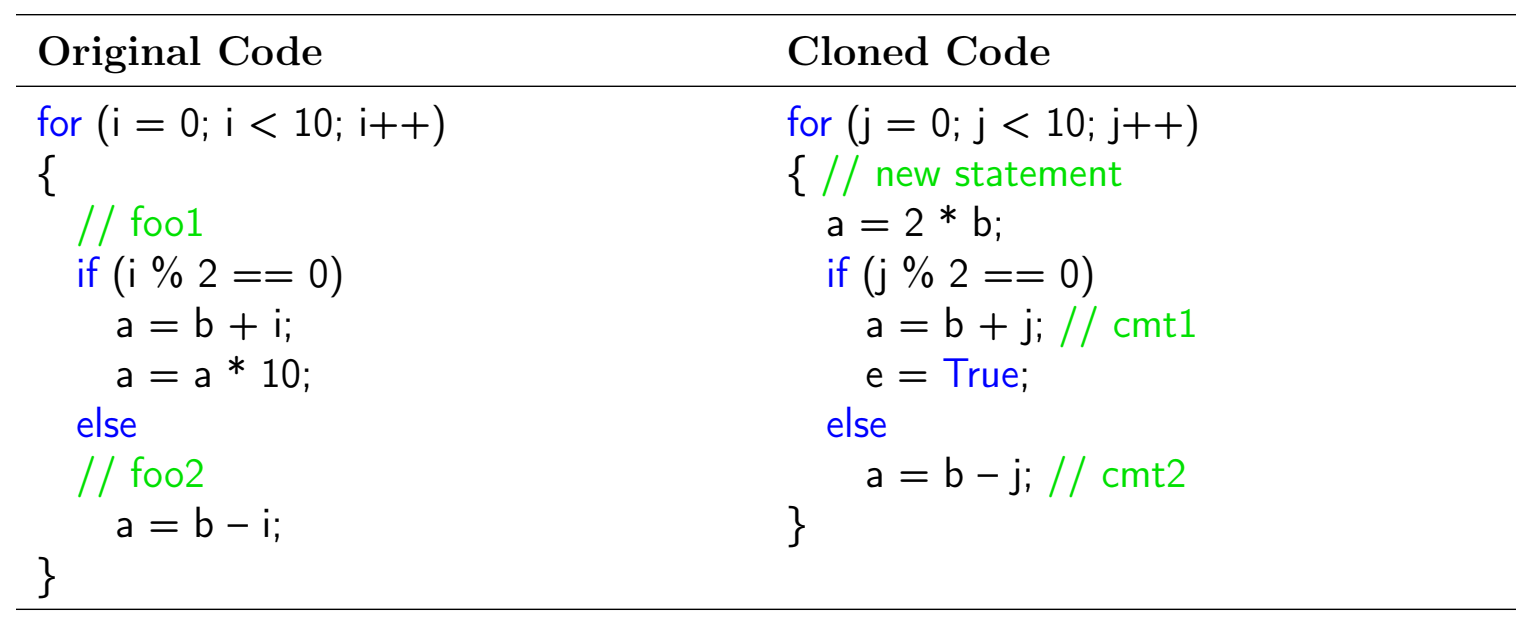

Table 2.4: An example of Type III code clone.

\subsubsection{Type IV Code Clones}

In Type IV clones or semantic clones, there is semantic similarity between code fragments. In other words, two different code fragments have the same logic and equivalent functionality. The degree to which the components behave similarly is measured by functional similarity. Table 2.5 presents two code fragments which are semantically equal. The cloned code is not necessarily copied from the original one, but it was written by two distinct developers who used the same rationale.

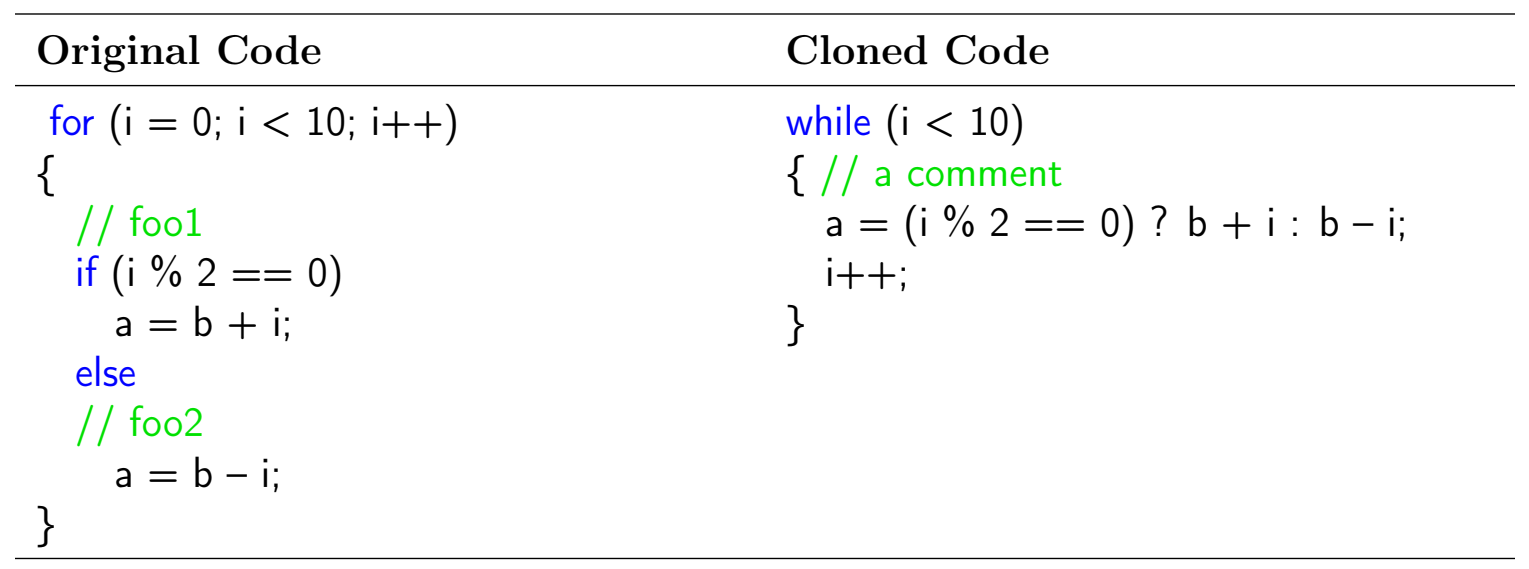

Table 2.5: An example of Type IV code clone. 


\subsection{Code Clone Detection Techniques}

A variety of tools and techniques for detecting code clones, as well as several comparisons and evaluation studies have been introduced so far. Clone detection has become critical in many software engineering topics such as aspect mining, program comprehension, plagiarism detection, copyright infringement investigation, code compaction, software evolution analysis, code quality analysis, bug detection and virus detection, extracting similar or synthetic similar code fragments is a need [36].

Each clone detection approach has its own set of characteristics. This set can be created by answering questions like 11$]$ :

- What kind of transformation/normalization does it apply to a code?

- What is the level of granularity?

- What comparison algorithm does it use?

- How much the computational complexity is?

- What kind of clones can be detected?

- Is it a language independent technique?

- Is output in the form of clone pair or clone class?

Code clone detection is primarily a two-phase process. In the first phase, the source code is converted to a format which allows more efficient comparison techniques to be used. The true matches are discovered during the comparison process in the second phase. Clone detection approaches are generally classified as follows [36]:

- Text-based: Consider the source code as a string or a series of lines. Two code fragments are compared against each other to find the sequences of the same lines. When at least two code fragments are discovered to be similar, they 
are returned as clone classes or clone pair using a detection approach. In the text-based approaches, no or little change on the source code is applied. The text-based approaches are able to detect Type I clones very well [37]. However, they can detect Type II and Type III clones as well [38].

- Token/Lexical-based: These approaches consist of two steps, lexical analysis and clone detection. In the lexical analysis step, the source code is transformed into a sequence of tokens with the help of laxer or parser. The token sequence is inspected for duplicate token subsequences, and then the original code fragments representing the duplicate subsequences are returned as clones [36. Tokenbased approaches are able to detect Type II clones efficiently [37, while they are also able to detect Type I and Type III clones [38].

- Tree-based: In tree-based clone detection techniques, with the help of a laxer or parser, the source code is parsed into a parse tree or abstract syntax tree. Following that, a tree matching approach is used to find related subtrees. The related source code of similar subtrees is returned as clone class or clone pair when it matches [36]. Tree-based approaches are able to detect all kind of clone types, specifically Type III clones 37, 38.

- Metric-based: In these approaches, metrics are used to measure clones in the software after they have been calculated from the source code. Statement metrics are calculated for syntactic units such as function software, or class, and then the values of these metrics are compared. A clone pair is formed when two syntactic units have the identical metric value. Like tree-based approaches, metric-based approaches are able to detect all kind of clone types [36 38].

- Semantic approach: The source code is represented as a program dependency graph (PDG) in these approaches. Because they analyze the semantic 
information of the source code, they are able to go one step further to get high abstraction of source code representation than others. Control flow and data flow information, which contain semantic information, are carried through the PDG. After obtaining a set of PDGs, the isomorphic subgraph matching technique is used to locate related subgraphs, which are then returned as clones [36. Although semantic approaches are used to detect Type IV clones, they can detect other types of clones as well [36, 37].

- Hybrid approach: A hybrid strategy is one that combines two or more clone detection approaches (textual, lexical, syntactic, or semantic). The hybrid method yields better results than the traditional method [36].

We now report on some code clone detection tools and techniques used in earlier studies. Table 2.6 summarizes the studies organized by year of publication. In the table, we have concentrated on the most relevant characteristics of these tools and techniques for our own study. According to the previous works, code clone detection has been a research area of interest for software engineers over the past decade. Although in recent years researchers have attempted to exploit the benefits of machine learning in the domain of code clone detection, for effective code clone detection, the current knowledge still needs to be expanded.

Kamiya et al. [39 provided a clone detection tool called CCFinder. CCFinder is based on transformation rules and a token-based comparison, as well as performance and efficiency optimization techniques. The authors have also given measures for selecting intriguing clones, such as length, clone population, and radius. After that, other versions of CCFinder like CCFinderX [58] and CCFinderSW [59] are introduced. CCFinderX is a newly updated and developed version of CCFinder. Its new design and technologies are aimed at boosting performance, allowing user-side 


\begin{tabular}{|c|c|c|c|c|c|c|}
\hline $\begin{array}{l}\text { Author }(s) / \\
\text { Year }\end{array}$ & Name & $\begin{array}{l}\text { Classic/ML } \\
\text { App. }\end{array}$ & Approach & Level & $\begin{array}{l}\text { Able to De- } \\
\text { tect Clone }\end{array}$ & Language \\
\hline $\begin{array}{l}\text { Kamiya et al. } \\
(2002) \text { 39 }\end{array}$ & CCFinder & Classic & Token-based & $\begin{array}{l}\text { No Limita- } \\
\text { tion }\end{array}$ & Type I, II, III & Multiple \\
\hline $\begin{array}{l}\text { Basit and } \\
\text { Jarzabek } \\
(2005) \quad 40\end{array}$ & Clone Miner & ML-based & $\begin{array}{lc}\text { Token } & \text { Ex- } \\
\text { traction } & + \\
\text { FIM } & \end{array}$ & Class or File & Type I, II & Java \\
\hline $\begin{array}{l}\mathrm{Li} \text { et al. } \\
(2006)\end{array}$ & CP-Miner & ML-based & $\begin{array}{l}\text { Token Ex- } \\
\text { traction }+ \\
\text { Sequential } \\
\text { Pattern } \\
\text { Mining }\end{array}$ & $\begin{array}{l}\text { No Limita- } \\
\text { tion }\end{array}$ & Type I, II & $\mathrm{C}, \mathrm{C}++$ \\
\hline $\begin{array}{ll}\text { Jiang } & \text { et al. } \\
(2007) & 42\end{array}$ & DECKARD & ML-based & $\begin{array}{l}\text { Tree Parsing } \\
+ \text { Clustering }\end{array}$ & Method & $\mathrm{N} / \mathrm{A}$ & Multiple \\
\hline $\begin{array}{l}\text { Roy and } \\
\text { Cordy } \\
(2008) \text { 43 }\end{array}$ & NiCad & Classic & $\begin{array}{l}\text { Text-based } \\
\text { hybrid }\end{array}$ & Function & Type I, II, III & Multiple \\
\hline $\begin{array}{l}\text { Gode and } \\
\text { Koshke } \\
(2009) \quad 44\end{array}$ & ICLONES & Classic & Token-based & $\begin{array}{l}\text { No Limita- } \\
\text { tion }\end{array}$ & Type I, II, III & Multiple \\
\hline $\begin{array}{l}\text { Uddin et al. } \\
(2011)\end{array}$ & SimCad & ML-based & $\begin{array}{l}\text { Hash Finger- } \\
\text { print + DB- } \\
\text { SCAN Algo- } \\
\text { rithm }\end{array}$ & $\begin{array}{l}\text { Function or } \\
\text { Block }\end{array}$ & Type I, II, III & Java, C \\
\hline $\begin{array}{l}\text { Koschke } \\
(2014) \quad 46\end{array}$ & $\begin{array}{l}\text { Suffix-Tree- } \\
\text { Based Inter- } \\
\text { System } \\
\text { Clone Detec- } \\
\text { tion }\end{array}$ & ML-based & $\begin{array}{l}\text { Suffix Tree }+ \\
\text { Decision Tree }\end{array}$ & File & Type I, II & Java \\
\hline $\begin{array}{l}\text { Kodhai and } \\
\text { Kanmani } \\
(2014) 47\end{array}$ & CLoneManager & Classic & $\begin{array}{l}\text { Light Weight } \\
\text { Hybrid Ap- } \\
\text { proach }\end{array}$ & Method & $\begin{array}{l}\text { Type I, II, } \\
\text { III, IV }\end{array}$ & Java, C \\
\hline $\begin{array}{l}\text { White et al. } \\
(2016) 48\end{array}$ & $\begin{array}{l}\text { Learning- } \\
\text { Based Ap- } \\
\text { proach }\end{array}$ & ML-based & $\mathrm{AST}+\mathrm{RNN}$ & Method & $\begin{array}{l}\text { Type I, II, } \\
\text { III, IV }\end{array}$ & Java \\
\hline $\begin{array}{l}\text { Sajnani et al. } \\
(2016) 49\end{array}$ & SourcererCC & Classic & Token-based & Method & Type I, II, III & Java, C, C\# \\
\hline $\begin{array}{l}\mathrm{Li} \text { et al. } \\
(2017) 50\end{array}$ & CCLearner & ML-based & $\begin{array}{l}\text { Token Ex- } \\
\text { traction } \\
+\quad \text { Deep } \\
\text { Learning }\end{array}$ & Method & Type I, II, III & Java \\
\hline $\begin{array}{l}\text { Wei and Li } \\
(2017) 51\end{array}$ & $\mathrm{CDLH}$ & ML-based & $\begin{array}{l}\text { AST-based } \\
\text { LSTM + } \\
\text { Hash Finger- } \\
\text { print }\end{array}$ & Method & $\begin{array}{l}\text { Type I, II, } \\
\text { III, IV }\end{array}$ & Java, C \\
\hline $\begin{array}{l}\text { Kim et al. } \\
(2017) \quad 52\end{array}$ & VUDDY & Classic & Text-based & Method & Type I, II & $\mathrm{C}, \mathrm{C}++$ \\
\hline $\begin{array}{l}\text { Saini et al. } \\
(2018)\end{array}$ & Oreo & ML-based & $\begin{array}{l}\text { Calculate } \\
\text { Metrics } \\
\text { Siamese } \\
\text { DNN }\end{array}$ & Method & Type I, II, III & Java \\
\hline $\begin{array}{l}\text { Zhao and } \\
\text { Huang } \\
(2018) \quad 54 \\
\end{array}$ & DeepSim & ML-based & $\begin{array}{l}\text { CFG \& DFG } \\
+ \text { FFNN }\end{array}$ & Method & $\begin{array}{l}\text { Type I, II, } \\
\text { III, IV }\end{array}$ & Java \\
\hline $\begin{array}{l}\mathrm{Yu} \text { et al. } \\
(2019) 55\end{array}$ & TBCCD & ML-based & $\begin{array}{l}\text { AST + Tree- } \\
\text { based CNN }\end{array}$ & Method & Type IV & Java \\
\hline $\begin{array}{l}\text { Nafi et al. } \\
(2019) 56\end{array}$ & CLCDSA & ML-based & $\mathrm{AST}+\mathrm{DNN}$ & Method & $\mathrm{N} / \mathrm{A}$ & $\begin{array}{l}\text { Java, } \\
\text { Python }\end{array}$ \\
\hline $\begin{array}{l}\text { Zhang et al. } \\
(2019) \quad 57\end{array}$ & ASTNN & ML-based & $\begin{array}{l}\mathrm{AST} \\
\mathrm{BiRNN}\end{array}$ & Method & $\begin{array}{l}\text { Type I, II, } \\
\text { III, IV }\end{array}$ & Java, C \\
\hline
\end{tabular}

Table 2.6: Code clone detection techniques and tools. 
preprocessor customisation, and giving interactive metrics analysis. CCFinder is language independent and can detect clones in a variety of programming languages. It can also detect clones of Type I, II, and III.

Clone Miner [40] is a tool for detecting simple clone types in the level of classes and files. It employs ANTLR to convert the source code into tokens, and then use the suffix array algorithm to detect simple clones. Using frequent itemset mining techniques, a consistent pattern of clone classes in distinct files is discovered. This is accomplished by identifying common clone patterns. The clustering technique uses two metrics to group files with similar clone patterns: file percentage coverage and file token coverage. Clone Miner is tested on Java programs to detect Type I, II clones.

Li et al. 41] have introduced the CP-Miner tool. It uses closed sequential pattern mining which is a type of frequent sub-sequence mining used by CP-Miner. To locate common subsequences and prune false-positive subsequences, the algorithm performs a depth-first search. CP-Miner locates clones without modifying the source code. CP-Miner are able to work with $\mathrm{C}$ and $\mathrm{C}++$ code to detect Type I, II clones.

DECKARD [42] is another machine learning based tool for clone detection. It is a language-independent tool since the parser generator is built using the syntax grammar of source code. Through the parser, the source code is transformed into a parse tree. For subtree and subtree forest, vector generations are conducted. A comparable vector is clustered to identify clones using locality sensitive hashing. Clustering vector and the generic vector grouping techniques are used to detect size-sensitive clones.

Roy and Cordy [43] have introduced NiCad. NiCad is a text-based hybrid clone detection tool. This method uses a two-stage approach: first, identify and normalize prospective clones using flexible pretty-printing and code normalization, then compare potential clones using simple text-line comparisons utilizing dynamic clustering. NiCad is able to detect Type I, II, and III clones. It was designed to detect clones in the functions written in $\mathrm{C}$, but after developing the TXL grammar, it can now be 
used with a wider range of programming languages.

ICLONES [44] is a token-based detector as well. It is an incremental clone detection technique that detects clones over numerous software versions. The results of a revision's analysis are reused in the next revision's analysis. This eliminates the need to re-analyze source code that has not changed between revisions. ICLONES has also mapped clones of successive revisions based on the modifications to the generalized suffix tree. IClONES is able to detect Type I, II, and III code clones for multiple programming languages.

SimCad 45 is a tool developed by Uddin et al. to detect near-miss clones. The program accepts bits of source code and normalizes them. For each code snippet, the Simhash algorithm is utilized to build a hash fingerprint. The data generated by the preceding technique is handled using two-level indexing. The DBSCAN technique, which estimates Hamming distance between hash fingerprint values, is used to group similar code fragments into a cluster.

Koschke 46] has proposed an inter-system clone identification approach based on suffix trees. For clone detection, the approach transforms the subject system into a suffix tree. The suffix tree is compared with files in the corpus to find similar files between the subject system and the corpus. In addition, the hashing approach is recommended for boosting the speed of comparison. If the hash values of two files, one in the subject system and the other in the corpus, are not the same, there is no need to compare the file and suffix tree.

With the computation of metrics combined with a simple textual analysis strategy, Kodhai et al. [47] have suggested a lightweight hybrid approach to detect methodlevel clones for both textual and functional similarity types. CloneManager, the name of their tool, can detect all four types of clone in $\mathrm{C}$ and Java projects.

White et al. 48 have presented a technique based on learning. It is divided into two tiers. The lexical level implements the recurrent neural network to turn fragment 
into embeddings. The syntactic level entails converting an abstract syntax tree to a binary tree, which is subsequently converted to an olive tree. A comparison between nodes is performed using the greedy technique. A recursive neural network can find a match using either embedding or tree nodes. In terms of evaluation, they sampled and manually evaluated 398 file- and 480 method-level pairs across eight real-world Java systems; $93 \%$ of the both samples were evaluated to be true positives in all all four clone types.

SourcererCC is a token-based accurate near-miss clone detection tool introduced by Sajnani et al. [49]. SourcererCC is able to achieve large-scale clone detection on a normal workstation by using an optimized partial index and filtering heuristics. Moreover, it is capable of detecting Type I, II, and III clones in Java, C, and C\# methods.

CCLearner [50] is a token-based clone detection procedure. In the feature extraction procedure, tokens are taken out from both clone and non-clone method pairs. Then, tokens are categorized in the eight groups. CCLearner generates a vector for all tokens existing in the method. A sim score is used to measure the similarity of vectors. A deep neural network binary classifier is utilized in the training step to train procedures for clone and non-clone method pairs. During the testing stage, method pairs are extracted after loading the source code into the procedure, and binary classifiers identify clone and non-clone method pairs.

Wei and Li [51] have developed the CDLH (Clone Detection with Learning to Hash) framework for detecting a functional clone. There are two distinct phases. To obtain representations for all code snippets, code snippets are converted into an abstract syntax tree (AST) and subsequently into long short term memory (LSTM) during the extraction phase. The former phase's representations are converted into hash codes during the hashing phase. A Hamming distance is used to determine code snippets that are close to each other. CDLH could detect all types of clones in both 
the Java and $\mathrm{C}$ languages used in the experiment.

VUDDY [52] is a technique for detecting vulnerable clones. VUDDY's modeling technique is divided into two stages: 1) pre-processing and 2) clone detection. Its design principles focus on length filtering while retaining accuracy and extending scalability through functional-level granularity. So that allowing it to discover vulnerable clones from the rapidly growing pool of open source software. VUDDY can detect Type I and II clones and supports source code written in $\mathrm{C}$ and $\mathrm{C}++$.

Saini et al. 53 have proposed an Oreo for clone detection. Oreo focused on detecting clones in the Twilight Zone. When considering clone types as a spectrum, the Twilight Zone is located between Type III and Type IV, where clones, while still exhibiting some syntactic similarities, are extremely difficult to detect. The first step in the Oreo detection pipeline is metrics calculation from given source codes. The query and candidate method portions are generated by input partitioning, and for each candidate approach, an inverted index is produced. For each query method, the index displays a group of candidate ways. When the metric hash of methods is compared, Type I, II clones occur if they are equivalent. If they are not equal, Oreo creates a pair of candidate methods and build feature vectors. Feature vectors are then fed into a Siamese neural network to determine the presence of Type III, IV clones in pairs. Oreo was tested on BigCloneChange which consists of clone and non-clone pairs of Java language methods.

DeepSim [54] is another technique introduced by Zhao and Huang. It is divided into two main stages. During the first stage which is the semantic representation stage, the source code is turned into a control and data flow graph. The feature matrix is formed by encoding these graphs. The neural network extracts significant properties and reveals them in latent representation during the code similarity measure stage. Classifiers used this latent representation to determine coding similarity. Deepsim is implemented for Java at the method level and is able to detect all types of clone. 
Yu et al. [55] offered a tree-based convolution (TBCCD) approach for clone detection. In this approach, the cosine similarity between code snippets is checked to see if they are Type IV clones or not. At first, the code is transformed into an abstract syntax tree and subsequently into embedding using the convolution layer. The fully connected layer is utilized after pooling to determine the vector value of code fragments for cosine similarity.

The CLCDSA approach [56] (Cross-Language Code Clone Detection Using Syntactical Features and API Documentation) is presented by Nafi et al. The language of source code is first recognized. Then, an abstract syntax tree of source code is used to extract features. After that, numeric metrics are computed for all features. Cosine similarity is determined from the numeric metric value of two code fragments to determine if they are clones or not. The metrics are immediately incorporated into a model for learning and subsequently predicting the label of an unlabeled pair using deep neural networks on a dataset consists of Java, C\#, and Python programming contests.

For clone detection, Zhang et al. [57] introduce ASTNN (AST-based neural network) approach. In this approach, a large abstract syntax tree is split into smaller trees, and then a recurrent neural network $(\mathrm{RNN})$ is utilized to generate vector representations of the larger tree without affecting source code line order. This vector representation is employed in the both detection and classification of source code clones.

\subsection{Classification Problem}

Classification is a supervised predictive modelling task in machine learning where a class target/label is predicted for a given sample of input data [60]. In other words, a classification predictive model estimates a mapping function from input variables to 
discrete output variables. In the training phase, to learn how provided input variables relate to the target class, a classifier uses some training data with many examples of inputs and outputs. Classification problems can be divided into three categories based on the target classes:

1. Single label classification. It entails assigning a target label to an input sample. This classification can be further subdivided as follows:

(a) Binary classification. It refers to classification problems that have two class labels. In most binary classification problems, one class represents the normal state and the other represents the aberrant state. The normal state class is assigned the class label 0, while the abnormal state class is allocated the class label 1. Some algorithms, such as Logistic Regression [61 and Support Vector Machines [62], are built primarily for binary classification and do not support more than two classes by default. This type of classification could be applied to a variety of situations, including but not limited to spam detection [63], disease diagnosis [64, and quality control 65.

(b) Multi-class classification. Classification problem with more than two class labels are referred to as multi-class classification. Character recognition 66 and face classification 67 are two examples of real-world challenges that require this form of classification. Although many real-world problems are designated as multi-label classification, binary classification methods can be extended to solve multi-class problems. This requires fitting multiple binary classification models for each class versus all other classes (known as one-vs-rest) or a single model for each pair of classes (called one-vs-one).

2. Multi-label classification. Classification tasks with two or more class labels, 
where one or more class labels can be predicted for each sample, are referred to as multi-label classification. This differs from binary and multi-class classification, which predict a single class label for each sample. Multi-label classification algorithms cannot be utilized directly with binary or multi-class classification techniques. For that, several machine learning algorithms have been developed, which can be broadly classified into three categories: 1) Problem Transformation Methods, 2) Algorithm Adaption Methods, and 3) Ensemble Methods. Section 3.3.5 goes over these methods in detail. This classification is continually evolving as the number of real-world applications grows [68]. Its application in classifying movies is a common example; the same film could be classified as both an action and a comedy at the same time 69.

3. Multi-output/task classification. The purpose of multi-output classification is to learn a classification rule whose output is a set of labels, or vector. Solving a multi-output problem with machine learning entails creating a predictive model that outputs a set of (two or more) labels that measure different concepts at the same time. It means two or more separate (but related) classification problems are solved concurrently within the same model. A multi-output classification problem is practically equivalent to numerous simultaneous (multi-tasked) single-label classification problems, which highlights the fact that a multi-output classification problem is multitask-classification. Text categorization, image generation, and visual question answering |70 are some of the applications of this classification type.

\subsection{Mining Software Repositories}

The amount of data associated with software projects is growing, which results in developers and maintainers becoming overwhelmed. Mining software repositories 
(MSR) research area allows researchers to acquire a better understanding of continually changing artefacts tied with long-term projects. Reusing components or maintaining existing software systems account for the majority of the cost of software projects, rather than new developments. As a result, project history knowledge or patterns are extremely beneficial for software evolution. One of the goals of the MSR, as Thomas Zimmermann said, is to "learn from past achievements and failures to help developers produce better software" 71 .

Data mining is defined as "the process of automatically discovering useful information in large data repositories |72]", and MSR is described as "a field which analyzes the rich data available in software repositories to uncover interesting and actionable information about software systems and projects [73]". MSR has a similar definition to data mining, but data mining is a broader field than MSR. In contrast to data mining, which analyzes quantitative, nominal, or text data, MSR analysis necessitates software domain knowledge because its sources are mainly code, bug reports, test suits, design documents, or other types of development artifacts 71 . Without software engineering domain knowledge, extracting and processing this data is difficult, and cannot be understood just through statistics.

In MSR, problems like how developers make the most use of code, finding and predicting bugs are the more visited ones. The mining of software repositories to discover the top utilized APIs has been described by Moritz et al. 74. Hsu and Lin 75 have also scanned software sources for code snippets that can be reused. Chang and Blei [76] used topic modelling to exploit this knowledge for debugging and traditional bug finding. Similarly, such data can be used to search for earlier problem fixes in code histories [77], which can be complemented by bug repositories like Bugzilla [78]. Versioned software contracts extracted from software repositories can be a useful tool for understanding and supporting evolution. Yan et al. [79 have attempted to extract software evolution from Git repositories. They constructed 
contracts for each method implemented in different versions of source code, then tracked how these contacts evolved over time.

This work is connected to MSR since we mine huge repositories such as Stack Overflow and GitHub, extract specific data, construct new dataset for research, analyze the extracted data, and present our findings.

\subsection{Code Reuse in Mining Software Repositories}

Code reuse is one of the topics covered by the MSR field of research. Code reuse is defined as "the use of existing software or software expertise to develop new software" [80]. Various researchers have attempted to build different strategies on how to reuse existing code as code reuse from in-house projects or online forums has become an attractive problem $5,80,82$. Furthermore, some studies on code reuse from online forums or repositories such as Stack Overflow or GitHub are conducted [13,73, 80,83. The prior work in this field is summarised in Table 2.7. As we can see, this is a novel topic of research that has attracted the interest of researchers in the past five years.

According to the Stack Overflow licence, the original question and answer must be referenced in the target code, as well as the adoption of a compatible licence by the derived work. Baltes et al. [84 have reported the findings of an empirical study comparing referenced and non-referenced usages of Stack Overflow code snippets in GitHub's Java projects. They have employed the PMD Copy-Paste Detector, which is a token-based code clone detector, to locate non-referenced usages of three different sets of Stack Overflow code snippets in a random sample of prominent GitHub Java projects. Also, they have built regular expressions that matched the code snippets from the ten most popular Java responses on Stack Overflow. They have used these regular expressions in BigQuery to discover non-referenced usages in all Java projects in their data set. After that, they have manually analyzed random samples of Stack 


\begin{tabular}{|c|c|c|c|}
\hline Author(s)/Year & Objective & Approach & Dataset \\
\hline Baltes et al. (2017) 84 & $\begin{array}{l}\text { Examine how code snip- } \\
\text { pets from SO are used and } \\
\text { attributed in GH projects. }\end{array}$ & $\begin{array}{l}\text { 1) Create dataset, 2) } \\
\text { PMD Copy-Paste De- } \\
\text { tector to detect non- } \\
\text { referenced SO codes, } \\
\text { 3) Regular expressions } \\
\text { matching, 4) Manually } \\
\text { analyzed random samples. }\end{array}$ & $\begin{array}{l}\text { GH, Stack Exchange } \\
\text { APIs, GHTorrent, and } \\
\text { SO }\end{array}$ \\
\hline Yang et al. (2017) 13 & $\begin{array}{l}\text { Look into how much the } \\
\text { SO snippets are used in } \\
\text { GH projects. }\end{array}$ & $\begin{array}{l}\text { 1) Extract Python func- } \\
\text { tion from GH \& code snip- } \\
\text { pets from SO, 2) Cross ref- } \\
\text { erenced SO snippets with } \\
\text { GH functions based on } \\
\text { three measures of similar- } \\
\text { ity, 3) Analyze the results. }\end{array}$ & $\begin{array}{l}909 \mathrm{k} \text { non-forked Python } \\
\text { projects in GH, and Stack } \\
\text { Exchange }\end{array}$ \\
\hline $\begin{array}{l}\text { Abdalkareem et al. } \\
(2017) 85\end{array}$ & $\begin{array}{l}\text { The impact of code reuse } \\
\text { from SO in the context of } \\
\text { mobile apps. }\end{array}$ & $\begin{array}{l}\text { 1) Extract Java code form } \\
\text { SO and } 22 \text { open source } \\
\text { Android apps, 2) Mea- } \\
\text { sure code similarity with } \\
\text { CCFinder tool, 3) Identify } \\
\text { reused code, } 4 \text { Analyze the } \\
\text { results. }\end{array}$ & $\begin{array}{l}22 \text { Open source Android } \\
\text { apps from F-Droid, and } \\
\text { SO data dump (published } \\
\text { March } 16,2015 \text { ) }\end{array}$ \\
\hline $\begin{array}{lll}\text { Lotter et al. (2018) } & 80 \\
\end{array}$ & $\begin{array}{l}\text { How the use of online } \\
\text { Q\&A forums affects fu- } \\
\text { ture software maintenance } \\
\text { and the correct use of li- } \\
\text { cences to avoid legal con- } \\
\text { cerns. }\end{array}$ & $\begin{array}{l}\text { 1) Extract java code from } \\
\text { SO, SourceForge, and GH, } \\
\text { 2) Find clones with the } \\
\text { help of CCFinderX clone } \\
\text { detection tool, 3) Analyze } \\
\text { the results. }\end{array}$ & $\begin{array}{l}\text { SO data dump, Source- } \\
\text { Forge, and GH }\end{array}$ \\
\hline Nishi et al. 201986 & $\begin{array}{l}\text { understand why develop- } \\
\text { ers copy code from SO to } \\
\text { the software development } \\
\text { tutorials website, and how } \\
\text { duplicated content evolves } \\
\text { over time. }\end{array}$ & $\begin{array}{l}\text { 1) Collecting data from } \\
\text { Android tutorial websites } \\
\& \text { SOTorrent 2) Find } \\
\text { clones by a code clone de- } \\
\text { tection tool. 3) Applied } \\
\text { a coarse grained filtering } \\
\text { to detect template snip- } \\
\text { pets to exclude. 4) Ana- } \\
\text { lyze the results. }\end{array}$ & $\begin{array}{l}599 \text { Android tutorials, and } \\
\text { SOTorrent }\end{array}$ \\
\hline Digkas et al. (2019) 83 & $\begin{array}{l}\text { Is there a difference be- } \\
\text { tween the quality of SO } \\
\text { code fragments and the } \\
\text { quality of the projects } \\
\text { where the code is reused? }\end{array}$ & $\begin{array}{l}\text { 1) Retrieved code snippets } \\
\text { from SO and selected GH } \\
\text { projects, 2) Matching SO } \\
\text { and GH code snippets by } \\
\text { looking for clones, } 3 \text { ) ana- } \\
\text { lyzing the results. }\end{array}$ & SO data dump, and GH \\
\hline $\begin{array}{l}\text { Ragkhitwetsagul et al. } \\
(2021) 10\end{array}$ & $\begin{array}{l}\text { Investigate how online } \\
\text { code clones occur, as well } \\
\text { as the potential conse- } \\
\text { quences of reusing them } \\
\text { in software systems. }\end{array}$ & $\begin{array}{l}\text { 1) Create dataset, 2) } \\
\text { Clone Identification with } \\
\text { Simian \& SourcererCC, 3) } \\
\text { Clone merging, 4) Vali- } \\
\text { dation \& classification, 5) } \\
\text { Analyze results. }\end{array}$ & $\mathrm{SO}$, and Qualitas corpus \\
\hline
\end{tabular}

Table 2.7: Code reuse in the MSR research field. 
Overflow references in Java files to see how developers refer to Stack Overflow in source code comments. According to their findings, at least one reference to Stack Overflow was found in $3.22 \%$ of all studied repositories and $7.33 \%$ of the popular ones. Furthermore, they have noticed that developers would rather refer to the entire Stack Overflow thread than a single answer.

Yang et al. [13] wanted to know how often code snippets from Stack Overflow make their way into open source projects. For this, they looked at Python programs and a collection of 909k non-forked Python projects posted on GitHub, as well as the Stack Exchange. They took all of Stack Overflow's multi-line Python code samples, processed all of GitHub's Python projects, and broke them down into functions. The Stack Overflow snippets were then compared to these functions using three different measures of similarity: exact match, token match, and near-duplication. With the use of SourcererCC tools, they discovered near-duplication similarities. They discovered that exact duplication at the function level was extremely infrequent (less than 1\%), whereas token-level duplication was more common. Around 1.1\% of GitHub blocks, on the other hand, were near-duplicated to Stack Overflow. For Stack Overflow blocks, which were used in GitHub, this ratio was roughly $2 \%$.

The utilisation of Stack Overflow code in mobile apps was investigated by Abdalkareem et al. [85]. They have compiled a dataset from Stack Overflow data dump and F-Droid 11 that included 22 Android open source applications. The Stack Overflow code snippets were all Java programs with at least 30 lines of code. CCFinder clone detection tools found reused code from Stack Overflow within mobile apps after the corpus was developed. The authors have looked at Type I and Type II clones. This work examined reuse in terms of both the percentage of Stack Overflow posts that were reused in mobile apps and the percentage of code in mobile apps that were reused in Stack Overflow in order to undertake analysis. They discovered that feature

\footnotetext{
${ }^{1}$ https://www.f-droid.org/
} 
additions and enhancements are the most common reasons for Stack Overflow code duplication in mobile apps.

Lotter et al. [80] have investigated the impact of online Q\&A forums on future software maintenance and legal challenges. They have looked into the degree of code reuse within Stack Overflow as well as between Stack Overflow and popular open source software like Sourceforge ${ }^{2}$ and GitHub. This study only concentrates on the Java programming language. They used CCFinderX with the default parameter settings to find repeated codes. The results showed that about $77.2 \%$ of project files contained clones from Stack Overflow. As a result, it advised that Java developers should be aware of licence difficulties and the risks associated with adhoc copying.

Nishi et al. 86 have conducted a study to understand why developers copy code from Stack Overflow to the software development tutorials website, and how duplicated content evolves over time. They gathered 2,504 Java code samples from 599 Android tutorials across five websites. Stack Overflow code snippets were also gathered from SOTorrent [1]. To determine clone codes, a scalable clone detection was applied to the code snippets. The study's findings revealed a set of categories for postings containing duplicated code blocks, as well as some of the most likely justifications for transferring code from tutorials to Stack Overflow.

Digkas et al. [83 have investigated the effect of reusing Stack Overflow code snippets on the target system's quality. They gathered Java code snippets from Stack Overflow and GitHub's data dumps. PMD 3 with the ability to detect copy/paste code is used to check for duplicates between the two collected corpora. After that, SonarQube calculated the Technical Debt (TD) density of code snippets. The findings of this study backed up Stack Overflow's strong developer reputation, suggesting that code reuse can increase software quality in addition to increasing efficiency.

\footnotetext{
${ }^{2}$ https://sourceforge.net/

${ }^{3}$ https://pmd.github.io
} 
"Online code clones", or code snippets transferred from software systems to online Q\&A websites, were introduced by Ragkhitwetsagul et al. [10]. The authors of this study examined how online code clones emerge and the potential consequences of reusing them in software systems. They mined Stack Overflow posts, detected online code clones, and analyzed the clones to reveal toxic code snippets (outdated code or license-violating code). Their findings revealed that certain codes posted on Stack Overflow from Qualitas were out of date, while others may have violated the licence of their original software.

\subsection{Source Code as Natural Language}

Programming languages are a bridge between human and computers. Because source codes communicate with both humans and computers, they are naturally bi-modal. The bi-modality of code causes it to have similarities and contrasts with text. Some researchers believe that, despite the fact that programming languages are complicated, versatile, and powerful in principle, and that the codes created with them are in an artificial language, they are a natural product of human efforts [87]. The term natural relates to the field of natural language processing (NLP), which aims to automatically process texts in natural languages such as English. Researchers have used NLP techniques to extract features [88], search code snippets [89], and forecast programming languages from source code [90].

NATURALIZE, a refactoring tool based on NLP, was introduced by Allamanis et al [91]. It is the first tool to discover identifiers that violate code standards and make ideas to improve stylistic consistency by learning local style from a source. NATURALIZE is a general tool that may be used to analyze any language that has a laxer and a parser, since token sequences and abstract syntax trees are employed.

Sachdev et al. [89 have suggested a tool called Neural Code Search (NCS) that 
could search through code snippets. With the help of the FastText model, they built a continuous vector embedding of each code fragment at method-level granularity. They also mapped the given query in a natural language to the same vector space. Then, they employed vector distance to simulate code fragment relevancy to a given query.

Using NLP and Machine Learning, Alreshedy et al. [90 have proposed a classifier to predict the programming language of questions posted to Stack Overflow. They used the TF-IDF model to extract three sets of features from: 1) the title and text body of each post, 2) code snippets, and 3) a mix of both. Their findings suggested that textual information is easier for a machine learning model to learn than information from code snippet.

In this work, we have applied code clone detection and MSR techniques to answer our research questions. Also, we have evaluated the use of NLP and machine learning techniques in predicting code clone types between Stack Overflow and GitHub snippets (as presented in Section 3.3.4). 


\section{Chapter 3}

\section{Methodology}

We have discussed studies conducted on Stack Overflow in Chapter 2, The majority of these studies were concerned with determining whether there are code snippets from Stack Overflow in other projects and, if so, what impact they have on quality or bug propagation. Manes and Baysal's study [2] revealed that GitHub developers do make use of Stack Overflow conversations in their code. In this study, we extended their dataset by creating a set of code pairs from Stack Overflow and GitHub in order to investigate the amount of adoption developers applied to use Stack Overflow snippets in their GitHub projects. Furthermore, we want to see if we can predict clone types.

This chapter consists of three main parts: Section 3.1 explains the concept used to built dataset for further analysis by mining Stack Overflow. Section 3.2 describes the overall methodology of code clone detection, while Section 3.3 presents our methodology for predicting code clone types based on multi-label classification technique.

\subsection{Datasets}

In this study, we have used SOTorrent [1] to extend GHCodeSnippetHistory dataset [2], in order to determine to what degree code snippets from Stack Overflow are used in GitHub projects. 
SOTorrent [15]. SOTorrent is a public dataset derived from the official Stack Overflow data dump [16] and the Google's BigQuery GitHub dataset [92]. It provides access to the version history of Stack Overflow posts and referred Stack Overflow posts to external resources. In SOTorrent, a discussion thread is made up of a series of posts. A question, a comment, or an answer to a question can all be included in a discussion thread. Depending on how the authors prepare the content of a post, each post may have text and code blocks. The textual portion of posts that are questions, answers, or descriptions to a question is kept in text blocks. Code blocks are pieces of code that explain a problem or a solution. In SOTorrent, version histories of changes are preserved for each of these blocks.

Beyond the version history of content, SOTorrent links Stack Overflow posts to external resources in two ways: 1) by extracting external URLs from the post text, and 2) by providing a table of links to Stack Overflow posts located in the source code of GitHub projects [15]. In the SOTorrent's schema, this table is called PostReferenceGH. This connection is crucial for our research since it allows us to map Stack Overflow posts to GitHub projects and identify all Stack Overflow snippets used in GitHub projects. Two further tables in the SOTorrent schema were used to extract the code blocks from the Stack Overflow post. These tables are PostHistory and PostBlockVersions. PostHistory keeps track of the type and date of all the changes made to each post, while PostBlockVersion keeps track of all the changes made to both text and code blocks [1]. The SOTorrent dataset schema is depicted in Figure 3.1. We referred each code block from Stack Overflow that is referenced in GitHub as reused code snippet.

GHCodeSnippetHistory [2]. Manes and Baysal [2] have mined SOTorrent and GHTorrent $[93]$ to produce a dataset called GHCodeSnippetHistory. The goal was finding an insight about the evolution of code snippets on both platforms. There are 22,900 projects in their final dataset, 33,765 Stack Overflow references mapped 


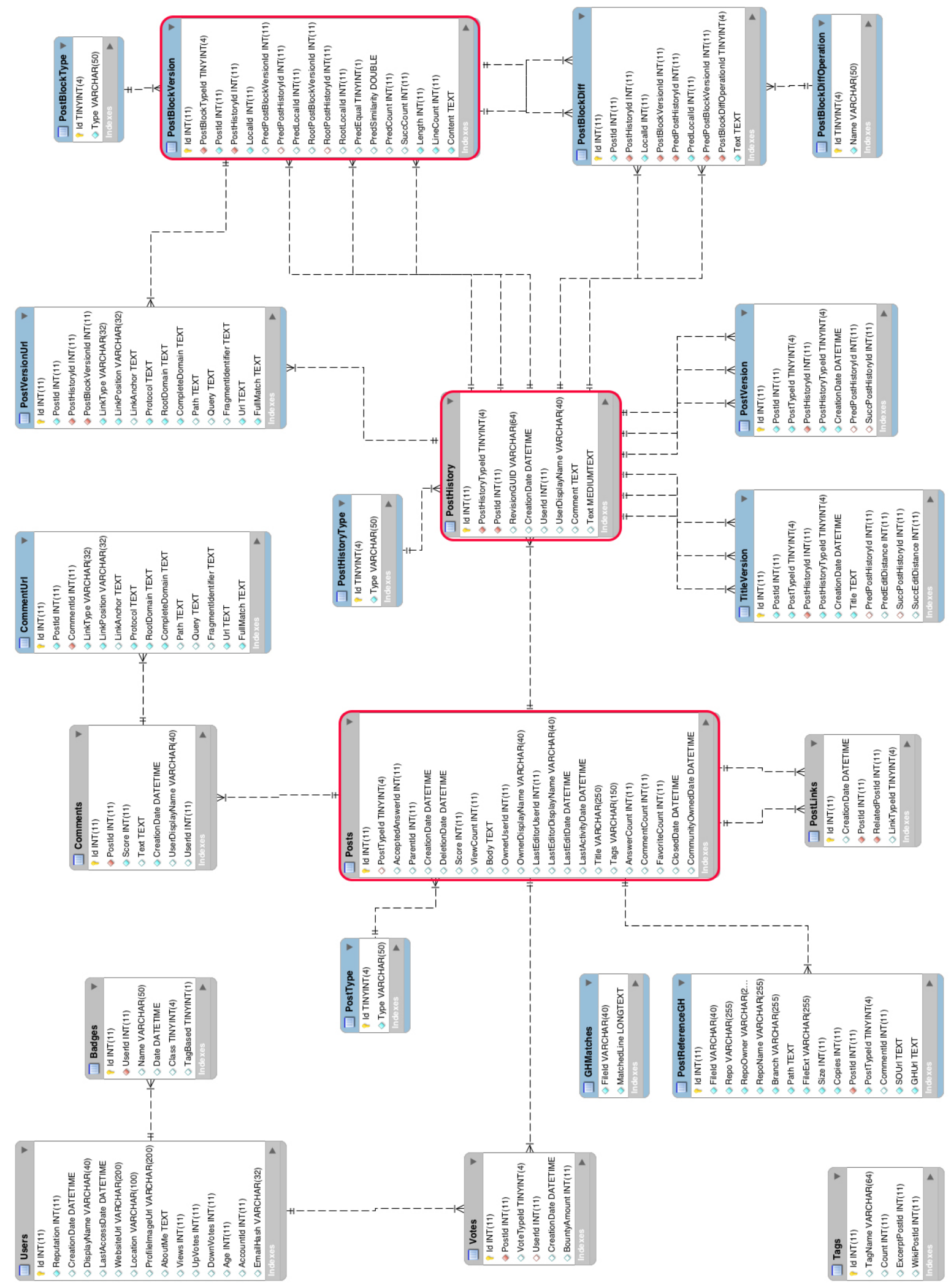

Figure 3.1: SOTorrent dataset layout [1]. 


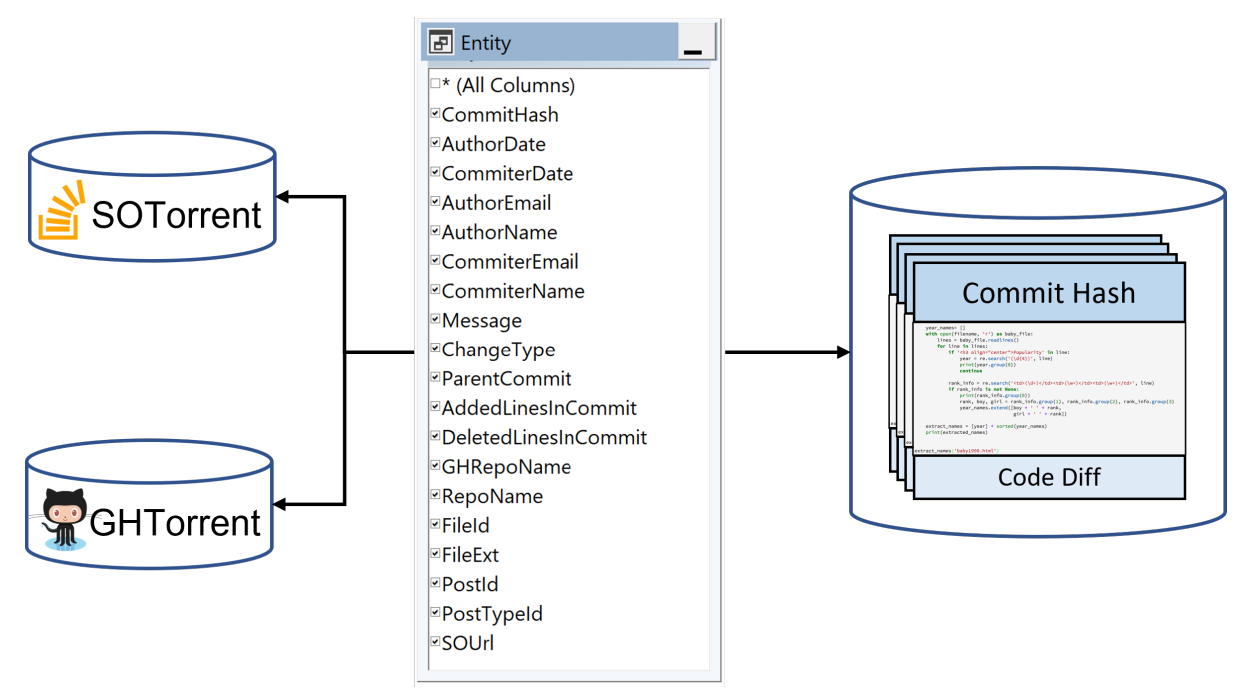

Figure 3.2: GHCodeSnippetHistory dataset layout [2].

to 4,634 Stack Overflow posts, and a total of 71,274 GitHub commits for four programming languages Java, JavaScript, PHP, Python. Figure 3.2 illustrates the GHCodeSnippetHistory dataset schema. We augmented this dataset by generating code pairs from Stack Overflow code snippets and the GitHub commits that corresponded to them.

Creating a Dataset. In our study, we required Stack Overflow code snippets that are referenced in the GitHub projects. The GHCodeSnippetHistory [2] dataset contains GitHub commits with Stack Overflow post references. We have extracted correlated Stack Overflow code snippets for those GitHub commits from SOTorrent with the help of BigQuery [92]. Then, we mapped those commits to the Stack Overflow post version based on their created date. The process of creating Stack Overflow and GitHub code pairs is depicted in Figure 3.4 .

GHCodeSnippetHistory keeps the relation with GitHub commits and Stack Overflow posts. First, all Stack Overflow "postIds" were taken from the GHCodeSnippetHistory dataset. As GHCodeSnippetHistory used SOTorrent version 2019/06/21, we used the same version dataset to prevent any ID conflict. Some extracted "postIds" belonged to a question post, while some of them did not contain code blocks. We did 


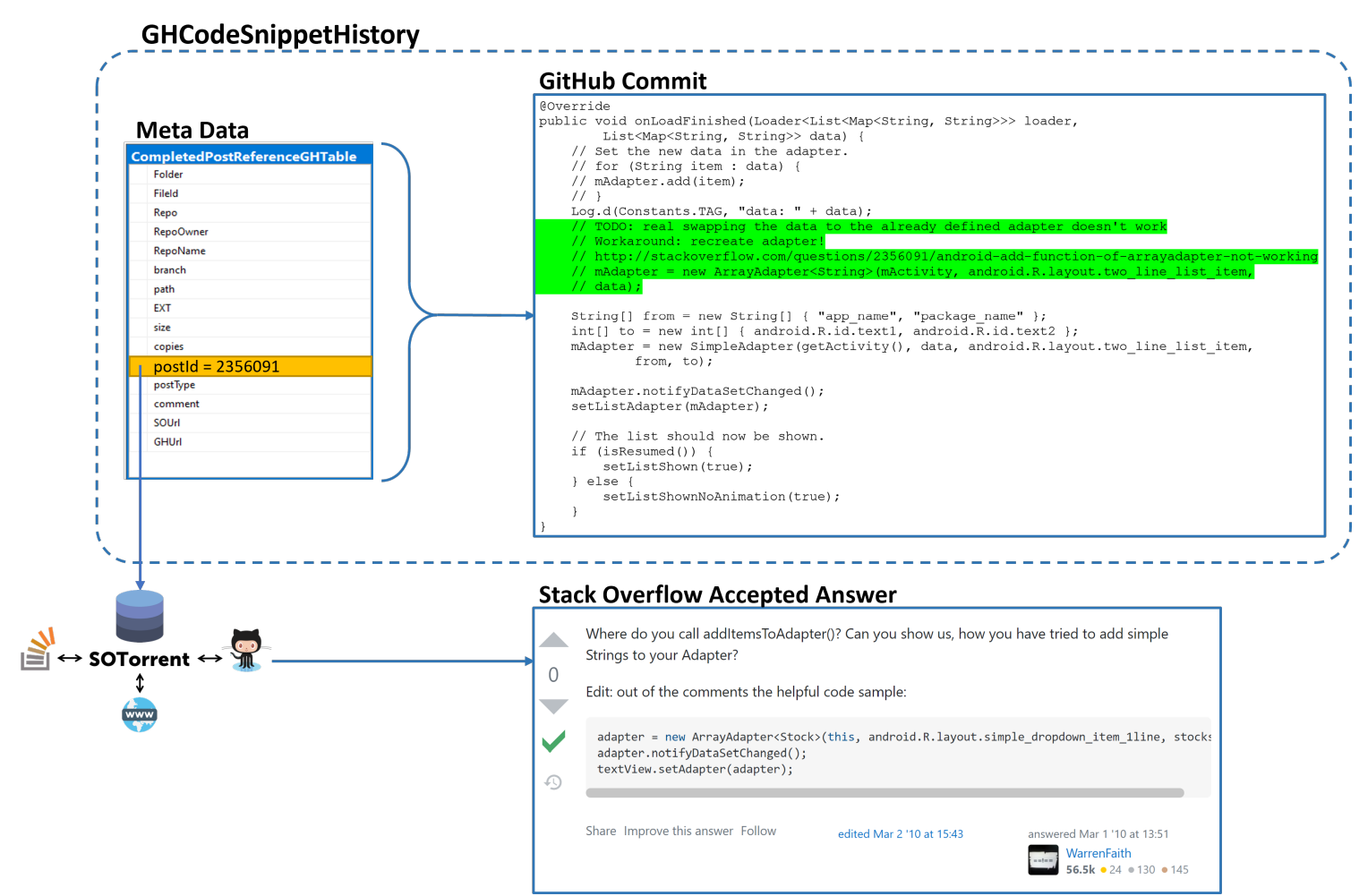

Figure 3.3: Data collection: adding a Stack Overflow snippet to the GHCodeSnippetHistory dataset.

not consider a commit if it was linked to a Stack Overflow post that did not contain a code block. In addition, some commits referenced question posts. We replaced them with accepted answers or top voted answer (if no accepted answer is available) in their discussion thread, as they are the ones that are more likely to be reused. Figure 3.3 illustrates an example of collecting snippets from Stack Overflow to expand the GHCodeSnippetHistory dataset. At the end of this step, our dataset contained 55,076 commits with reference to a Stack Overflow post that contains at least one code block. Because some posts have several code blocks, the total amount of the Stack Overflow code blocks is 106,484 .

Each post may have several code or text blocks, as seen in Figure 3.1. Furthermore, there are several versions of each block which show the evolution of one block over time. There is no version mapping between Stack Overflow and GitHub. There 
are two fields in GHCodeSnippetHistory and SOTorrent dataset called "AuthorDate" and "CreationDate", respectively. "AuthorDate" is used to determine when a commit was made. The "CreationDate" property indicates when a Stack Overflow block was modified. With the help of these fields, we were able to map all GitHub commits in the GHCodeSnippetHistory dataset to a related code block of a Stack Overflow post. We mapped a commit to the first Stack Overflow code block when its "AuthorDate" was greater than "CreationDate". Finally, our dataset consists of a total of 61,253 code pairs from Java, JavaScript, PHP, and Python. Table 3.1 illustrates the number of code pairs in our dataset for each programming language. We have randomly selected 48 code pairs from each programming language, 192 pairs in total, to verify whether our mapping was correct. Using the Stack Overflow post URL specified in the GitHub commit, we checked if code blocks were correctly extracted and mapped. We also used these samples for various assessments in the other steps.

As a final step, we applied a data cleanup process on the remaining GitHub code snippets. GHCodeSnippetHistory dataset was created to study code evolution. Thus, each sample in this dataset, as shown in Figure 3.5, has added and deleted rows indicated by "+" and "-" marks. We need to delete removed lines from the GitHub commits since they may contain code clones that no longer exist, or further adoption has been applied to them, and the clone type has changed in the current commit. Hence, we applied a cleanup process over all GitHub commits in our dataset, and removed any lines annotated with "-".

The prepared dataset consists of two SQL tables and a main folder containing code pairs placed in sub-folders. Each sub-folder has two files: one for Stack Overflow code snippets and one for GitHub. The codePairsMetaData database stores information about code pairs such as ID, language, filenames, post and post history ID in SOTorrent, and so on. In our dataset, each code pair has its own ID. We can locate code pairs in the directories using their Id. In addition, the CodePairsFeature table stores 


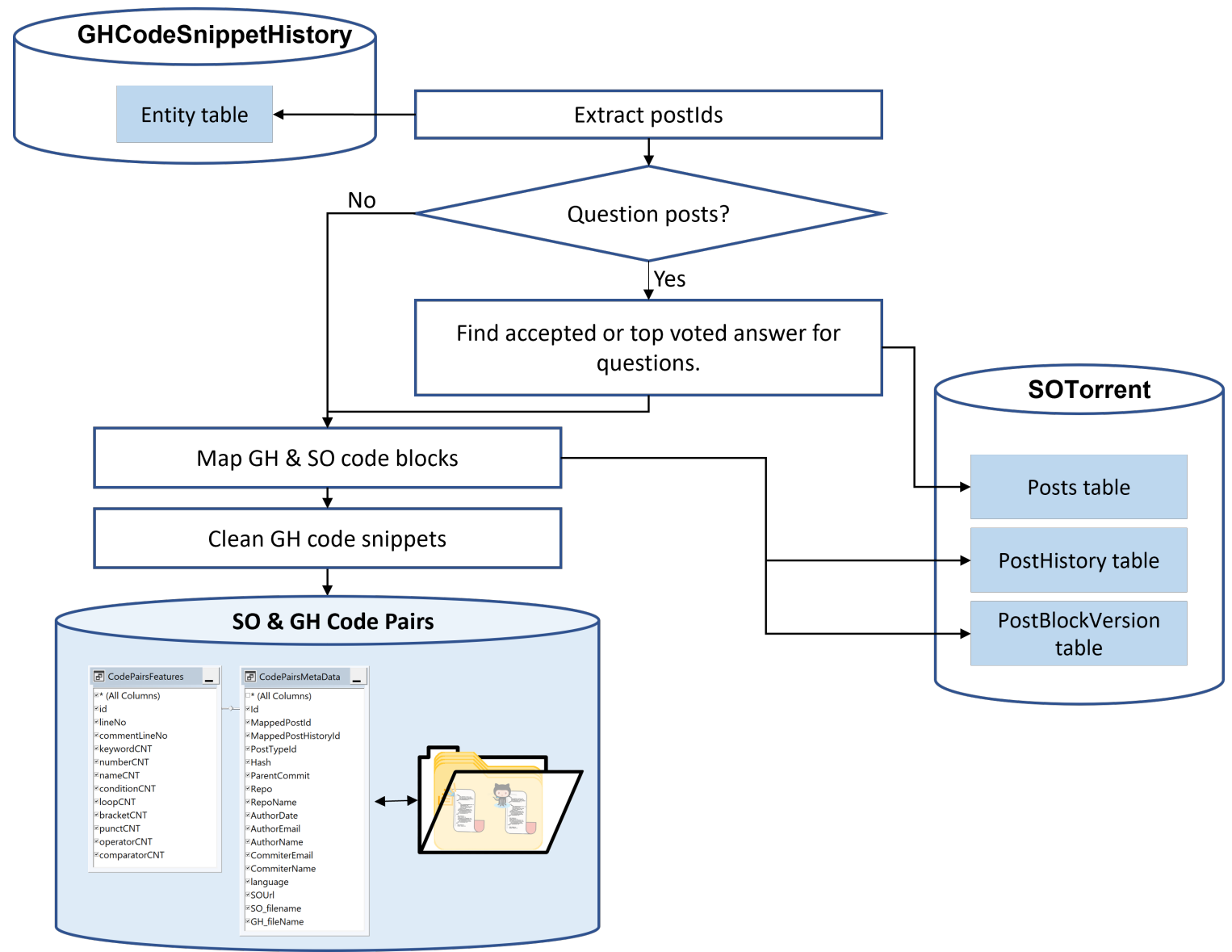

Figure 3.4: The workflow of creating our dataset.

additional information about each code pair, such as the number of lines, comments, keywords, and so on. We present them in more detail in Section 3.3.4.1.

\begin{tabular}{ll}
\hline Language & Number of Code Pairs \\
\hline Java & 4,266 \\
\hline Javascript & 43,435 \\
\hline Python & 2,724 \\
\hline PHP & 10,828 \\
\hline Total & $\mathbf{6 1 , 2 5 3}$ \\
\hline
\end{tabular}

Table 3.1: The number of code pairs for each programming language in the dataset.

Table 3.2 shows the number of characters in terms of the minimum, maximum, 


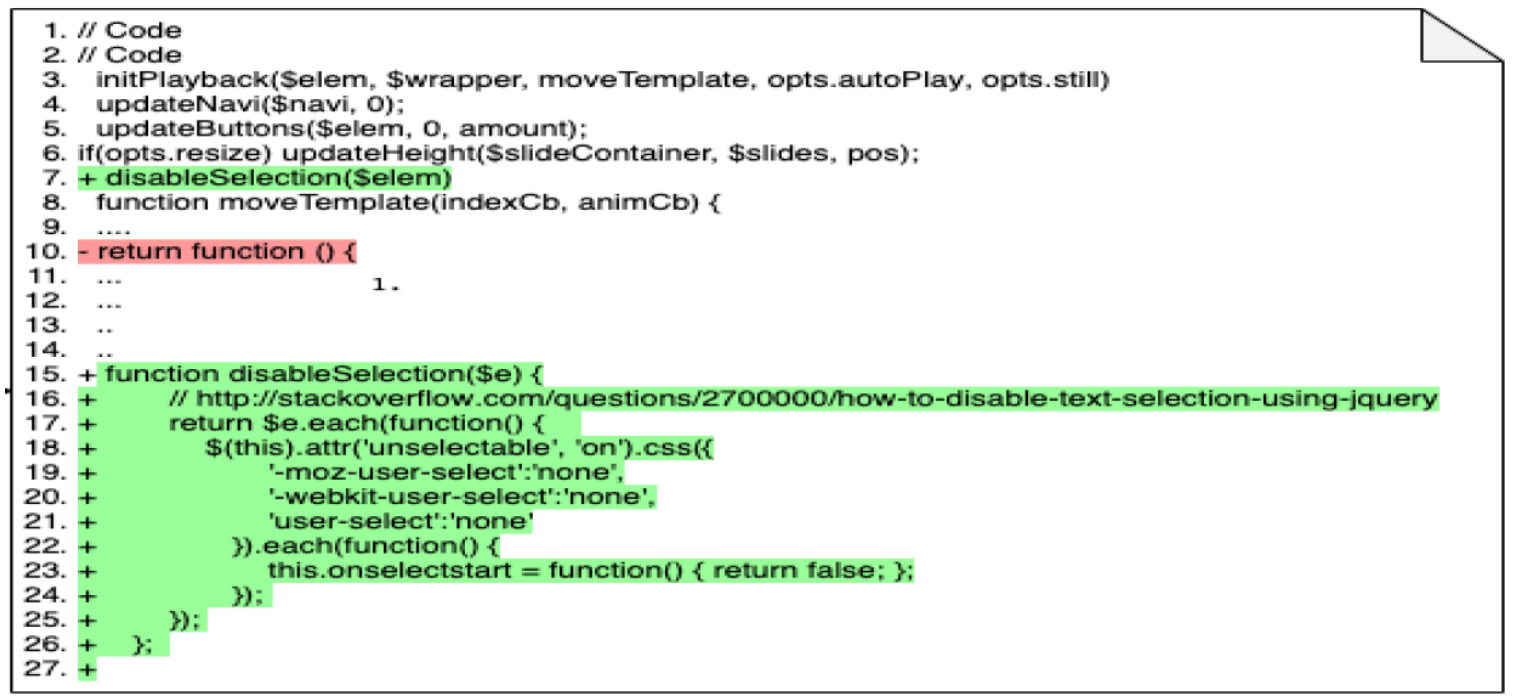

Figure 3.5: A sample commit [2].

average, and standard deviation of code snippets for each programming language. JavaScript has the longest code snippets among GitHub samples with the average of 23,262 characters, whereas Java has the shortest with the average of 1,073 charterers. However, standard deviation is considerable in all programming languages, particularly in JavaScript, indicating a wide range of lengths. In the Stack Overflow samples, Java has the longest and PHP has the shortest code snippets with the average of 267 and 183 characters, respectively. Generally, GitHub code snippets are longer than Stack Overflow code snippets in our dataset.

\subsection{Code Clone Detection}

One of the goals of this thesis is to determine if existing clone detection tools can detect code clones between the Stack Overflow and GitHub code snippets. We test four state-of-the-art clone detection tools on a subset of 192 samples from our dataset. Meanwhile, these samples are analyzed by human subjects to validate the clone detection tools' outcome. After comparing the results of the human evaluations with 


\begin{tabular}{cccccc}
\hline Platform & Language & Min & Max & Avg & Std Dev \\
\hline \multirow{4}{*}{ GitHub } & Java & 27 & 20,950 & 1,073 & $\pm 1,549.4$ \\
\cline { 2 - 6 } & JavaScript & 30 & 243,625 & 23,262 & $\pm 48,443.2$ \\
\cline { 2 - 6 } & PHP & 38 & 94,795 & 5,509 & $\pm 9,743.4$ \\
\cline { 2 - 6 } & Python & 41 & 34,039 & 1,490 & $\pm 2,296.1$ \\
\hline \multirow{5}{*}{ Stack Overflow } & Java & 11 & 4,954 & 267 & \pm 668.2 \\
\cline { 2 - 6 } & JavaScript & 12 & 1,942 & 236 & \pm 267.2 \\
\cline { 2 - 6 } & PHP & 14 & 1,296 & 185 & \pm 242.6 \\
\cline { 2 - 6 } & Python & 13 & 2,053 & 203 & \pm 292.7 \\
\hline
\end{tabular}

Table 3.2: A general overview of code pairs in terms of character count.

the tools, the best performing tool, ICLONES, is selected. Then, we used IClones for detecting clone types across the entire dataset.

Figure 3.6 illustrates the process of clone detection in this thesis. We prepared a collection of code pairs and randomly selected 192 samples from it. Section 3.1 provides details about the dataset and selected samples. Section 3.2.1 describes the tools we have evaluated for detecting clones, while Section 3.2 .2 describes the process of detecting clones by human subjects. Section 3.2 .3 explains how we select and configure the best tool to run over our dataset. Finally, we manually assessed the detected clones by selecting 60 code pairs at random among the code pairs with clones. The details are provided in Section 3.2.3.4.

\subsubsection{Overview of the Tools Used}

The first step in this research is to identify code clone types in code snippet pairs collected from Stack Overflow and GitHub. There are various tools and strategies for code clone detection, as we covered in Section 2.3. However, there are a number of restrictions in choosing the clone detection tools in this study. The biggest limitation stems from the nature of Stack Overflow code snippets, which are mostly incomplete 


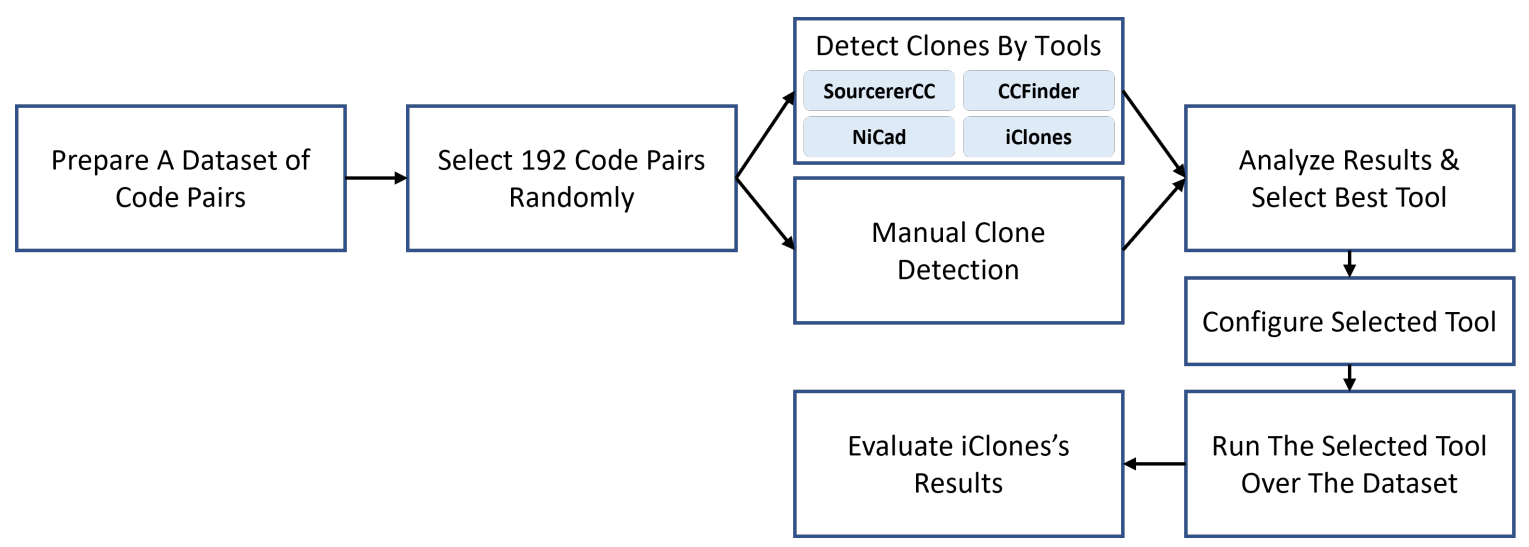

Figure 3.6: Clone detection process.

classes or methods. As a result, a detector must be able to handle code snippets that are not compilable or full blocks. Therefore, due to this constraint many tools based on Abstract Syntax Tree are not suitable for our work. Also, since the code snippets in our dataset cover four different programming languages, the tools must be able to parse them and detect clones. Furthermore, tools must be able to return the type of detected clones. The second limitation is important for two reasons. We require clone types to study what type of changes developers make when they reuse Stack Overflow code snippets in their GitHub projects. In addition, we must create labels our dataset in order to build prediction models. Hence, we have tested four clone detector tools over 192 randomly selected samples of the dataset. We described these samples in Section 3.1 .

We have tried four clone detectors including SourcererCC [49], CCFinder [39], NiCad [43], and ICLONES [44]. All of them are well-known clone detectors that have been used in previous studies. We run them over the 192 randomly selected samples in Section 3.1. While being aware of the tools' characteristics and limitations, we put them empirically to the test on a selected sample. SourcererCC, CCFinder, and ICLONES are token-based tools, whereas NiCad is a hybrid text-based tool. All of these tools are able to detect Type I, II, and III code clones. We tried to choose 
tools that could be used with a variety of programming languages. SourcererCC has limitations, as it only works with Java, C, and C\# program codes. However, since it is a token-based tool, we tried to test it for other programming languages as well. Tool that is able to recognise clones in a way that was more comparable to human assessment is chosen to detect clones across the entire dataset.

As SourcererCC was able to detect clones at either function or block level granularity, it was unable to parse our samples, with the exception of three code pairs. However, it did not return clone types for those three samples. NiCad is a hybrid text-based clone detector which is able to detect code clones for a wide range of programming languages. However, it can detect clones in the level of function. Thus, NiCad failed to parse selected code snippet pairs.

CCFinder and ICLONES are token-based clone detectors that can detect clones for many programming languages. Both of them were able to run on our samples. However, CCFinder did not return the type of detected clones. It was able to report the ratio of similarity in a code pair. ICLONES could report clone types. As a result, we chose ICLONES since it met all of our criteria.

\subsubsection{Manual Assessment of Code Clone Detection}

To evaluate the precision of clone detection tools, we required a baseline. Thus, 192 code pairs as described in Section 3.1 were distributed among 26 graduate and undergraduate volunteer students to manually identify code clones. Code pairs were broken into 13 groups. There were at least 15 code pairs in each group. Since detecting clone is not a trivial task even for human subjects [11], each group was evaluated separately by two participants. Prior to this study with participants, we have prepared and shared an instruction manual document describing the different clone types, their definitions and examples. We also offered further explanations to participants and answered their questions during the study. Each participant was 


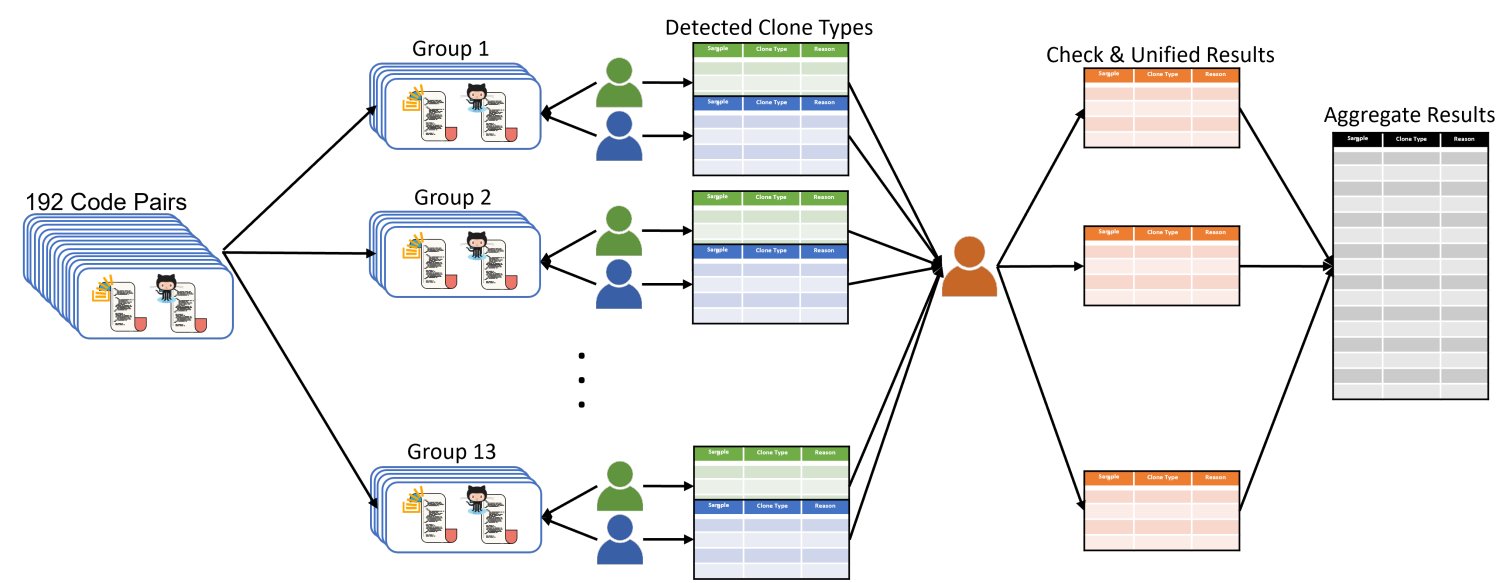

Figure 3.7: Manual clone detection process.

asked to indicate the type of any existing clone in a code pair, as well as to provide a justification for it. Finally, we compared the obtained results for each group. If two participants agreed on the clone type(s), we kept their answers as the result; otherwise, we checked the code pair to verify which participant's answer was correct. Finally, we assessed the agreement between the two coders using Cohen's Kappa coefficient 94 . Cohen's Kappa coefficient is a well-known statistical measure for determining the level of inter-rater agreement on categorical scales. The resulting co-efficient is scaled from -1.0 to +1.0 , with a negative value indicating worse than chance agreement, zero indicating exact chance agreement, and a positive number indicating greater than chance agreement. The process of manual clone detection and validation is illustrated in Figure 3.7. As the outcome of this manual process, we have produced 192 code pairs with known code clone types that can be used for the evaluation of tools. 


\subsubsection{Clone Detection Using IClONES}

ICLONES is an incremental clone detection tool created by Göde and Koschke [44. It extracts clone evolution data using a token sequence matching algorithm. Tokenbased tools depend on syntactic similarities and are able to detect Type I, II, and III clones, while Type IV clones rely on semantic similarities. Since ICLONES has a token-based nature, it is able to detect Type I, II, and III code clones. In this study, we employed ICLONES to detect clones between Stack Overflow and GitHub code pairs.

\subsubsection{Preparing the Source Code}

In this step, we must consider two points. First, the structure of the ICLONES' input. Second, the programming languages that it can support. Because ICLONES is intended to extract clone evolution data, it has a specific input format structure. However, it can execute on a single file. Users can select between "directory" and "single" input structures with the informat parameter. The "directory" format uses a folder structure to hold different versions of the source code. The "single" format supports only one version of a file and identifies clones both within and across all files in a folder. This mode is best suited to the prepared dataset and the objectives of our study.

IClOnes was designed to work with Java, $\mathrm{C}++$, Ada, and Python, but its tokenbased matching mechanism ignores program structure, and allows it to be used with other languages. We were able to run it over JavaScript and PHP code snippets as well. An extension renaming is used to accomplish this. All .js and .php samples have been renamed to .java and .py, respectively. 


\subsubsection{Source Code Cleanup}

This step is optional, however it is advised by the ICLONES documentation to clean source code. The cleanup includes replacing tab characters with spaces, deleting invisible control characters, and assuring a new line at the end of each file. We used iClean to cleanup the source code. iClean is a Ruby script that cleans each file individually. This step only needs to be completed once, and we do not need to repeat it every time the parameter settings are changed.

\subsubsection{Running ICLONES}

ICLONES is written in Java and does not require any installation. We used a Linux system with a $2.2 \mathrm{GHz}$ Intel $\mathrm{CPU}$ and $32 \mathrm{~GB}$ RAM to run IClOnES. ICLONES, like other clone detector tools, has several parameters that must be adjusted. One of these parameters is language. We set this parameter to "java" if the input source codes were .java or .js, and "python" if they were .py or .php. The two most critical parameters that influence the results of ICLONES are minclone and minblock. One of the efforts in this study was determining the appropriate configuration for ICLONES to detect clones over our dataset.

Minclone impacts the minimum token length, which primarily reflects itself in the results by limiting the length of fragments found. A minclone value of 100, the default value of this parameter, would only accept a fragment length of 100 . The other key option, minblock, may have an impact on this length. The minimal length for near-miss tokens to be merged is specified by minblock. A minblock of 20 would continue to compare smaller tokens of length 20 and above even with a minclone of 100. This can alter the amount of clones detected by accepting fragments smaller than the minclone value, allowing for more clones to be detected than usual. Using a minblock of 0 prohibits tokens from merging and only allows exact matches. The 
minblock value has the greatest impact on iClone's runtime; the lower the minblock value, the longer the tool runs. We tested minblock in the range of $[0,5,10,15$, $20]$, and minclone in the range of $[5,10,15,20]$. The default values of the ICLONES parameters did not work well on our dataset because Stack Overflow code snippets are usually short. As a result, we must determine the optimal ICLONES configuration for our dataset.

When executing ICLONES, the outformat argument specifies the format in which ICLONES should return the results. Text, XML, and RCF are the three available formats. The RCF (Rich Clone Format) 95 is a proprietary clone detection result representation format with ability to be customised. RCF delivers the most detailed report on the detected clones compared to text and XML output types. ICLONES is able to find different clone fragments for a code pair based on the values set for minblock and minclone. Therefore, a code pair may contain several cloned fragments of various clone types. We saved all information about detected clone fragments over our dataset in a SQL table called IClonesResults. This table provides the following information: code pair ID, fragment ID, clone start and end line, clone start and end offset, file length, and length of clone. The detected clone types were used to answer the first research question and label our dataset.

\subsubsection{Evaluating ICLONES Results}

In order to evaluate how accurately ICLONES detects clones, we randomly selected 60 samples from code pairs with clones. For Java code pairs, we randomly selected five samples with Type I clones, five samples with Type II clones, and five samples with Type III clones. We repeated this selection process for other programming languages as well. Then, using the start and end lines of both Stack Overflow and GitHub code snippets, we extracted the cloned fragments and evaluated the correctness of the detected clone types. 

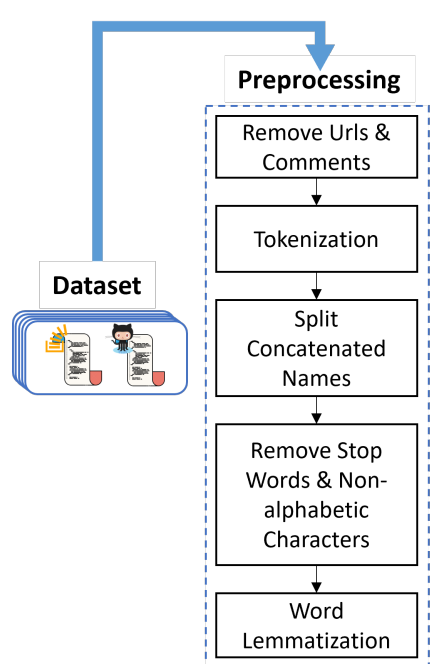

Lemmatization
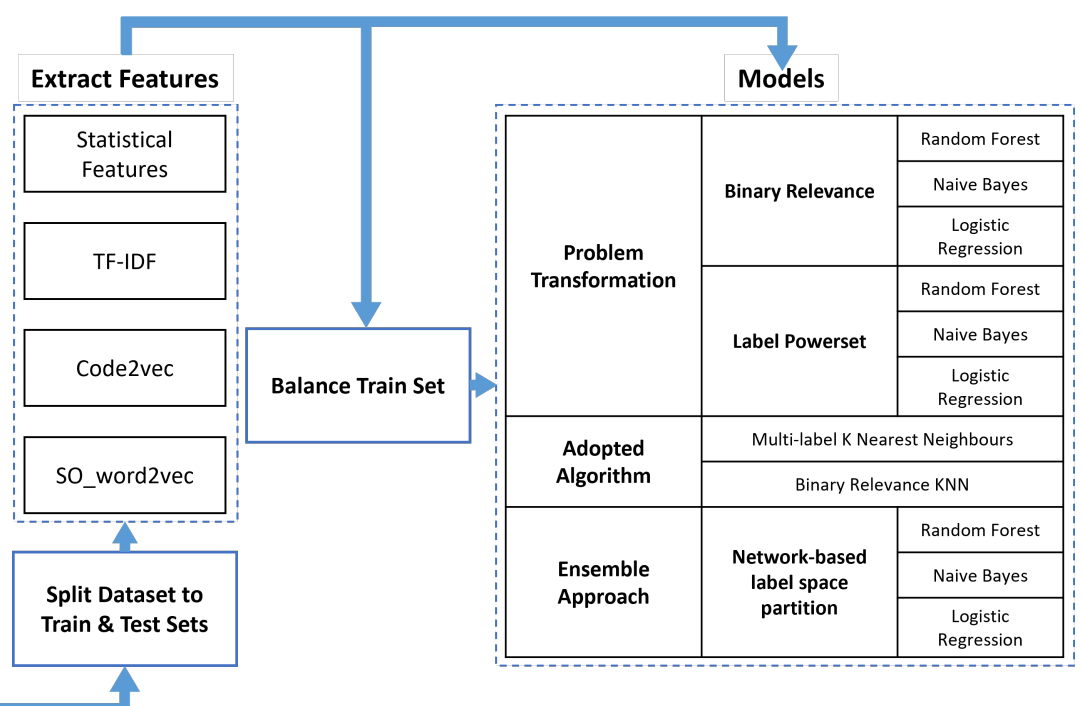

Figure 3.8: Overview of the process of predicting code clone types.

\subsection{Predicting Code Clone Types}

Figure 3.8 illustrates all steps of the process for predicting code clone types. The next sections present the details of these steps. We have 61,253 code pairs in four programming languages. The details of the dataset are discussed in Section 3.1. In order to learn a model that can predict clone types, we used the detected clone types (Section 3.2.3 as labels for the dataset. In addition, we considered our dataset as a corpus to extract four feature sets based on statistical features, TF-IDF, Code2Vec, and Word2Vec embedding models (refer to Section 3.3.4). One goal was to determine whether clone types are predictable. Another goal was to apply natural language processing techniques to extract features from the code. Once the features are extracted, we analyzed which feature sets have better predictive power.

\subsubsection{Preparing Dataset}

In order to prepare our dataset, we transformed Stack Overflow and GitHub code snippets in a Pandas dataframe which kept clone types between two code snippets if 


\begin{tabular}{|c|c|c|c|c|c|c|}
\hline Code Pair ID & Type 1 & Type 2 & Type 3 & No Clone & $\begin{array}{c}\text { SO Code } \\
\text { Snippet }\end{array}$ & $\begin{array}{c}\text { GH Code } \\
\text { Snippet }\end{array}$ \\
\hline
\end{tabular}

Figure 3.9: Dataset schema in Pandas.

any. Figure 3.9 shows the schema of this dataframe. "Code Pair ID" denotes a unique identifier that we assigned to each code pair in Section 3.1. "Type 1" is a binary variable with a "True" value, if any Type I code clone fragment appears in the code pair. Similarly, "Type 2" and "Type 3" indicate if any Type II and III cloned fragments exist. The "No Clone" variable is set to "True" if there is no cloned fragments between two code snippets. We considered these variables as target variables. In addition, we created a dictionary named "KeywordsDict" that includes all Java, JavaScript, PHP, and Python keywords. This dictionary is employed when pre-processing and extracting statistical features.

As shown in Figure 3.9 , each sample in our dataset may or may not contain clone fragments. If code fragments are present in a sample, they could be of Type I, II, III, or a combination of these. As samples can belong to more than one target variable, we deal with a multi-label classification problem.

\subsubsection{Data Cleaning And Pre-processing}

We intend to treat each code snippet as a natural language text, similar to the other NLP works; hence, we need to apply a pre-processing step. This procedure is carried out on all values of the $\mathrm{SO}$ code snippets and GH code snippets individually. A code snippet in our dataset contains 1 to $\mathrm{n}$ lines of codes which may contain comments, methods, and other code components. We eliminated comments from code snippets because they are not considered in the clone detection procedure, and none of the clone types rely on them. URLs do not carry useful information in our study as well. So, we removed them from the code snippets. Comments and URLs are removed with 
the help of re package in Python.

In source code, it is usual to name variables or procedures with concatenated words that indicate their function. The concatenated words are split down to make our code snippets more like a natural language text. For example, the method name "getResources" is separated into two words: "get" and "resources". At first, code snippets were tokenized by NLTK [96] and converted to lower case. After that, all non-keyword concatenated words were split by Wordninja [97. Wordninja uses a probabilistic model to split concatenated words via NLP, based on English Wikipedia ${ }^{1}$ unigram frequencies. All words with a length of less than two are ignored.

The next step has included removal of stop words and non-alphabetical words from the code snippets. Because stop words have no special meaning, we removed them using English stop words from the "nltk.corpus" package to save space and processing time. At the end of the pre-processing step, we use word lemmatization from NLTK package. The goal of the lemmatization step is to reduce the variation of words in our corpus. We found some empty Stack Overflow and GitHub code snippets after pre-processing. This was common with small code snippets that did not provide any valuable information. These pairs were not taken into consideration for the next steps. In addition, 'SO Code Snippet' and 'GH Code Snippet' columns were merged to a column named 'Content'. This column was used instead of the 'SO Code Snippet' and 'GH Code Snippet' columns in the following phases.

\subsubsection{Split Dataset}

Overfitting and underfitting are two common issues in machine learning. When a model performs exceptionally well on the data we used to train it, but fails to generalize effectively to new, unseen data points, this is known as overfitting. Underfitting, on the other hand, occurs when the model performs poorly even with the data that

\footnotetext{
${ }^{1}$ https://en.wikipedia.org/wiki/Wikipedia:About
} 
was used to train it. The most typical strategy for identifying these issues is to create separate data samples for training and testing the model. In this way, we can use the training set to train our model and then use the testing set to assess if the model can generalize well to unseen data. We divided our modelling dataset into two sets: training and testing, with $80 \%$ of samples are used for training and $20 \%$ for testing. In this case, we used the Scikit-learn library's train_test_split method.

In this study, apart from model performance, we must choose among various models and analyze which model performs better. Cross-validation is a re-sampling technique for evaluating machine learning models on a small set of data. The procedure has only one parameter, $\mathrm{k}$, which specifies the number of groups into which a given data sample should be divided. In this study, we used 5 -fold cross validation from Scikit-learn library in the training phase.

Prior to feature extraction, we partitioned our dataset into training and testing sets, because real-world data is represented by test data points. To avoid introducing future information to our model, we must run functions like fit() exclusively over the training instances in particular tasks like scaling or TF-IDF vectorizer.

\subsubsection{Feature Extraction}

After splitting the dataset, word vectorization methodologies were used to vectorize the 'Content' column on both the train and test datasets. Three separate feature sets are evaluated in this study: (i) statistical features that focused on the frequency of programming paradigms in code snippets, as well as two word vectorization techniques (ii) TF-IDF and (iii) word embedding. The aim of using these different feature sets is to compare their predictive ability in the clone type prediction problem.

When numerical input variables are scaled to a standard range, many machine learning algorithms perform better. Standardization is a technique for scaling numerical data prior to modeling. Each input variable is scaled independently by subtracting 
the mean and dividing by the standard deviation to shift the distribution, in order to have a standard deviation of one and a mean of zero. After extracting features, StandardScaler method from Scikit-learn library was applied on feature sets. We fitted it on the training dataset, and later transform test dataset with it.

Feature matrices are also compressed using the scipy.sparse package's lil_matrix method. It is a memory-efficient data structure for storing big matrices with a small number of nonzero entries.

\subsubsection{Statistical Features}

The features we retrieved from code snippets before applying pre-processing are referred to as statistical features in this study. They are extracted using software paradigms such as the amount of lines, keywords, and so on. These features are presented in Table 3.3, along with a description of each feature. Each feature is calculated separately for Stack Overflow and GitHub code snippets, then summed together. "Language" is a categorical feature with a value of [Java, JavaScript, PHP, Python]. We utilized the OneHotEncoder method from the Scikit-learn library to convert "Language" to a one-hot numeric array because categorical features were not supported in the balancing method we used in this study.

\subsubsection{TF-IDF}

TF-IDF is an abbreviation for Term Frequency Inverse Document Frequency. The TF-IDF is a statistical measure that assesses the relevance of a word to a document in a set of documents. This is accomplished by multiplying two metrics: (i) the number of times a word appears in a document and (ii) the word's inverse document frequency over a collection of documents.

Although TF-IDF has a variety of applications in information retrieval or keyword extraction, it has some limitations such as: (i) computing document similarity directly 


\begin{tabular}{lll}
\hline Name & Type & Description \\
\hline Language & Categorical & $\begin{array}{l}\text { Can be one of the Java, JavaScript, PHP, } \\
\text { or Python }\end{array}$ \\
\hline LineCNT & Integer & Number of lines \\
\hline CommentLineCNT & Integer & Number of comment lines \\
\hline KeywordCNT & Integer & $\begin{array}{l}\text { Total number of keywords in a code snip- } \\
\text { pet }\end{array}$ \\
\hline NumberCNT & Integer & The amount of numbers in a code snippet \\
\hline NameCNT & Integer & Total number of identifiers \\
\hline ConditionCNT & Integer & Number of if and if-else statements \\
\hline LoopCNT & Integer & Total number of for and while loops \\
\hline BracketCNT & Integer & Total number of brackets like "()[]\{\}" \\
\hline PunctCNT & Integer & Number of punctuation marks like ".;" \\
\hline OperatorCNT & Integer & Total number of operators like "+-*/" \\
\hline CompratorCNT & Integer & Total number of " $<>==<=>=<>!="$ \\
\hline
\end{tabular}

Table 3.3: Statistical features.

in the word-count space, which may be slow for large vocabularies, (ii) assuming that the number of words counted provides independent evidence of similarity, and (iii) not making use of word semantic similarity.

In this study to vectorize samples, we utilized the Scikit-learn library's TfidfVectorizer method. The following parameters of this method were validated and taken into consideration:

- min_df. When creating the vocabulary, it ignores terms with a document frequency that is lower than the given threshold. It accepts either float or integer values and has a default value of 1 . In this study, we set the threshold to 0.00009 .

- $\max$ features. This parameter's value can be an integer or 'None.' If 'None' 
is not specified, the word vectorizer creates a vocabulary that only takes into account the top max features that are sorted by term frequency across the corpus. In our study due to computational complexity, only the top 100 ranking features sorted by term frequency were considered.

- ngram_range. It determines the lower and upper bounds of the range of nvalues to be extracted for various n-grams. For instance, the n-gram range (1, 3) denotes unigram, bigram, and trigram. In this thesis, an n-gram range of $(1,3)$ is considered.

\subsubsection{Word Embeddings}

As the goal of this thesis is to improve predictive performance, another word vectorizer methodology, i.e., word embedding, was also applied. Word2Vec [98] is a popular technique for learning word embeddings. Word2Vec learns word associations from a vast corpus of text using neural network models. Once the corpus is trained, the Word2Vec model can find synonyms and even suggest extra words for a partial sentence. Word2Vec has an edge over TF-IDF in this regard. Furthermore, as the name implies, Word2Vec represents each unique word with a set of numbers known as vectors. One advantage of this paradigm is that a simple mathematical function like cosine similarity may be used to show the level of semantic similarity between the words represented by those vectors. Two pre-trained word embeddings from the software engineering domain were employed in this thesis to convert text to word vectors based on semantic similarity. We used Code2Vec [99] and Word2Vec [100 trained on the Stack Overflow data. These word embeddings are loaded into the model using Gensim 2, Gensim is an open source library used for natural language processing.

Code2Vec. It was developed by Alon et al. [99]. Code2Vec learned code embeddings, which are continuous vectors used to represent code snippets. The generated

\footnotetext{
${ }^{2}$ https://pypi.org/project/gensim
} 
code vectors can be fed into any machine learning pipeline that performs tasks like code retrieval, captioning, classification, and tagging, or utilized as a metric for comparing similarity between code snippets for ranking and clone detection. The authors used paths in the program's abstract syntax tree as their representation. They were able to capture regularities that reflect common code patterns by modelling a code snippet using its syntactic paths. They discovered that this format considerably reduces the learning burden while remaining scalable. Their pre-trained model mapped samples to a vector length of 128 .

Word2Vec. Efstathiou et al. [100] have applied Word2Vec to a corpus of 15GB of textual data drawn from Stack Overflow posts to train word embeddings targeted at the software engineering domain. They have called it SO-Word2Vec. They demonstrated how training on a domain-specific corpus allows their embeddings to capture the meaning of technical terms in a software engineering context that could not be captured when training on general-purpose text corpora. Their pre-trained model mapped samples to a vector length of 200 .

\subsubsection{Multi-label Classification Models}

After extracting features, we apply classification models on the multi-label dataset. There are three types of classification problems in machine learning:

1. Single label classification. in this case, we can have two (binary) or more (multiclass) labels, but each sample is assigned to one and only one label.

2. Multi-label classification. The goal is to learn from a collection of samples, each of which belongs to one or more classes based on a set of independent class labels.

3. Multi-output/task classification. This means that a single estimator is responsible for a number of joint classification tasks. This is a generalization of the 
multi-label and the multi-class classifications, where the set of classification problems is restricted to binary classification.

The second part of this thesis is focused on predicting the clone type of code pairs extracted from online platforms. Each code pair in our dataset could be made up of several cloned fragments with different clone types. One of the challenges in this study was selecting the most appropriate classification model. We had two choices:

1. Divide each code pair to its cloned fragments and consider our problem as a multi-class classification. Each fragment could assign to a label 'No Clone', 'Type 1', 'Type 2', or 'Type 3'. However, how to treat code pairs without clones becomes problematic in this scenario as we do not have samples representing no clone type.

2. Another scenario would be to consider each code pair as a sample. So, a sample in our dataset could belong to more than one label which are 'No Clone', or 'Type 1', 'Type 2', and 'Type 3'.

In this study, we considered the second option. Since using multi-label classification models to predict the labels of clones appears to be the closest to reality. A multi-label classification problem can be solved using one of the following three methods in the machine learning literature [101]:

1. Problem Transformation Method. In this method, we try to transform our multi-label problem into single-label problems. This method can be carried out in two different ways as:

(a) Binary relevance. In this approach each label is treated independently and the model is simplified to n classification problems. the main drawback for this technique is ignoring the correlation between labels by treating each 
label independently. As in our study there is no correlation among labels, it had no effect.

(b) Label Powerset. In this approach the problem is transformed into a multi-class problem with a single multi-class classifier trained on all unique label combinations identified in the training data. Despite the fact that this method takes label correlation into account, the result may be an unbalanced dataset with many classes. In other words, the principal disadvantage of this approach is that as the number of labels grows, the number of classes or subsets grows exponentially. This would result in a dataset that is sparse and unbalanced [102].

2. Algorithm Adaption. Adapted algorithm, as the name implies, adapts the algorithm to conduct multi-label classification directly rather than transforming the problem into distinct subsets. Multi-label K Nearest Neighbours (MLKNN) 103 and Binary Relevance K Nearest Neighbours (BRKNN) 104 are two algorithms in this regard. MLKNN uses k-Nearest Neighbors to find nearest examples to a test class and uses Bayesian inference to predict labels. This is a distance-based method and works well when there is a relationship between distance and labels. BRkNN is a multilabel classification adaptation of the $\mathrm{kNN}$ algorithm that is conceptually equivalent to employing the popular Binary Relevance problem transformation approach with the kNN algorithm, but significantly faster.

3. Ensemble Method. Partition label space and classify each subspace separately is an ensemble method 105. This classifier performs classification by partitioning the label space into separate, smaller multi-label sub-problems, using the supplied label space cluster. Then, it trains an instance of the supplied base multi-label classifier for each label space subset in the partition. And at 
the end, it predicts the result with each of sub-classifiers and returning the sum of their results.

After that, for each of the methods excluding algorithm adoption, the thesis employed one of the three base algorithms [106]:

1. Gaussian Naive Bayes. This is a probabilistic machine learning model that is used for classification task based on the Bayes theorem. The classifier's features/predictors indicate the frequency of the words in the document. Furthermore, it assumes when the predictors take on a continuous value (rather than discrete values), the values are sampled from a Gaussian distribution. Because our dataset only contains numeric and continuous features, we picked Gaussian Naive Bayes as our model.

2. Logistic Regression. It is an approach for predicting the probability of a categorical dependent variable using machine learning. Logistic regression can be binomial or multinomial. Binomial logistic regression is used in our scenario. The dependent variable in logistic regression is a binary variable that comprises data coded as 1 or 0 . It means, as a function of $\mathrm{X}$, the logistic regression model predicts $\mathrm{P}(\mathrm{Y}=1)$. However, some assumptions should be taken into account before using logistic regression such as no missing values in the dataset, binary labels, and no correlation among data variables.

3. Random Forest. Random forest is an ensemble machine learning algorithm that performs well or very well in a variety of classification and regression predictive modelling problems. It is made up of a number of independent decision trees that work together as a unit. Each tree in the random forest produces a class prediction, and the class with the most votes becomes the prediction of the model. It uses bagging and feature randomness when building each individual 
tree to try to create an uncorrelated forest of trees. Because a huge number of highly uncorrelated trees acting as a committee, the random forest model works so effectively. However, in order for random forest to be able to make accurate class predictions, it needs features that have at least some predictive power, and the trees of the forest be uncorrelated or at least have low correlations with each other.

\subsubsection{Balancing Multi-label Classification}

The number of samples associated with classes in a classification problem is not equal and in the real-world, the number of samples in a class may significantly be lower than that of the other and resulting in a data imbalance problem. In most problems, unbalancing has a significant impact on the performance of machine learning algorithms. Our dataset is also imbalanced, with labels spread unevenly. In this study, we use MLSMOTE [107, a multi-label balancing technique, to investigate the effects of balancing in our problem.

MLSMOTE or Multilabel Synthetic Minority Over-sampling Technique is used to handle data imbalance in multi-label classification. It is one of the most common and effective data augmentation approaches. MLSMOTE is a variation or extension of the basic SMOTE [108]. SMOTE augments the majority class labels alongside the minority class labels. As in a multi-label problem each instance of the data is associated with numerous labels, applying SMOTE to a multi-label classification model is a challenge. MLSMOTE tries to solve this problem by picking the right data points, particularly those with minority labels. The feature vectors corresponding to the minority label data are then generated with new additional data. Labels in the majority are referred to as head labels, while labels in the minority are referred to as tail labels in multi-label contexts. The MLSMOTE steps are briefly outlined as follows: 
1. Select data with minority class labels to over-sample. Because it is more likely that more than one label in multi-label data will be a tail label, proper criteria for picking minority labels should be established.

2. Select a data instance that requires augmentation.

3. Determine the data point's nearest $\mathrm{k}$ neighbours.

4. Make a synthetic data point anywhere on the line connecting the two points using a random data point that is in $\mathrm{k}$ nearest neighbours of the selected data point.

5. Repeat the procedure until the data is evenly distributed.

\subsubsection{Evaluation Metrics}

In the field of machine learning, evaluation metrics are extremely important. For single label classification problems, there are standard evaluation metrics such as precision, recall, F1-Score, and ROC. However, single label evaluation measures are usually different from multi-label evaluation methods. In multi-label classification, a misclassification is no longer a hard wrong or right. A prediction that contains a subset of the actual classes is preferable to one that has none of them; for example, correctly predicting two of the three labels is preferable to correctly predicting none of the labels at all. This makes multi-label classifier evaluation more difficult than single-label classifier evaluation, so single-label metrics must be correctly updated to perform model evaluation [109]. Regardless, F1-score and Hamming Loss are the most essential evaluation metrics for multi-label models.

We must average out the classes in order to measure a multi-class classifier. Microaveraging and macro-averaging are two possible methods for doing this. In microaveraging all true positives, true negatives, false positives and false negatives for 
each class are summed up and then the average is taken. While in macro-averaging method, the individual true positives, false positives, and false negatives of the system are summed up for different sets and then apply them. When we want to know how the system performs overall across sets of data, we can utilize the macro-averaging method. On the other hand, micro-averaging can be a useful measure when dataset varies in size. In this study, we used micro-averaging method. Like single label classification, in multi-label classification, higher value of precision, recall, and F1score show better performance of a learning algorithm.

Precision (Prc): is the proportion of predicted correct labels to the total number of actual labels, averaged over all instances.

$$
\operatorname{Prc}^{\text {micro }}=\frac{1}{n} \sum_{i=1}^{n} \frac{\left|y_{i} \cap \widehat{y_{i}}\right|}{\left|y_{i}\right|}
$$

Recall (Rcl): is the proportion of predicted correct labels to the total number of predicted labels, averaged over all instances.

$$
\operatorname{Prc}^{\text {micro }}=\frac{1}{n} \sum_{i=1}^{n} \frac{\left|y_{i} \cap \widehat{y}_{i}\right|}{\left|\widehat{y}_{i}\right|}
$$

F1-score (F1): F1-measure is the harmonic mean of precision and recall.

$$
F 1^{\text {micro }}=\frac{1}{n} \sum_{i=1}^{n} \frac{2 \times\left|y_{i} \cap \widehat{y_{i}}\right|}{\left|y_{i}\right|+\left|\widehat{y}_{i}\right|}
$$

Hammin Loss (HL): Hamming Loss is the fraction of incorrectly predicted class labels to the total number of actual labels. In case of all the correctly classified tags, Hamming Loss will be 0 .

$$
H L=\frac{1}{k n} \sum_{i=1}^{n} \sum_{j=1}^{k}\left[I\left(l \in \widehat{Y}_{i} \wedge l \notin Y_{i}\right)+I\left(l \notin \widehat{Y_{i}} \wedge l \in Y_{i}\right)\right]
$$

Where, $I$ is the indicator function and $T$ is a multi-label dataset consisting $n$ 
multi-label examples $\left(x_{i} ; Y_{i}\right), 1 \leq i \leq n, x_{i} \in x, y_{i} \in y, y=0,1^{k}$; with a label-set $l ;|l|=k$. And, $h$ is a multi-label classifier and $z_{i}=h\left(x_{i}\right)=0,1^{k}$ is the set of label memberships predicted by $\mathrm{h}$ for the sample $x_{i}$ [109]. 


\section{Chapter 4}

\section{Results}

The findings of experiments are reported in this chapter with the goal of answering all of the research questions from Section 1.3 . This chapter is organised as follows. The results for research questions one and two are reported in Section 4.1. Section 4.2 presents the results for answering research question three and determining whether or not code clone type is predictable.

\subsection{Code Clone Detection}

In this section, we discuss the results obtained for detecting clones to answer the first and second research questions. Section 4.1.1 discusses our efforts to obtain more accurate results from ICLONES. We answer the first research question related to how accurately clone detection tools can identify code clones between Stack Overflow and GitHub in this section. Also, we present the results of manual code clone detection assessment in this section. The manual inspection results establish a baseline for evaluating the selected tool, ICLONES. In addition, the answer to the second research question that investigates what types of clones are present in the GitHub code snippets that are reused from Stack Overflow, and the ICLONES results are presented in Section 4.1.2. 


\begin{tabular}{lllllc}
\hline Clone Type & \multicolumn{1}{c}{ Java } & JavaScript & \multicolumn{1}{c}{ PHP } & \multicolumn{1}{c}{ Python } & Total \\
\hline Type I & $7(14.58 \%)$ & $10(20.83 \%)$ & $9(18.75 \%)$ & $9(18.75 \%)$ & $\mathbf{3 5}(\mathbf{1 8 . 2 2 \%})$ \\
\hline Type II & $6(12.5 \%)$ & $7(14.58 \%)$ & $3(6.25 \%)$ & $6(12.5 \%)$ & $\mathbf{2 2}(\mathbf{1 1 . 4 6 \% )}$ \\
\hline Type III & $20(41.67 \%)$ & $14(29.17 \%)$ & $17(35.42 \%)$ & $17(35.42 \%)$ & $\mathbf{6 8}(\mathbf{3 5 . 4 2 \%})$ \\
\hline N/A & $15(31.25 \%)$ & $17(35.42 \%)$ & $19(39.58 \%)$ & $16(33.33 \%)$ & $\mathbf{6 7}(\mathbf{3 4 . 9 \% )}$ \\
\hline
\end{tabular}

Table 4.1: Summary of the manual clone detection process.

\subsubsection{RQ1: How Accurately Can Clone Detection Tools Iden- tify Code Clones Between Stack Overflow and GitHub?}

Manual Assessment of Code Clone Detection. IClOnes requires two critical parameters to be adjusted depending on the dataset. To determine a baseline for the most optimal ICLONES' configuration, we randomly selected 192 samples and distributed them among 26 participants (i.e., volunteer students) in order to manually detect clone types. The results of the manual clone detection process are presented in Table 4.1. If we exclude samples without clones, which account for $35.9 \%$ of all samples, Type III is the most common clone type, accounting for $35.42 \%$ of all samples, and Type I is the second most common, representing $18.22 \%$ of all samples. Type II has the fewest samples of any programming language, accounting for $11.46 \%$ of all samples.

Furthermore, we calculated Cohen's Kappa coefficient as a measure of agreement between two coders. The Cohen's Kappa coefficient is +0.76 indicating a substantial agreement between coders. The participants detected similar clones in 156 code pairs out of 192. We (the author of this thesis) resolved the conflicts by analyzing participants' rationale, manually verifying their labels and making the final decision on the clone type(s) for the remaining 36 code pairs with no agreement.

IClones' Configuration. Minblock and minclone are the two most important ICLONES's parameters that influence the results. We tested minblock in the $[0,5,10$, 
$15,20]$ range and minclone in the $[5,10,15,20]$ range; in total we ran 13 different configurations on selected samples to determine the most optimal configuration of the tool for our dataset. Minclone defines the minimal length required for a fragment to be accepted. And, the minimal length for near-miss tokens to be merged is specified by minblock. A minblock of 0 prohibits tokens from merging and only allows exact matches. The overall percentage of detected clones for the selected samples is shown in Table A.1, while Figure 4.1 depicts the percentage trend of clone detection for each programming language separately. In addition, Figures 4.2, 4.3, 4.4, and 4.5 display the detected clone types for Java, JavaScript, PHP, and Python, respectively.

As Figure 4.1 shows, the percentage of detected clones is decreasing when we increase the minclone and minblock values. The average of the detected clones has decreased from $77.08 \%$ to $27.08 \%$. This tendency demonstrates that higher values do not detect clones, and the default values of these parameters, i.e., 100 and 20 for minclone and minblock, do not detect clones over the selected samples. However, according to Figures 4.2 to 4.5 , with a small value of minclone and minblock ICLONES tends to detect Type I clones. We compared these results to those detected by human subjects to gain a better understanding of the accuracy of configurations.

Table 4.2 shows the ratio of similarity between different ICLONES configurations and detected clones by human subjects. The most similar result was achieved by the minblock $=5$ and minclone $=10$ that is $28.65 \%$. Furthermore, as shown in Figures 4.2 to 4.5 , the ratio of detected clone types is more similar to the ratio of detected clone types by human subjects, since with other configurations some types are disregarded or detected far lower than reality. Based on these results, we have determined minblock $=5$ and minclone $=10$ as our optimal configuration and run ICLONES with these parameters across all samples in our dataset.

We chose 15 samples at random from the detected clones in each programming language to manually evaluate how well ICLONES detects clones. We selected five 


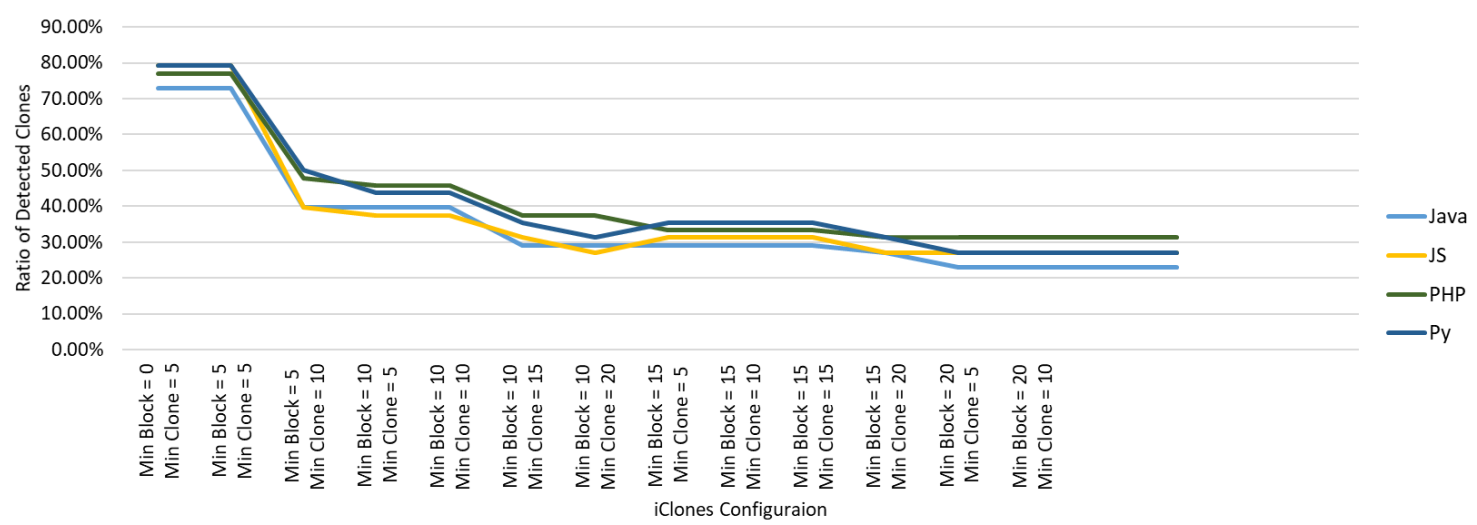

Figure 4.1: Ratio of detected clones for different configurations of ICLONES.

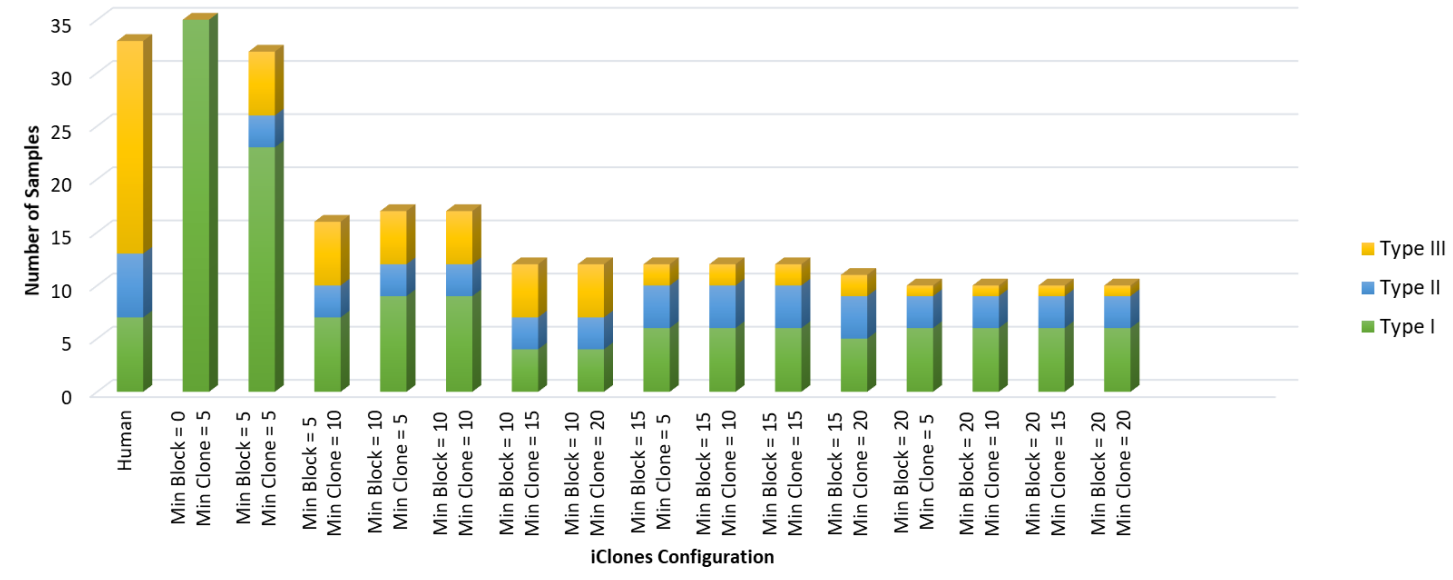

Figure 4.2: Number of detected clones in Java samples.

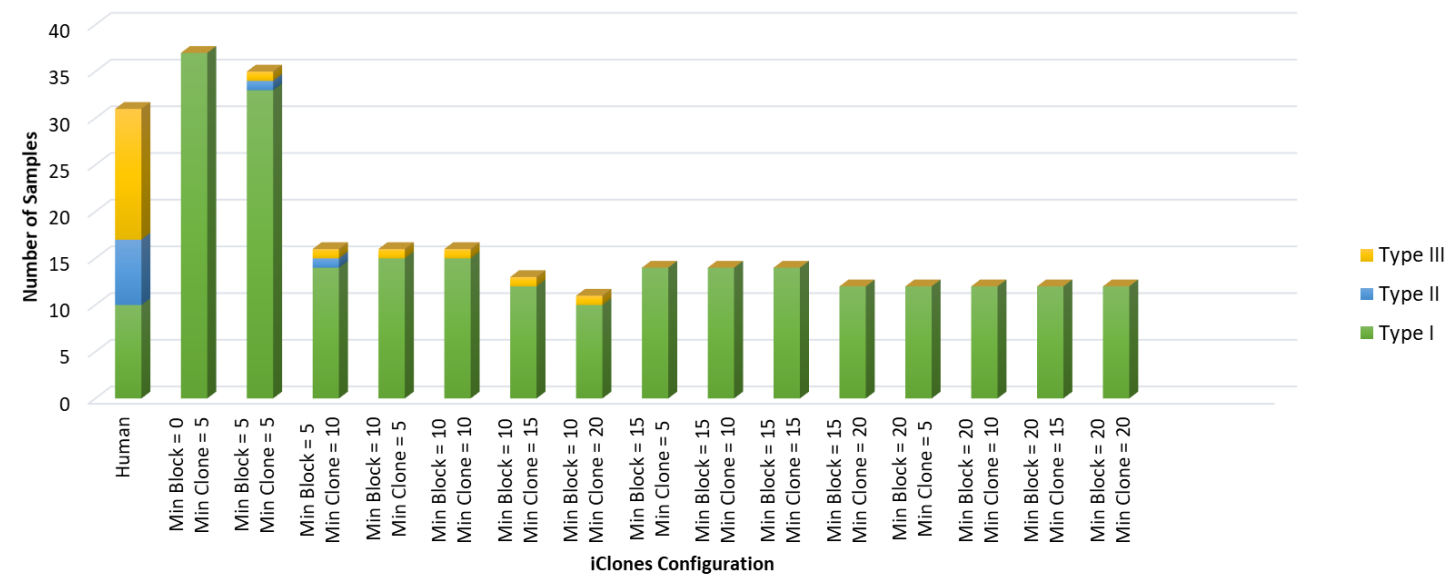

Figure 4.3: Number of detected clones in JavaScript samples. 


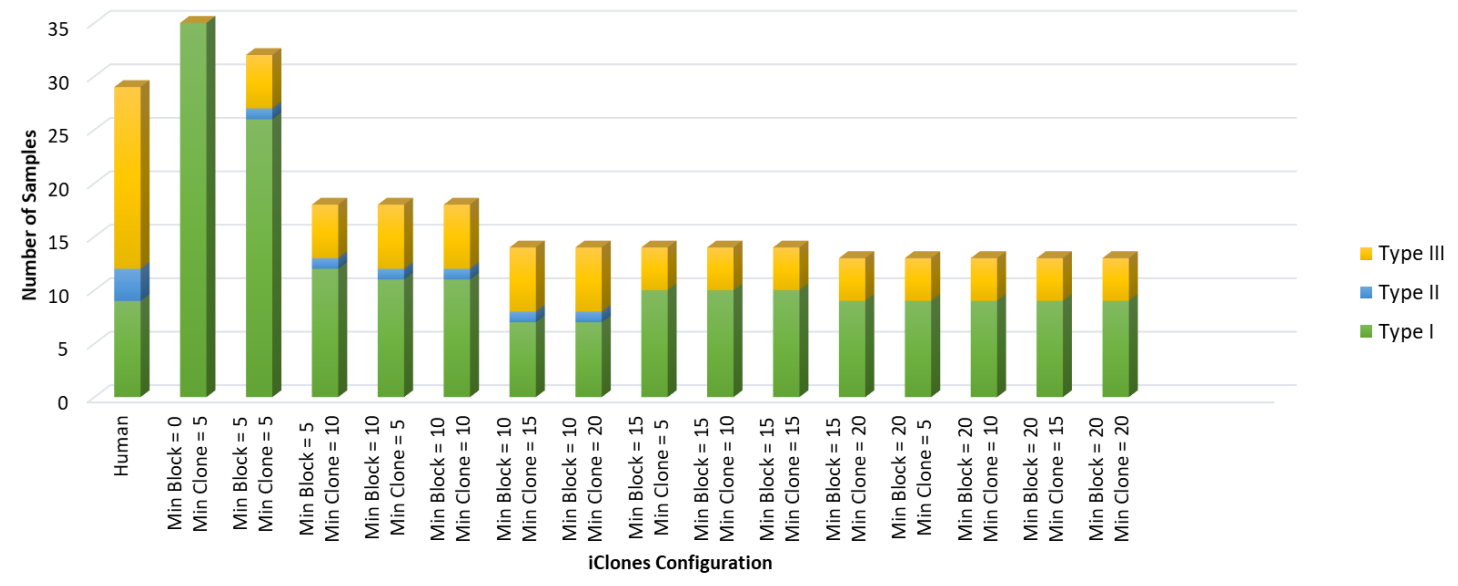

Figure 4.4: Number of detected clones in PHP samples.

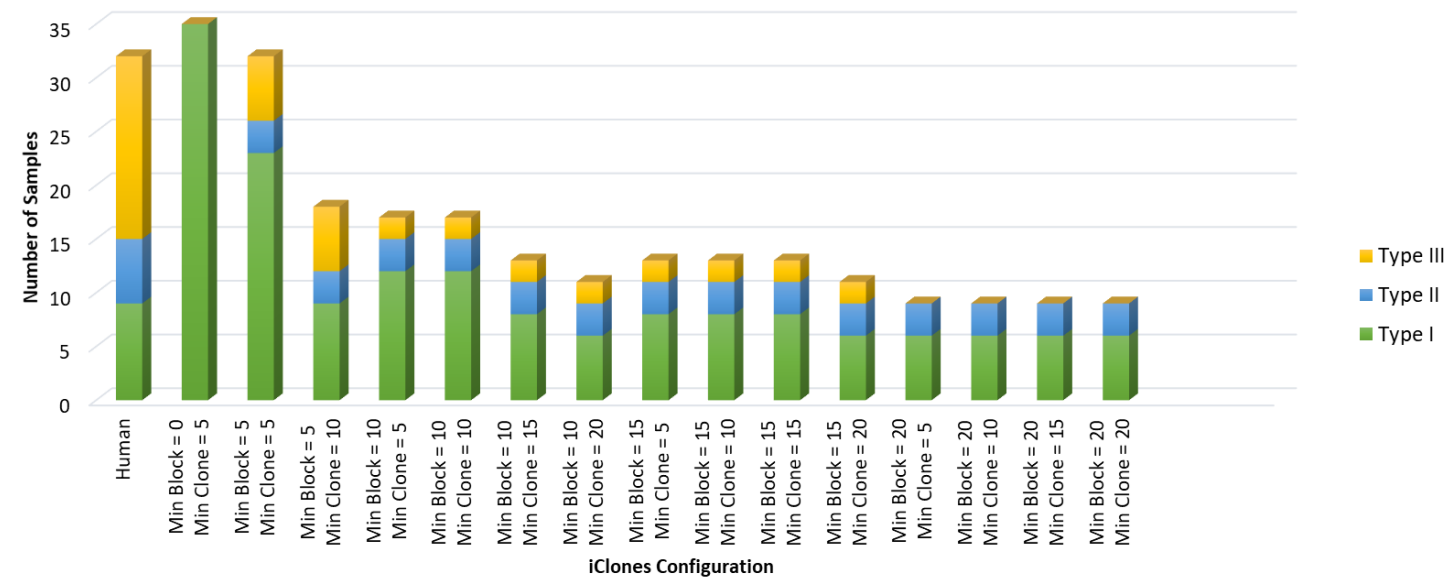

Figure 4.5: Number of detected clones in Python samples. 


\begin{tabular}{|c|c|c|c|c|c|}
\hline Configuration & Java & JS & PHP & Py & Avg \\
\hline $\begin{array}{l}\text { Min Block }=0 \\
\text { Min Clone }=5\end{array}$ & $20.83 \%$ & $20.83 \%$ & $22.92 \%$ & $18.75 \%$ & $20.83 \%$ \\
\hline $\begin{array}{l}\text { Min Block }=5 \\
\text { Min Clone }=5\end{array}$ & $29.17 \%$ & $22.92 \%$ & $35.42 \%$ & $22.92 \%$ & $27.60 \%$ \\
\hline $\begin{aligned} \text { Min Block } & =5 \\
\text { Min Clone } & =10\end{aligned}$ & $29.17 \%$ & $22.92 \%$ & $37.50 \%$ & $25.00 \%$ & $28.65 \%$ \\
\hline $\begin{aligned} \text { Min Block } & =10 \\
\text { Min Clone } & =5\end{aligned}$ & $27.08 \%$ & $20.83 \%$ & $39.58 \%$ & $22.92 \%$ & $27.60 \%$ \\
\hline $\begin{array}{l}\text { Min Block }=10 \\
\text { Min Clone }=10\end{array}$ & $27.08 \%$ & $20.83 \%$ & $39.58 \%$ & $22.92 \%$ & $27.60 \%$ \\
\hline $\begin{array}{l}\text { Min Block }=10 \\
\text { Min Clone }=15\end{array}$ & $25.00 \%$ & $22.92 \%$ & $39.58 \%$ & $20.83 \%$ & $27.08 \%$ \\
\hline $\begin{array}{l}\text { Min Block }=10 \\
\text { Min Clone }=20\end{array}$ & $25.00 \%$ & $22.92 \%$ & $39.58 \%$ & $18.75 \%$ & $26.56 \%$ \\
\hline $\begin{array}{c}\text { Min Block }=15 \\
\text { Min Clone }=5\end{array}$ & $22.92 \%$ & $22.92 \%$ & $31.25 \%$ & $20.83 \%$ & $24.48 \%$ \\
\hline $\begin{array}{l}\text { Min Block }=15 \\
\text { Min Clone }=10\end{array}$ & $22.92 \%$ & $22.92 \%$ & $31.25 \%$ & $20.83 \%$ & $24.48 \%$ \\
\hline $\begin{array}{l}\text { Min Block }=15 \\
\text { Min Clone }=15\end{array}$ & $22.92 \%$ & $22.92 \%$ & $31.25 \%$ & $20.83 \%$ & $24.48 \%$ \\
\hline $\begin{array}{l}\text { Min Block }=15 \\
\text { Min Clone }=20\end{array}$ & $22.92 \%$ & $22.92 \%$ & $31.25 \%$ & $18.75 \%$ & $23.96 \%$ \\
\hline $\begin{aligned} \text { Min Block } & =20 \\
\text { Min Clone } & =5\end{aligned}$ & $22.92 \%$ & $22.92 \%$ & $31.25 \%$ & $18.75 \%$ & $23.96 \%$ \\
\hline $\begin{array}{l}\text { Min Block }=20 \\
\text { Min Clone }=10\end{array}$ & $22.92 \%$ & $22.92 \%$ & $31.25 \%$ & $18.75 \%$ & $23.96 \%$ \\
\hline $\begin{array}{l}\text { Min Block }=20 \\
\text { Min Clone }=15\end{array}$ & $22.92 \%$ & $22.92 \%$ & $31.25 \%$ & $18.75 \%$ & $23.96 \%$ \\
\hline $\begin{array}{l}\text { Min Block }=20 \\
\text { Min Clone }=20\end{array}$ & $22.92 \%$ & $22.92 \%$ & $31.25 \%$ & $18.75 \%$ & $23.96 \%$ \\
\hline
\end{tabular}

Table 4.2: Similarity ratio of ICLONES with manually detected clones by human subjects. 


\begin{tabular}{ccccc}
\hline & Type I & Type II & Type III & Total \\
\hline Java & $5(100 \%)$ & $4(80 \%)$ & $5(100 \%)$ & $\mathbf{1 4}(\mathbf{9 3 . 3 \% )}$ \\
\hline JS & $4(80 \%)$ & $5(100 \%)$ & $5(100 \%)$ & $\mathbf{1 4 ( 9 3 . 3 \% )}$ \\
\hline PHP & $4(80 \%)$ & $4(80 \%)$ & $5(100 \%)$ & $\mathbf{1 3 ( 8 6 . 7 )}$ \\
\hline Py & $5(100 \%)$ & $5(100 \%)$ & $4(80 \%)$ & $\mathbf{1 4 ( 9 3 . 3 \% )}$ \\
\hline Total & $\mathbf{1 8 ( 9 0 \% )}$ & $\mathbf{1 8 ( 9 0 \% )}$ & $\mathbf{1 9 ( 9 5 \% )}$ & $\mathbf{5 5 ( 9 1 . 7 \% )}$ \\
\hline
\end{tabular}

Table 4.3: Evaluation of the clone detection results on 60 randomly selected samples.

samples for each clone type and programming language. For example, we chose five samples at random from the detected clones that have Java as programming language and Type I code clones. As shown in Table 4.3 , the accuracy of clone detection was greater than $80 \%$ and, overall, it was around $91.7 \%$. The detection of Type III clones had an accuracy around 95\%, while for two other clone types it was $90 \%$.

Answer to RQ1: Many existing clone detection tools are unsuitable for Stack Overflow code snippets since these snippets are short and, in most cases, incomplete code blocks. However, IClONES, a token-based clone detector, is capable of parsing such code snippets. Our evaluation results reveal that IClONES can detect Type I, Type II and Type III code clones with the overall accuracy of $91 \%$ across four programming languages.

\subsubsection{RQ2: What Types of Clones Are Present in the Code Snippets Reused From Stack Overflow in the GitHub Projects?}

Figure 4.6 demonstrates the ratio of detected clones and code pairs without clones obtained after running ICLONES across our dataset. A considerable proportion of the samples, approximately $78 \%$, contain no clones. But, around $15.85 \%, 5.89 \%$, and 11.71\% samples contain Type I, Type II, or Type III clones, respectively. Table 4.4 


\begin{tabular}{|c|c|c|c|c|c|}
\hline & Java & JavaScript & PHP & Python & Total \\
\hline Type I & $723(16.95 \%)$ & 7,229 (16.64\%) & $522(19.16 \%)$ & $1,232(11.38 \%)$ & $9,706(15.85 \%)$ \\
\hline Type II & $331(7.76 \%)$ & $2,623(6.04 \%)$ & $184(6.75 \%)$ & $468(4.32 \%)$ & $3,606(5.89 \%)$ \\
\hline Type III & $472(11.06 \%)$ & $5,137(11.83 \%)$ & $427(15.68 \%)$ & $1,134(10.47 \%)$ & $7,170(11.71 \%)$ \\
\hline No Clone & $3,282(76.93 \%)$ & $32,864(75.66 \%)$ & $2,020(74.16 \%)$ & $8,945(82.61 \%)$ & $47,111(76.91 \%)$ \\
\hline Total & $4,266(100 \%)$ & $43,435(100 \%)$ & $2,724(100 \%)$ & $10,828(100 \%)$ & $61,253(100 \%)$ \\
\hline
\end{tabular}

Table 4.4: Summary of IClONES' clone detection results.

reports the clone detection results of ICLONES for each programming language in our dataset, while Figure 4.7 illustrates these results.

Among the programming language, PHP has the most Type I clones which is around $19.16 \%$ of its samples. While Type I clones amount to $16.5 \%$ for both Java and JavaScript and about $11 \%$ for Python. Java has the highest percentage of samples (7.76\%) for Type II clones, whereas Python has the lowest (4.32\%). The results for Type III are similar to Type I. PHP has the most samples for Type III clones, with $15.68 \%$, while JavaScript, Java, and Python follow it with $11.83 \%, 11.06 \%$, and $10.47 \%$, respectively.

Python has the highest percentage of samples without clones, at $82.61 \%$. This amount is similar for other programming languages which is $76.93 \%, 75.66 \%$, and $74.16 \%$ for Java, JavaScript, and PHP, respectively.

Since IClONES can detect multiple clone types for a code pair, we noticed that some samples in our dataset had more than one label (i.e., presence of several clone types in a pair). According to Table 4.5, 44.5\% of the 19,771 code pairs with clones had one clone type, $36.56 \%$ contained two different clone types, and $18.74 \%$ had all three clone types. This is further illustrated in Figure 4.8. Type I is the dominant type with 4,884 (24.7\%) samples, followed by 4,752 (24.04\%) samples with both "Type I and Type III". 3,705 (18.74\%) samples have all three clone types, while 2,805 (14.19\%) 


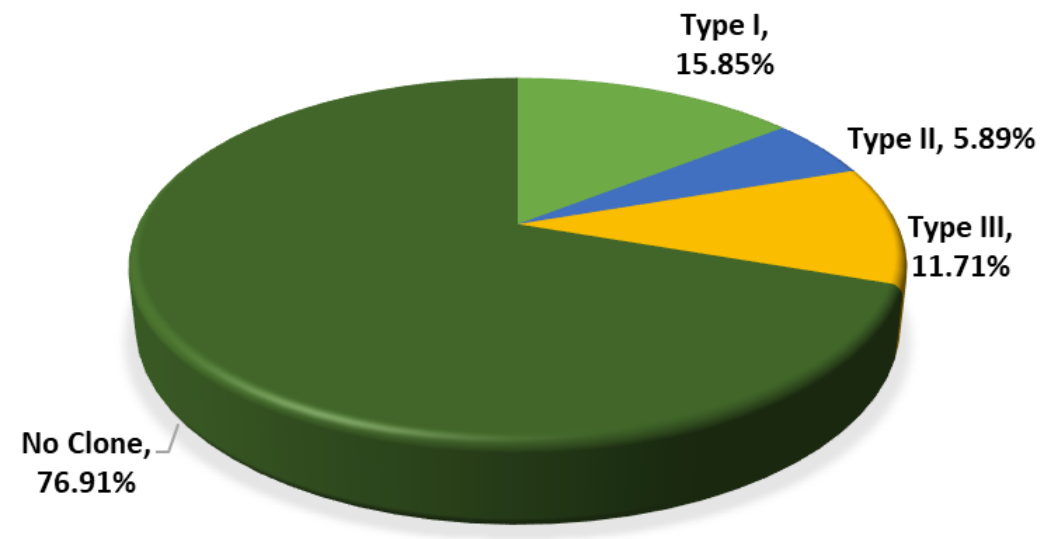

Figure 4.6: Ratio of detected clones by ICLONES.
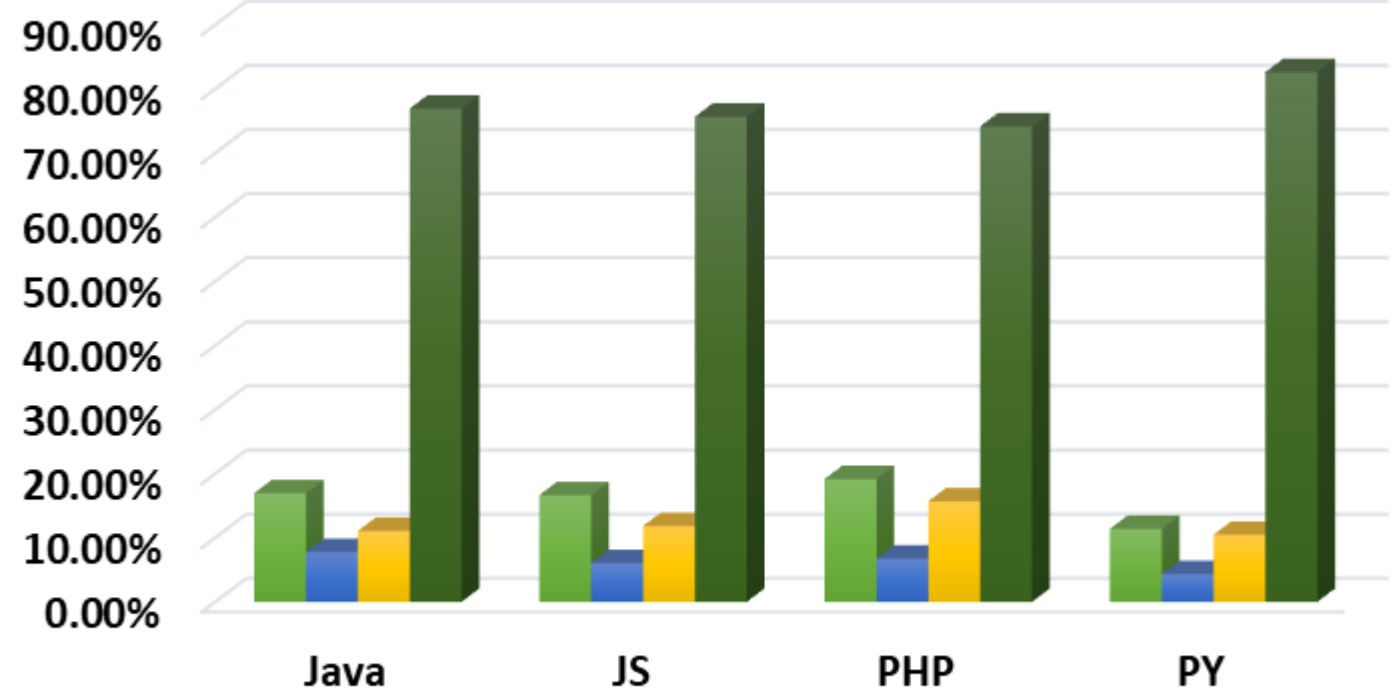

Type I Type II Type III No Clone

Figure 4.7: Ratio of detected clones for each programming language. 


\begin{tabular}{|c|c|c|c|c|c|}
\hline & Java & JavaScript & PHP & Python & Total \\
\hline One Clone Type & $547(35.85 \%)$ & $6,788(47.50 \%)$ & $338(29.38 \%)$ & $1,125(39.89 \%)$ & $8,798(44.50 \%)$ \\
\hline Two Clone Types & $664(43.51 \%)$ & $4,894(34.24 \%)$ & $606(53.49 \%)$ & $1,104(39.15 \%)$ & $7,268(36.56 \%)$ \\
\hline Three Clone Types & $315(20.64 \%)$ & $2,610(18.26 \%)$ & $189(16.68 \%)$ & $591(20.96 \%)$ & $3,705(18.74 \%)$ \\
\hline Total & $1,526(100 \%)$ & $14,292(100 \%)$ & $1,133(100 \%)$ & $2,820(100 \%)$ & $19,771(100 \%)$ \\
\hline
\end{tabular}

Table 4.5: Distribution of multi-clone types in our sample.

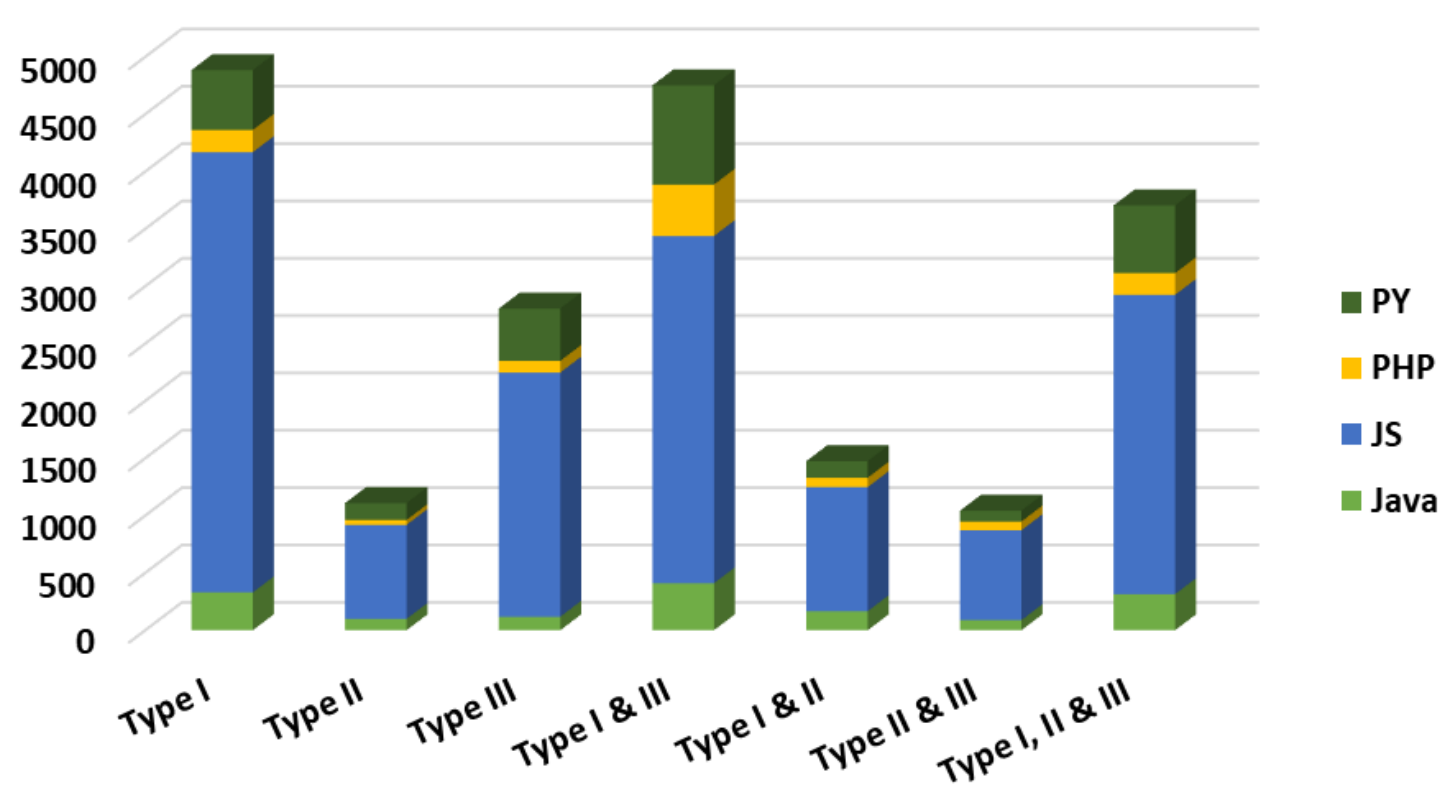

Figure 4.8: Distribution of labels.

samples have just Type III clones. In comparison with other clones, Type II clones and their combination with other clone types have the fewest samples. "Type I and Type II", "Type II", and "Type II and Type III" clones are found in 1,474 (7.46\%), $1,109(5.61 \%)$, and 1,042 (5.27\%) samples, respectively. 
Answer to RQ2: Our findings show that around $76.9 \%$ of the code pairs in our dataset had no clones detected. This suggests that developers do not directly copy code snippets, but rather leverage the shared help/knowledge from Stack Overflow in their own projects. On the other hand, among the code pairs with clones, Type I (15.85\%) and Type III (11.71\%) are the most frequently detected clones between Stack Overflow and GitHub code snippets. While, Type II (5.89\%) is a less common type of clones. This can imply that when developers do reuse code from Stack Overflow, they copy-paste snippets more often than they adapt them to their GitHub projects. Moreover, we found that $55.5 \%$ of code pairs contain more than one clone type. According to the findings, $44.5 \%$ of code pairs had one clone type, while $36.56 \%$ and $18.74 \%$, respectively, had two and three different clone types. This shows that developers adopt various strategies of code reuse.

\subsection{Code Clone Prediction}

This section provides the results of multi-label classification prediction, or in other words, it attempts to predict code clone types. The overall goal of this research question is to determine which feature set and which classifier are more powerful in detecting clone types. We went through various steps to answer the third research question. In Section 4.2.1, we describe the dataset once we pre-processed and split it to train and test sets. Feature sets are extracted after the dataset has been split. In Section 4.2.2, we apply two word vectorization approaches, extract TF-IDF and word embedding features in addition to statistical ones. Section 4.2.3 and Section 4.2.4 then discuss the performance of various classifiers across all feature sets. This is done before and after the dataset balancing. To balance the dataset, we synthetically augmented it by applying the MLSMOTE approach and under-sampling technique. 


\begin{tabular}{|c|c|c|c|c|c|}
\hline & Java & JS & PHP & PY & Total \\
\hline Type I & $723(17.54 \%)$ & $6,764(16.35 \%)$ & $522(19.83 \%)$ & $1,223(11.45 \%)$ & $9,232(15.70 \%)$ \\
\hline Type II & $331(8.03 \%)$ & $2,623(6.34 \%)$ & $184(6.99 \%)$ & $464(4.34 \%)$ & $3,602(6.13 \%)$ \\
\hline Туре III & $472(11.45 \%)$ & $4,905(11.86 \%)$ & $427(16.22 \%)$ & $1,133(10.61 \%)$ & $6,937(11.80 \%)$ \\
\hline No Clone & $3,137(76.12 \%)$ & $31,253(75.57 \%)$ & $1,929(73.26 \%)$ & $8,805(82.45 \%)$ & $45,124(76.75 \%)$ \\
\hline Total & $4,121(100 \%)$ & $41,358(100 \%)$ & $2,633(100 \%)$ & $10,679(100 \%)$ & $58,791(100 \%)$ \\
\hline
\end{tabular}

Table 4.6: Distribution of clone types for each programming language after preprocessing.

\subsubsection{Dataset Overview After Pre-processing and Splitting}

Some Stack Overflow or GitHub code snippets received a "Null" value after preprocessing; here we refer them as Null values. They were especially apparent in the short code snippets that lost all content after deleting stop-words and non-alpha words. In the feature extraction step, we ignored these samples. Table 4.6 displays the number of code pairs after deleting Null values from our dataset. As can be seen, the total number of samples dropped from 61,253 to 58,791 . When comparing Table 4.4 and Table 4.6, we discovered that code pairs with no clones contained more Null values than other cases. There was no Null value in Java and PHP code pairs that contained clones. But, this amount was 697 for JavaScript and 14 for Python.

Splitting the dataset into train and test sets is another step before extracting features. We considered $80 \%$ of the samples for train and $20 \%$ for test set. The amount of samples in the train and test sets for each programming language and the label distribution in them are shown in Table 4.7. Furthermore, the results in Table 4.8 ensure that the data is divided in the two sets with the same distribution, and one type does not dominate the others. 


\begin{tabular}{|c|c|c|c|c|c|c|}
\hline & & No Clone & Type I & Type II & Type III & Total \\
\hline \multirow{5}{*}{ Train } & Java & $2,496(67.22 \%)$ & $566(15.24 \%)$ & $277(7.46 \%)$ & $374(10.07 \%)$ & $3,713(100 \%)$ \\
\hline & JavaScript & $25,010(68.54 \%)$ & $5,425(14.87 \%)$ & $2,109(5.78 \%)$ & $3,945(10.81 \%)$ & $36,489(100 \%)$ \\
\hline & PHP & $1,582(63.89 \%)$ & $409(16.52 \%)$ & $137(5.53 \%)$ & $348(14.05 \%)$ & $2,476(100 \%)$ \\
\hline & Python & $7,025(76.01 \%)$ & $959(10.38 \%)$ & $371(4.01 \%)$ & $887(9.60 \%)$ & $9,242(100 \%)$ \\
\hline & Total & $36,113(69.56 \%)$ & $7,359(14.17 \%)$ & $2,894(5.57 \%)$ & $5,554(10.70 \%)$ & $51,920(100 \%)$ \\
\hline \multirow{5}{*}{ Test } & Java & $641(67.47 \%)$ & $157(16.53 \%)$ & $54(5.68 \%)$ & $98(10.32 \%)$ & $950(100 \%)$ \\
\hline & JavaScript & $6,243(68.94 \%)$ & $1,339(14.79 \%)$ & $514(5.68 \%)$ & $960(10.60 \%)$ & $9,056(100 \%)$ \\
\hline & PHP & $347(59.22 \%)$ & $113(19.28 \%)$ & $47(8.02 \%)$ & $79(13.48 \%)$ & $586(100 \%)$ \\
\hline & Python & $1,780(74.70 \%)$ & $264(11.08 \%)$ & $93(3.90 \%)$ & $246(10.32 \%)$ & $2,383(100 \%)$ \\
\hline & Total & $9,011(69.45 \%)$ & $1,873(14.44 \%)$ & $708(5.46 \%)$ & $1,383(10.66 \%)$ & $12,975(100 \%)$ \\
\hline
\end{tabular}

Table 4.7: The number of samples in the train and test sets for each label.

\begin{tabular}{|c|c|c|c|c|c|c|}
\hline & & Java & JavaScript & PHP & Python & Total \\
\hline \multirow{4}{*}{ Train } & One Clone Type & $431(35.41 \%)$ & $5,469(47.64 \%)$ & $258(28.86 \%)$ & $872(39.33 \%)$ & $7,030(44.47 \%)$ \\
\hline & Two Clone Types & $516(42.40 \%)$ & $3,892(33.91 \%)$ & $498(55.70 \%)$ & $874(39.42 \%)$ & $5,780(36.57 \%)$ \\
\hline & Three Clone Types & $270(22.19 \%)$ & $2,118(18.45 \%)$ & $138(15.44 \%)$ & $471(21.24 \%)$ & $2,997(18.96 \%)$ \\
\hline & Total & $1,217(100 \%)$ & $11,479(100 \%)$ & $894(100 \%)$ & $2,217(100 \%)$ & $15,807(100 \%)$ \\
\hline \multirow{4}{*}{ Test } & One Clone Type & $116(37.54 \%)$ & $1,319(46.89 \%)$ & $80(33.47 \%)$ & $253(41.96 \%)$ & $1,768(44.60 \%)$ \\
\hline & Two Clone Types & $148(47.90 \%)$ & $1,002(35.62 \%)$ & $108(45.19 \%)$ & $230(38.14 \%)$ & $1,488(37.54 \%)$ \\
\hline & Three Clone Types & $45(14.56 \%)$ & $492(17.49 \%)$ & $51(21.34 \%)$ & $120(19.90 \%)$ & $708(17.86 \%)$ \\
\hline & Total & $309(100 \%)$ & $2,813(100 \%)$ & $239(100 \%)$ & $603(100 \%)$ & $3,964(100 \%)$ \\
\hline
\end{tabular}

Table 4.8: Label distribution in the train and test sets. 


\begin{tabular}{ccccc}
\hline & Min & Max & Avg & Std Dev \\
\hline line & 1 & $22,051,108$ & 111,071 & $\pm 186,135.79$ \\
\hline commentLine & 0 & $1,077,519$ & 24,081 & $\pm 21,284.59$ \\
\hline keyword & 0 & $3,229,822$ & 62,188 & $\pm 81,812.78$ \\
\hline number & 0 & $1,008,100$ & 20,678 & $\pm 27,844.42$ \\
\hline name & 0 & $41,088,305$ & 123,719 & $\pm 799,593.20$ \\
\hline condition & 0 & 337,313 & 9,410 & $\pm 6,747.91$ \\
\hline loop & 0 & 13,312 & 1,764 & \pm 592.30 \\
\hline bracket & 0 & $44,378,124$ & 265,489 & $\pm 1,530,521.30$ \\
\hline punctuation & 0 & $44,551,152$ & 155,667 & $\pm 958,751.20$ \\
\hline operator & 0 & $5,935,101$ & 30,136 & $\pm 97,902.84$ \\
\hline comparator & 0 & $1,188,133$ & 7,500 & $\pm 5,952.00$ \\
\hline
\end{tabular}

Table 4.9: Range of statistical features.

\subsubsection{Feature Sets}

In this study, we extracted four feature sets based on programming paradigms, TFIDF approach, Code2Vec and Word2Vec models. The range of values for each numerical statistical feature in our dataset is shown in Table 4.9. In addition to these features, we had a categorical feature called "language", which we turned it to a one hot encoding vector. Except for "line", all other features may be absent in some samples. Section 3.3.4.1 provides a description of these features.

Table 4.10 shows the top 100 selected 1-gram to 3-gram words by TfidfVectorizer. The top five most frequent words in our dataset are this, function, var, if, and return. Because we only kept words with the length greater than one, most of the identifier names with one character have been eliminated. The goal of this task was to keep more meaningful words. 


\begin{tabular}{|c|c|c|c|c|c|c|c|c|c|}
\hline Rank & Word & Rank & Word & Rank & Word & Rank & Word & Rank & Word \\
\hline 1 & this & 21 & in & 41 & object & 61 & $\max$ & 81 & last \\
\hline 2 & function & 22 & set & 42 & url & 62 & string & 82 & number \\
\hline 3 & var & 23 & element & 43 & array & 63 & offset & 83 & left \\
\hline 4 & if & 24 & return this & 44 & math & 64 & function return & 84 & for var \\
\hline 5 & return & 25 & index & 45 & add & 65 & row & 85 & col \\
\hline 6 & is & 26 & export & 46 & at & 66 & day & 86 & month \\
\hline 7 & value & 27 & el & 47 & default & 67 & $\mathrm{~mm}$ & 87 & next \\
\hline 8 & else & 28 & typeof & 48 & callback & 68 & function var & 88 & child \\
\hline 9 & get & 29 & obj & 49 & result & 69 & error & 89 & format \\
\hline 10 & length & 30 & event & 50 & push & 70 & if this & 90 & $\min$ \\
\hline 11 & new & 31 & input & 51 & $\operatorname{tr}$ & 71 & argument & 91 & val \\
\hline 12 & data & 32 & node & 52 & start & 72 & class & 92 & style \\
\hline 13 & key & 33 & to & 53 & match & 73 & end & 93 & target \\
\hline 14 & null & 34 & case & 54 & opts & 74 & $\mathrm{fn}$ & 94 & state \\
\hline 15 & true & 35 & undefined & 55 & size & 75 & width & 95 & module export \\
\hline 16 & for & 36 & require & 56 & $\arg$ & 76 & config & 96 & map \\
\hline 17 & option & 37 & module & 57 & date & 77 & else if & 97 & out \\
\hline 18 & false & 38 & on & 58 & time & 78 & item & 98 & path \\
\hline 19 & name & 39 & self & 59 & parse & 79 & parent & 99 & at tr \\
\hline 20 & type & 40 & id & 60 & text & 80 & call & 100 & height \\
\hline
\end{tabular}

Table 4.10: Top 100 selected words by TfidfVectorizer. 


\begin{tabular}{ccccc}
\hline & \multicolumn{2}{c}{ Code2Vec } & \multicolumn{2}{c}{ Word2Vec } \\
\cline { 2 - 5 } & Train & Test & Train & Test \\
\hline Number of Not Identified Words & $1,986,087$ & 492,354 & $37,668,739$ & $9,126,039$ \\
\hline Total Number of Words & $1,712,005,545$ & $418,434,074$ & $1,712,005,545$ & $418,434,074$ \\
\hline Ratio \% & $0.12 \%$ & $0.12 \%$ & $2.20 \%$ & $2.18 \%$ \\
\hline
\end{tabular}

Table 4.11: Number of words that do not exist in the pre-trained models.

Here, we also conducted an experiment with pre-trained word embedding models that were used for feature extraction. We employed two pre-trained word embedding models, Code2Vec [99] and Word2Vec [100], which were pre-trained on the corpus related to software engineering. To determine how well they are fitted to our dataset, we looked at how many words in our dataset are not represented in the pre-trained models. Table 4.11 demonstrates that only $0.12 \%$ of words do not exist in the pretrained Code2Vec model, and 2.2\% in Word2Vec pre-trained model.

\subsubsection{Balancing}

Our goal is to determine how well clone types can be predicted. Since our dataset is unbalanced and most classification algorithms are less accurate with unbalanced data, we developed three separate balanced datasets. We have applied balancing only on the training set. Table 4.12 lists these train sets and the number of samples in each. The number of samples in the class "No Clone" is almost five times more than Type I, despite the fact that Type I has the greatest number of samples when compared to Types II and III. We now describe each balanced train set; we called them as "BalancedDS1", "BalancedDS2", and "BalancedDS3". For every balanced dataset, we applied the MLSMOTE technique to over-sample and randomly removed some samples in order to apply under-sampling.

- BalancedDS1. To obtain a nearly balanced dataset, we applied both undersampling and over-sampling techniques in this dataset. We over-sampled the 


\begin{tabular}{cccccc}
\hline \multirow{2}{*}{ Set } & \multirow{2}{*}{ Balancing } & \multicolumn{4}{c}{ Number of Samples } \\
\cline { 3 - 6 } & & Type I & Type II & Type III & No Clone \\
\hline OriginalDS & Imbalanced & 7,359 & 2,894 & 5,554 & 36,113 \\
\hline BalancedDS1 & MLSMOTE 10K + Under-sampling & 14,174 & 7,090 & 15,554 & 20,919 \\
\hline BalancedDS2 & MLSMOTE 20K + Under-sampling & 20,952 & 11,285 & 25,554 & 30,919 \\
\hline BalancedDS3 & Under-sampling & 7,359 & 2,894 & 5,554 & 10,919 \\
\hline
\end{tabular}

Table 4.12: Distribution of labels in the balanced train sets.

dataset by adding around 10,000 synthetic samples and under-sampled it by eliminating around 15,000 samples from the "No Clone" class. The results showed that after this balancing, the number of samples in the Type I class grew twofold, and the number of samples in the Type II and III classes increased about threefold.

- BalancedDS2. In this dataset, we used MLSMOTE and under-sampling again, but this time we added around 20,000 synthetic samples to the dataset and removed 10,000 samples at random from the majority class. In this dataset, we increased the number of synthetic samples when compared to BalancedDS1. So, the number of samples in Type I grew threefold over the original dataset, whereas Type II and III samples increased fivefold.

- BalancedDS3. In this dataset, just under-sampling was applied. The purpose in this case was to reduce the majority class samples while not adding synthetic data. We randomly removed around 20,000 samples from the "No Clone" class, which is the majority class, while the Type I to III classes remained unchanged. 


\subsubsection{RQ3: How Accurately Can We Predict the Clone Type Given Two Code Snippets?}

The results of models over our feature sets are discussed in this section. We ran eleven classifiers through each feature set in total. The objective is to identify the best classifier algorithm amongst the four multi-label classification models with Naive Bayes, Logistic Regression and Random Forest as the base classifiers for each model.

Table 4.13 shows the micro F1-Score and Hamming Loss of the eleven classifiers that were applied on the four datasets. The more detail results including Precision, Recall, Accuracy, and Execution Time are presented in the Table A.2, Table A.3. Table A.4, and Table A.5 for OriginalDS, BalancedDS1, BalancedDS2, and BalancedDS3, respectively. One of the observations from Table 4.13 for all feature sets and datasets is that top three models Random Forest with Binary Relevance, Label Powerset, and Label Space Partitioning Classifier have the best relative predictive performance, both in terms of micro F1-score and Hamming Loss; these classifiers have micro F1-Score more than $88 \%$ and Hamming Loss less than 6\%, among all the classifiers applied on all datasets and feature sets. Furthermore, no substantial difference in scores appears to exist between these three models in terms of different feature sets and datasets. Only the results for Statistical Features are slightly better than other feature sets.

In some models like mlknn, brknn, or Logistic Regression with Binary Relevance, Label Powerset, and Label Space Partitioning Classifier balancing not only did not improve the results, but it also reduced the amount of F1-Score. If we look at the F1-Score results for each class, which are shown in Table A.6, Table A.7, Table A.8, and Table A.9, we can notice that F1-Score in unbalanced data is high because of "No Clone" class. "No Clone" is a majority class and because of the great predictability of this class, the outcomes in these models are skewed higher for the OriginalDs. It 
means that balancing improved models' ability to predict minority classes, although reducing the overall F1-Score. For example the F1-Score for "Type I", "Type II", and "Type III" are $12.42 \%, 11.89 \%$, and $8.68 \%$ on the OriginalDS for $b r$-GNB model, while these results increased to $34.14 \%, 15.69 \%$, and $36.72 \%$ in the BalancedDS1. For "No Clone" class it decreased from $87.16 \%$ in the OriginalDS to $73.67 \%$ in the BalanacedDS1.

We retrieved three feature sets (Code2Vec, Word2Vec, and TF-IDF) using NLP techniques to compare their predictability in the field of code clone detection. However, we found no differences by comparing the model's results obtained from these feature sets. On the other hand, the results obtained from statistical features are slightly better than those features. The micro F1-Score in the OriginalDS for the best model ran over statistical features, which is Random Forest with Binary Relevance, is about $2 \%$ higher than the results of the best model's result over other feature sets. For the balanced datasets, this amount is approximately $1 \%$.

In terms of execution time, models based on the $G N B$ classifier had the quickest training time. For example, the $b r-G N B$ model took 4.6 seconds to train the OriginalDS, while $M L k N N$ took around 245.2 seconds. The F1-score of GNB models, on the other hand, is significantly lower than that of other models. We did not consider execution time when selecting the best model as we were aiming for a higher F1score. However, such performance consideration may become critical when deploying ML-based models.

Another experiment we conducted was to determine which features were the best predictors for the best classifier, Random Forest, and the best feature set, Statistical Features. We employed univariate feature selection in this experiment. It compares each feature to the target variable to identify whether they have a statistically significant relationship. It also ignores the other features while analyzing the link between one feature and the target variable. The features with the higher scores have a greater 


\begin{tabular}{|c|c|c|c|c|c|c|c|c|c|}
\hline \multirow[t]{2}{*}{ Feature Set } & \multirow[t]{2}{*}{ Model } & \multicolumn{2}{|c|}{ OriginalDS } & \multicolumn{2}{|c|}{ BalancedDS1 } & \multicolumn{2}{|c|}{ BalancedDS2 } & \multicolumn{2}{|c|}{ BalancedDS3 } \\
\hline & & F1-Score & Hamming loss & F1-Score & Hamming loss & F1-Score & Hamming loss & F1-Score & Hamming loss \\
\hline \multirow{11}{*}{ Code2Vec } & br_GNB & $47.89 \%$ & $36.49 \%$ & $49.04 \%$ & $30.91 \%$ & $46.06 \%$ & $33.56 \%$ & $46.95 \%$ & $36.72 \%$ \\
\hline & br LR & $73.74 \%$ & $13.69 \%$ & $59.85 \%$ & $20.06 \%$ & $58.75 \%$ & $21.40 \%$ & $60.01 \%$ & $18.61 \%$ \\
\hline & br_RF & $89.15 \%$ & $5.86 \%$ & $88.69 \%$ & $6.13 \%$ & $89.12 \%$ & $5.93 \%$ & $87.73 \%$ & $6.55 \%$ \\
\hline & lp_GNB & $50.19 \%$ & $30.50 \%$ & $38.50 \%$ & $40.78 \%$ & $34.72 \%$ & $44.94 \%$ & $47.83 \%$ & $32.24 \%$ \\
\hline & lp_LR & $74.92 \%$ & $13.26 \%$ & $71.05 \%$ & $15.91 \%$ & $70.15 \%$ & $16.53 \%$ & $71.70 \%$ & $15.27 \%$ \\
\hline & lp_RF & $89.08 \%$ & $5.93 \%$ & $88.79 \%$ & $6.15 \%$ & $89.07 \%$ & $6.00 \%$ & $88.47 \%$ & $6.32 \%$ \\
\hline & mlknn & $82.68 \%$ & $9.26 \%$ & $76.69 \%$ & $12.96 \%$ & $78.40 \%$ & $12.23 \%$ & $75.95 \%$ & $12.72 \%$ \\
\hline & brknn & $81.90 \%$ & $9.41 \%$ & $74.31 \%$ & $13.87 \%$ & $76.83 \%$ & $12.81 \%$ & $67.82 \%$ & $16.47 \%$ \\
\hline & lspc_GNB & $47.89 \%$ & $36.49 \%$ & $49.04 \%$ & $30.91 \%$ & $46.06 \%$ & $33.56 \%$ & $46.95 \%$ & $36.72 \%$ \\
\hline & lspc_LR & $73.74 \%$ & $13.69 \%$ & $59.85 \%$ & $20.06 \%$ & $58.75 \%$ & $21.40 \%$ & $60.01 \%$ & $18.61 \%$ \\
\hline & lspc_RF & $89.20 \%$ & $5.84 \%$ & $88.77 \%$ & $6.09 \%$ & $89.13 \%$ & $5.92 \%$ & $87.77 \%$ & $6.53 \%$ \\
\hline \multirow{11}{*}{ Word2Vec } & br_GNB & $43.54 \%$ & $41.29 \%$ & $43.84 \%$ & $38.99 \%$ & $43.05 \%$ & $38.09 \%$ & $43.17 \%$ & $41.13 \%$ \\
\hline & br_LR & $73.53 \%$ & $13.84 \%$ & $60.11 \%$ & $20.29 \%$ & $59.72 \%$ & $21.23 \%$ & $60.71 \%$ & $18.65 \%$ \\
\hline & br_RF & $89.33 \%$ & $5.77 \%$ & $88.69 \%$ & $6.13 \%$ & $89.04 \%$ & $5.97 \%$ & $87.52 \%$ & $6.65 \%$ \\
\hline & lp_GNB & $45.56 \%$ & $33.07 \%$ & $38.11 \%$ & $39.91 \%$ & $35.74 \%$ & $42.42 \%$ & $44.28 \%$ & $34.04 \%$ \\
\hline & lp_LR & $74.86 \%$ & $13.32 \%$ & $69.59 \%$ & $16.84 \%$ & $69.43 \%$ & $17.21 \%$ & $69.93 \%$ & $16.39 \%$ \\
\hline & lp_RF & $89.25 \%$ & $5.85 \%$ & $88.93 \%$ & $6.09 \%$ & $89.05 \%$ & $6.02 \%$ & $88.47 \%$ & $6.32 \%$ \\
\hline & mlknn & $82.89 \%$ & $9.03 \%$ & $78.74 \%$ & $12.16 \%$ & $78.42 \%$ & $12.20 \%$ & $76.55 \%$ & $12.32 \%$ \\
\hline & brknn & $82.05 \%$ & $9.32 \%$ & $74.43 \%$ & $13.80 \%$ & $76.75 \%$ & $12.84 \%$ & $68.41 \%$ & $16.24 \%$ \\
\hline & lspc_GNB & $43.54 \%$ & $41.29 \%$ & $43.84 \%$ & $38.99 \%$ & $43.05 \%$ & $38.09 \%$ & $43.17 \%$ & $41.13 \%$ \\
\hline & lspc_LR & $73.53 \%$ & $13.84 \%$ & $60.11 \%$ & $20.29 \%$ & $59.72 \%$ & $21.23 \%$ & $60.71 \%$ & $18.65 \%$ \\
\hline & lspc_RF & $89.29 \%$ & $5.79 \%$ & $88.65 \%$ & $6.15 \%$ & $89.00 \%$ & $5.98 \%$ & $87.44 \%$ & $6.70 \%$ \\
\hline \multirow{11}{*}{ TF-IDF } & br_GNB & $48.61 \%$ & $36.75 \%$ & $44.35 \%$ & $40.54 \%$ & $44.28 \%$ & $40.19 \%$ & $46.16 \%$ & $39.47 \%$ \\
\hline & br_LR & $73.20 \%$ & $13.93 \%$ & $60.19 \%$ & $19.99 \%$ & $59.38 \%$ & $21.13 \%$ & $61.14 \%$ & $18.26 \%$ \\
\hline & br_RF & $89.29 \%$ & $5.81 \%$ & $88.98 \%$ & $5.98 \%$ & $89.18 \%$ & $5.89 \%$ & $87.24 \%$ & $6.82 \%$ \\
\hline & lp_GNB & $30.98 \%$ & $45.11 \%$ & $27.09 \%$ & $48.01 \%$ & $26.95 \%$ & $48.19 \%$ & $29.73 \%$ & $46.12 \%$ \\
\hline & lp_LR & $73.95 \%$ & $13.74 \%$ & $71.50 \%$ & $15.58 \%$ & $69.97 \%$ & $16.76 \%$ & $71.27 \%$ & $15.43 \%$ \\
\hline & lp $\quad R F$ & $89.25 \%$ & $5.86 \%$ & $88.94 \%$ & $6.07 \%$ & $89.08 \%$ & $5.99 \%$ & $88.39 \%$ & $6.38 \%$ \\
\hline & mlknn & $82.71 \%$ & $9.25 \%$ & $76.79 \%$ & $13.04 \%$ & $78.51 \%$ & $12.23 \%$ & $73.34 \%$ & $13.96 \%$ \\
\hline & brknn & $81.93 \%$ & $9.43 \%$ & $75.04 \%$ & $13.46 \%$ & $77.39 \%$ & $12.43 \%$ & $69.52 \%$ & $15.63 \%$ \\
\hline & lspc_GNB & $48.61 \%$ & $36.75 \%$ & $44.35 \%$ & $40.54 \%$ & $44.28 \%$ & $40.19 \%$ & $46.16 \%$ & $39.47 \%$ \\
\hline & lspc_LR & $73.20 \%$ & $13.93 \%$ & $60.19 \%$ & $19.99 \%$ & $59.38 \%$ & $21.13 \%$ & $61.14 \%$ & $18.26 \%$ \\
\hline & lspc_RF & $89.26 \%$ & $5.82 \%$ & $88.87 \%$ & $6.04 \%$ & $89.18 \%$ & $5.89 \%$ & $87.30 \%$ & $6.79 \%$ \\
\hline \multirow{11}{*}{ Statistical } & br GNB & $68.44 \%$ & $18.18 \%$ & $52.83 \%$ & $37.16 \%$ & $52.50 \%$ & $37.38 \%$ & $53.04 \%$ & $36.51 \%$ \\
\hline & br_LR & $74.10 \%$ & $13.57 \%$ & $71.58 \%$ & $14.21 \%$ & $71.86 \%$ & $14.24 \%$ & $70.95 \%$ & $14.18 \%$ \\
\hline & br_RF & $91.18 \%$ & $4.76 \%$ & $90.04 \%$ & $5.39 \%$ & $90.68 \%$ & $5.06 \%$ & $88.58 \%$ & $6.05 \%$ \\
\hline & lp_GNB & $70.55 \%$ & $16.01 \%$ & $69.24 \%$ & $16.89 \%$ & $68.70 \%$ & $17.20 \%$ & $69.85 \%$ & $16.43 \%$ \\
\hline & lp_LR & $74.39 \%$ & $13.53 \%$ & $74.79 \%$ & $13.50 \%$ & $74.78 \%$ & $13.54 \%$ & $74.52 \%$ & $13.50 \%$ \\
\hline & lp_RF & $90.80 \%$ & $5.00 \%$ & $90.85 \%$ & $5.04 \%$ & $90.68 \%$ & $5.14 \%$ & $90.56 \%$ & $5.18 \%$ \\
\hline & mlknn & $84.40 \%$ & $8.24 \%$ & $80.53 \%$ & $10.86 \%$ & $81.41 \%$ & $10.43 \%$ & $80.29 \%$ & $10.64 \%$ \\
\hline & brknn & $84.40 \%$ & $8.24 \%$ & $81.47 \%$ & $9.97 \%$ & $82.63 \%$ & $9.45 \%$ & $78.41 \%$ & $11.15 \%$ \\
\hline & lspc_GNB & $68.44 \%$ & $18.18 \%$ & $52.83 \%$ & $37.16 \%$ & $52.50 \%$ & $37.38 \%$ & $53.04 \%$ & $36.51 \%$ \\
\hline & lspc_LR & $74.10 \%$ & $13.57 \%$ & $71.58 \%$ & $14.21 \%$ & $71.86 \%$ & $14.24 \%$ & $70.95 \%$ & $14.18 \%$ \\
\hline & lspc_RF & $91.07 \%$ & $4.80 \%$ & $90.13 \%$ & $5.34 \%$ & $90.75 \%$ & $5.02 \%$ & $88.72 \%$ & $5.97 \%$ \\
\hline
\end{tabular}

Table 4.13: Models' F1-score and hamming loss values for OriginalDB, BalancedDS1, BalancedDS2, and BalancedDS3. 


\begin{tabular}{cccccccccccccccc}
\hline & \multicolumn{1}{c}{ Binary Relevance } & \multicolumn{4}{c}{ Label Powerset } \\
\hline & Type I & Type II & Type III & No Clone & Type I & Type II & Type III & No Clone & Type I & Type II & Type III & No Clone \\
\hline Java & 7.14 & 32.40 & 0.51 & 0.64 & 7.14 & 32.40 & 0.51 & 0.64 & 7.14 & 32.40 & 0.51 & 0.64 \\
\hline JS & 45.00 & 8.76 & 1.04 & 105.80 & 45.00 & 8.76 & 1.04 & 105.80 & 45.00 & 8.76 & 1.04 & 105.80 \\
\hline PHP & 20.89 & 0.27 & 43.35 & 9.05 & 20.89 & 0.27 & 43.35 & 9.05 & 20.89 & 0.27 & 43.35 & 9.05 \\
\hline PY & 149.19 & 57.17 & 18.48 & 206.73 & 149.19 & 57.17 & 18.48 & 206.73 & 149.19 & 57.17 & 18.48 & 206.73 \\
\hline LineCNT & $1,357.50$ & 884.00 & $1,740.94$ & $2,270.73$ & $1,357.50$ & 884.00 & $1,740.94$ & $2,270.73$ & $1,357.50$ & 884.00 & $1,740.94$ & $2,270.73$ \\
\hline CommentLineCNT & 122.61 & 283.33 & 164.81 & 287.78 & 122.61 & 283.33 & 164.81 & 287.78 & 122.61 & 283.33 & 164.81 & 287.78 \\
\hline KeywordCNT & $1,637.64$ & $1,442.39$ & $1,185.87$ & $1,707.96$ & $1,637.64$ & $1,442.39$ & $1,185.87$ & $1,707.96$ & $1,637.64$ & $1,442.39$ & $1,185.87$ & $1,707.96$ \\
\hline NumberCNT & 1.36 & 1.63 & $1,257.31$ & 630.73 & 1.36 & 1.63 & $1,257.31$ & 630.73 & 1.36 & 1.63 & $1,257.31$ & 630.73 \\
\hline NameCNT & 181.95 & 223.22 & 654.27 & 508.31 & 181.95 & 223.22 & 654.27 & 508.31 & 181.95 & 223.22 & 654.27 & 508.31 \\
\hline ConditionCNT & 581.85 & 186.65 & 558.53 & $1,018.74$ & 581.85 & 186.65 & 558.53 & $1,018.74$ & 581.85 & 186.65 & 558.53 & $1,018.74$ \\
\hline LoopCNT & 279.68 & 544.54 & 585.74 & 745.94 & 279.68 & 544.54 & 585.74 & 745.94 & 279.68 & 544.54 & 585.74 & 745.94 \\
\hline BracketCNT & 240.28 & 148.70 & $1,937.59$ & $1,561.29$ & 240.28 & 148.70 & $1,937.59$ & $1,561.29$ & 240.28 & 148.70 & $1,937.59$ & $1,561.29$ \\
\hline PunctCNT & 284.99 & 117.60 & $1,036.36$ & $1,077.97$ & 284.99 & 117.60 & $1,036.36$ & $1,077.97$ & 284.99 & 117.60 & $1,036.36$ & $1,077.97$ \\
\hline OperatorCNT & 20.21 & 0.52 & 257.39 & 118.73 & 20.21 & 0.52 & 257.39 & 118.73 & 20.21 & 0.52 & 257.39 & 118.73 \\
\hline CompratorCNT & 60.85 & 68.79 & 142.00 & 134.03 & 60.85 & 68.79 & 142.00 & 134.03 & 60.85 & 68.79 & 142.00 & 134.03 \\
\hline
\end{tabular}

Table 4.14: F-score of statistical features for Random Forest based classifiers.

impact on classification quality. Table 4.14 shows the F-score of each label for three models, and demonstrates that while the number of lines and keywords are the two most effective features for all labels in our dataset, the number of brackets is beneficial in detecting Type III clones. However, all other features, such as the number of names, the condition, the loop, and the punctuation, are effective in predicting clone types. Language appears to be a weak predictor when compared to other features.

Answer to RQ3: We found that statistical features are stronger predictors than NLP-based features when predicting code clone types. On the other hand, while balancing has improved the performance of some classifiers in predicting the minority classes, multi-label classifiers that used Random Forest performed well even on the unbalanced data. We should note that the balancing techniques used in this study may not be effective for our dataset. 


\section{Chapter 5}

\section{Discussion}

In this chapter, we provide the general findings in Section 5.1, address some implications of this study in Section 5.2, and discuss potential threats to validity in Section 5.3 .

\section{$5.1 \quad$ Findings}

The primary objective of this study was to discover how developers reuse code snippets from online coding and programming forums such as Stack Overflow in their GitHub projects. The first part of the thesis dealt with detecting code clone types among Stack Overflow and GitHub code snippets. In more detail, we looked at the four well-known existing clone detection tools to identify which tool could detect clones between Stack Overflow and GitHub code pairs.

Stack Overflow code snippets are shorter than GitHub code snippets in the most cases. Additionally, Stack Overflow code snippets are often incomplete pieces of code intended to encompass the solution to a question. There are a number of restrictions in terms of choosing the right clone detection tool for this study. The biggest limitation

stems from the nature of Stack Overflow code snippets, which are mostly incomplete classes or methods. Hence, a detector must be able to parse code snippets that are 
not compilable or complete blocks. For example, SourcererCC and NiCad were not able to parse our code pairs because of this restriction. They support code detection at the level of method or function.

In addition, the majority of existing clone detectors were unable to support a variety of programming languages. Another barrier in this study was finding a tool that is able to detect clones of four different programming languages. NiCad, for example, was language-sensitive, and although supporting all programming languages in the dataset except JavaScript, we were unable to use it in our research due to the first limitation. SourcererCC, a scalable clone detector, just worked on Java code pairs in our dataset; and due to the first limitation it was not applicable for our study.

Furthermore, we needed to detect clone types, however, not all clone detectors provide such information. For example, CCFinder is a token-based clone detector and did work well on incomplete code and all programming languages in our dataset. However, it did not provide clone types. It discovered clones and calculated the similarity ratio of code pairs; this ratio was insufficient for our research questions. After all, ICLONES was the only tool that could detect the type of clones in our dataset for all programming languages.

ICLONES could not detect clones for the majority of the code pairs in our dataset. We can offer several explanations here. One of them could be the poor clone detection rate. However, we have performed a manual assessment and found it to be accurate in $90 \%$ of the cases. Even if we assume that the tool works properly and that all parameter settings are correct, the majority class (No Clone) remains relatively large in comparison to other classes. It indicates that developers are likely to incorporate that knowledge into projects without directly reusing code snippets from Stack Overflow. Or the Stack Overflow answer did not offer ready-to-use solutions or code, yet the discussion contained useful information (e.g., algorithms, pseudo code) that 
allowed developers to find a solution to their problem, so they added a reference to that Stack Overflow post to their code. Another scenario could be that a developer discovered a solution in one language (or even pseudo code) and then applied it to another language; as a result, no clones are detected between such code snippets. The limitation of the GHCodeSnippetHistory dataset can play a role here as well. Only accepted or top voted answers are linked to the GitHub snippets. Developers could potentially reuse alternative solutions that were offered within the comments of that discussion thread. In such cases, the approach of linking Stack Overflow code snippets to GitHub code snippets is not perfect and can yield false positives. Therefore, such cases can contribute to the detection of no clones as well.

Another explanation is related to the restriction of ICLONES in detecting Type IV clones which are defined as semantic clones. In our study, Type IV clones were not detected at all. Therefore, the "No Clone" class can potentially represent Type IV clones, at least partially. Since most of the tools that are capable of detecting Type IV clones are based on the machine learning techniques and using AST for feature extraction, we could not utilized them on our dataset.

According to the ICLONES detected clones, if developers reused Stack Overflow code snippets in their projects, they prefer to copy\&paste it with little to no modifications, as Type I was the most frequently discovered clone type with a $47.39 \%$ of detected clones. We also found that Type III was a common clone type. Type III clones accounted for approximately $35.01 \%$ of all detected clones. It demonstrates that around $35 \%$ of the Stack Overflow answers that offer code solutions are being modified by developers before they integrate them into their own projects. As the definition of Type III code clones indicates, this modification consists of adding or removing lines from a source code and using them in the target system. Type II had the least frequent clone type among the others, at a percentage of 17.61. This suggests that changes such as renaming identifiers occur less frequently by developers 
when reusing Stack Overflow code.

Another significant conclusion from the ICLONES results is that a code pair can contain multiple types of clones. When we detect multiple clone types for a pair, we speculate that developers are attempting to integrate some parts of code by modifying them while leaving other parts untouched.

The second part of the thesis focuses on predicting code clone types. We found that around 20,482 code pairs in our dataset had Type I, II, and/or III clones which was reduced to 19,771 after pre-processing. We had extracted four feature sets from the dataset. One of them was based on the frequency of programming paradigms in code pairs, while the other three were based on NLP approaches. The results revealed that statistical features based on programming paradigms were slightly better predictors for the dataset, while three others surprisingly had almost same results.

Code2Vec is a neural model that learns source code analogies. Although the model was trained on the Java code database, it can be applied to any codebase. Word2Vec is a Word2Vec model trained on Stack Overflow posts. The results of Code2Vec and Word2Vec were similar in our study. We speculate that both pre-trained models cover well the software engineering domain, so no difference is observed. Also, TF-IDF produced similar results to other extracted features. It is obvious that when we treat code as text, it is in fact less complex than natural language text such as in English, for example [87]. Texts in natural languages contain verbs, subjects, adverbs, and other sentence components, whereas code is mostly simple and repetitive [87. Furthermore, the variety of words in a program is fewer than that of a natural language text. While TF-IDF has the advantage of being a simple scoring scheme, word embeddings may be a preferable choice for most tasks where TF-IDF is utilized, especially when the task can benefit from the semantic similarity captured by word embeddings. However, in our study, both techniques produced the same results, demonstrating that in the case of code clone prediction, we can choose a less complex feature extraction method. 
In terms of balancing the dataset, because the "No Clone" class was nearly five times larger than the other classes, using MLSMOTE to augment the data alone could not be sufficient. Since this quantity of synthetic data has increased but the quality of data has declined. We applied MLSMOTE and under-sampling with different degree of augmentation and reduction. The findings of these experiments reveal that, while balancing improved prediction outcomes in minority classes in some of the evaluated models, it had little effect on the best models which used Random Forest classifier. MLSMOTE is designed to handle the class imbalance problem in multi-label datasets, however, we found that it does not perform well and thus, requires further improvement. According to our observations, it increases the data while maintaining the same quantity ratio among the labels as in the original dataset.

\subsection{Implications}

Our study have a wide range of applications and have the potential to accelerate development in other fields of research. Particularly in the prediction of clone types, which, to the best of our knowledge, have received no prior attention. This research is intended to have an impact on three main target audiences: 1) software developers and software companies, 2) researchers, and 3) online programming platforms.

\subsubsection{Software Developers}

Given the popularity of Stack Overflow and its influence on the software developers' community, the code reuse activity on Stack Overflow is definitely beneficial. On the other hand, low-quality, inaccurate, and/or malicious code shared on Stack Overflow may be reused by thousands of developers and make it into production software that may expose its users to security/data breaches [110]. According to the findings of this study, the most common code reuse was of Type I code clones, meaning that 
developers reused Stack Overflow code by copying and pasting it to their projects. One of the biggest problems with code reuse is that some developers reuse code snippets without thinking about what they are copying. The code may be outdated or may infringe a software license, include bugs, or expose security vulnerabilities. Therefore, to avoid such problems software developers must be extra cautious while relying on the ready-to-use code solutions from Stack Overflow.

While code reuse can be both beneficial and, sometimes, harmful, prediction of code clones can help with evaluating software system for the presence of specific clone types (e.g., Type I), thus mitigate the impact of high risk clones (e.g., security-related code snippets) and reduce overall software maintenance costs.

\subsubsection{Researchers}

Although many tools and strategies for detecting clones have been developed, this study showed that only a few of them are efficient at detecting clones on the code snippet level. With a rich source of online programming help being available to developers, they seek the knowledge and best practices shared by experts and rely on the ready-to-use solutions. Therefore, researchers should devote greater attention to this topic by developing better clone detection tools, detection of security vulnerabilities and malicious code snippets in online platforms [110], improving Stack Overflow's answer scoring system, and more. According to the findings of the second part of our research, detecting clones by leveraging ML-based approached is promising since they can be applied to different programming languages, code lengths (including Stack Overflow code snippets that are mostly short), and incomplete code blocks.

Various online Q\&A programming forums and developer communities exist nowadays, such as Stack Overflow, Stack Exchange [16], and CodeProject [111. In this study, we only focus on Stack Overflow. In order to build the body of knowledge on how developers reuse available online help, it would be necessary to conduct studies 
beyond Stack Overflow and investigate other online platforms to better understand the best practices of code reuse, as well as the pitfalls and obstacles that developers face when reusing code from different platforms.

\subsubsection{Online Programming Platforms}

It is beneficial for developer communities to encourage expert developers to share their expertise and knowledge on online programming platforms and promote knowledge transfer. However, online programming discussion platforms such as Stack Overflow should share responsibility in curating the content by automatically detecting and removing insecure code snippets [110, providing security related warnings to developers, or adding code snippet maturity/stability scores [2].

\subsection{Threats and Limitations}

Our study is subject to several threats to internal and external validity which we discuss next.

Internal Validity. To ensure the validity of the clone pairs we produced, we used a variety of approaches. First, we employed SourcererCC, NiCad, CCFinder, and ICLONES, four widely known clone detection tools. Due to their susceptibility to incomplete code snippets and detecting clone types, three of the clone detectors were unable to contribute to the study. As a result, we relied solely on ICLONES. We evaluated at the effects of different parameters for the ICLONES' minimum clone size and minimum block size and chose the most optimal ones. Nevertheless, false negatives, or code clones not recorded by the ICLONES, could potentially occur in our study. This limitation is related to the ICLONES' detection capability and power.

In this study, we focused on accepted Stack Overflow answers that solve the question's problem and are frequently displayed at the top of the answer list. However, 
we want to explore considering the top voted answers as future extension.

The clone type of 192 code pairs which were detected by the volunteer students is subject to manual judgement and human errors. Although we tried our best to be careful on searching for evidence and detecting the clones, some errors may still exist. We mitigated this bias by having two coders (participants) to detect the clones for the same code pair, as well as having the author of this study to verify their results. Additionally, all of the volunteers were Computer Science graduate and undergraduate students; the undergraduate students were in their third or fourth year of the bachelor's degree. However, we have not collected information regarding their programming experience and familiarity with different languages. This validation procedure can be further improved by engaging an investigator who is experienced with code clones. In addition, evaluating ICLONES's configuration settings based on the manual inspection results may decrease the accuracy of selecting the best parameters values.

We considered code snippets written in four most popular programming languages. We relied on the programming language tag on the Stack Overflow code snippet and the extension of its correlated GitHub commit. However, it is possible that code injection existed in some posts or commits, especially for a web-based programming language like PHP. We have considered the programming language as one of the features in our statistical feature set. Therefore, the sample might be misclassified and affect the models' performance. In addition, we summed up the calculated features from Stack Overflow and GitHub code snippets in the statistical features section. We did not test the models' results using subtraction rather than sum. Tangled commits is another topic that might have an impact on extracted feature sets and model accuracy. Tangled commits blend different concerns, whereas a contribution to a code repository should ideally focus on a single concern. In practice, however, many commits tangle problems due to time constraints or a concern's unclear boundaries. Thus, the accuracy of classification models may be harmed by tangled commits [112]. 
External Validity. We carefully selected the dataset for our study so that the results might be applied to a wide range of applications. However, we relied on the top four programming languages from the Stack Overflow which is the most popular programming Q\&A website. So, the findings of this study may not be representative to all the programming languages and Q\&A websites.

In terms of Stack Overflow code snippets, we downloaded a full data dump and extracted accepted answers or top voted answer (if no accepted answer is available), as they are the ones that are most likely to be reused. In the SOTorrent dataset, each Stack Overflow code block has different versions. When a code block is updated, a new version is created. To determine which version is utilized in the GitHub project, we select a code block with the creation date that is posted prior to the corresponding GitHub commit's date. However, this matching may not be accurate as earlier versions of the code block may have been reused in GitHub. When comparing different versions of a code block on Stack Overflow, the type of clone detected may be affected. Our findings are constrained by the data collection process [2]. They may not be generalizable to all Stack Overflow answers.

Furthermore, we have relied on the collected GitHub commits in the Manes and Baysal [2] study. The concerns to validity addressed in their work (e.g., the accuracy of traceability between Stack Overflow and GitHub) may have an impact on our study as well. 


\section{Chapter 6}

\section{Conclusions}

Lastly, the thesis concludes with a summary of our work and contributions in Section 6.1, and then discusses future directions in Section 6.2

\subsection{Summary of Contributions}

Most software developers prefer to copy-paste program code rather than writing new code from scratch. This reuse of code is referred to as "code cloning" in a software system. Popular Q\&A forums like Stack Overflow include a plethora of reusable code snippets. This research looked into how software developers reuse code snippets from Stack Overflow in the projects hosted on GitHub, as well as how much those reused code snippets are modified by the developers. We have created a code pair dataset that maps Stack Overflow code snippets to GitHub commits with the help of SOTorrent and GHCodeSnippetHistory. We concentrated on identifying different types of clones between Stack Overflow and GitHub code snippets in the first part of the study. In the second part, we developed and tested multi-label classifiers for predicting the types of code clones.

Detecting Code Clones. Our preliminary findings show that GitHub developers adopt different practices when reusing Stack Overflow code snippets in their projects. 
We found that only one of four well-known clone detector tools, ICLONES, can detect clones for non-complete blocks of code and various programming languages. Based on our findings, the programming language has no effect on the accuracy of ICLONES. To configure and assess ICLONES, we compared the results of ICLONES with the results of the manual detection of clones by human subjects. We also observed that around $76.9 \%$ of the code pairs in our dataset did not have any clones detected. This implies that developers do refer to Stack Overflow discussions (since our GitHub snippets include references to Stack Overflow posts as per Stack Overflow's license model [113]), but they rather adopt and integrate online help into their projects. We can also argue that this large percentage of snippets with no detected clones is rather inflated as ICLONES is only capable of detecting three types of clones; the existing state-of-theart techniques are unable to detect Type IV (syntactically different) clones on the snippet level.

On the other hand, among the code pairs having clones, Type I (15.85\%) and Type III (11.71\%) are the most frequently detected clones between Stack Overflow and GitHub code snippets, while Type II (5.89\%) is a less common type of clones. This demonstrates that if developers do reuse code from Stack Overflow, they copy-paste snippets more often than they adopt them to their GitHub projects. Additionally, we observed that some code pairs contained more than one type of clones meaning that developers may modify some blocks of the reused code snippet but keep other parts as is.

Predicting Code Clone Types. We tried to find a model for predicting code clone types between Stack Overflow code snippets and its associated GitHub commits in this study. We had two objectives in mind - to determine: 1) which extracted feature set is a better predictor, and 2) which classifier is a better learner to predict clone types. We treated code samples as natural texts and used NLP techniques to extract features from them. We used TF-IDF, Code2Vec, and Word2Vec models to 
extract features in addition to the statistical feature set derived from the programming paradigms. The results of applying classifiers to our extracted feature sets revealed that most of our models are likely to overfit for the majority class, and there is no significant difference in detecting clone types between the best models or even between feature sets. However, statistical feature set was slightly better than others. Also, even on unbalanced dataset, models that used Random Forest as a basic classifier produced more accurate results.

\subsection{Future Work}

There are a number of research opportunities that can be built on top of this work. In this study, we focused on detecting code clones among two platforms. The findings of this study can be extended by detecting clone types among Stack Overflow code blocks' versions and GitHub commits, and also different linkage to the alternative solutions on the Stack Overflow. In addition, other online Q\&A programming platforms can be utilized to gain a more generalized understanding of developers' behaviour while reusing online code.

According to the findings of this study, approximately $77 \%$ of code pairs did not contain clones. Despite the fact that we explained why so many of our code pairings lacked clones, a possible future direction can be a qualitative study to understand the reasons of why developers refer to Stack Overflow posts. Such reasons could be due to cross-language usage, inspiration, or other considerations.

In this work, we also select specific commits from GitHub that have references to Stack Overflow posts. In other words, we focused on code snippet pairs. We did not consider code reuse at a project level. It can be interesting to explore how different types of clones are distributed in different kinds of projects. Currently, we worked on the language level, but we can expend our work to the type of application such as their 
category, size, or domain. Future studies can investigate whether the distribution of clone types differs for small, mid- or large-sized projects, or to what extend ML-based projects (e.g., Jupyter notebooks) experience a similar ratio of code clones compared to application systems. Another interesting direction would be to study which clone type is more error prone or more likely to introduce bugs to a project.

One possible application of this work can be for educational purposes. Students often refer to online programming help and reuse codes from such platforms in their projects. With a prediction model we can predict plagiarism in their code by knowing the trends of likely clone types to be present in their assignments or projects.

For our prediction model, we relied on the standard multi-label classification techniques, but we can extend them to deep learning approaches as well. Deep learning approaches can be applied for feature extraction as well as classification. 


\section{List of References}

[1] T. S. Dataset, "https://empirical-software.engineering/sotorrent/." (accessed: 2021-07-12).

[2] S. S. Manes and O. Baysal, "Studying the Change Histories of Stack Overflow and GitHub Snippets," pp. 283-294, 2021.

[3] G. Mostaeen, B. Roy, C. K. Roy, K. Schneider, and J. Svajlenko, "A machine learning based framework for code clone validation," Journal of Systems and Software, vol. 169, p. 110686, nov 2020.

[4] C. Kapser and M. W. Godfrey, "Cloning considered harmful" considered harmful," Proceedings - Working Conference on Reverse Engineering, WCRE, pp. 19$28,2006$.

[5] D. M. German, M. Di Penta, Y. G. Guéhéneuc, and G. Antoniol, "Code siblings: Technical and legal implications of copying code between applications," Proceedings of the 2009 6th IEEE International Working Conference on Mining Software Repositories, MSR 2009, pp. 81-90, 2009.

[6] StackOverflow, "Stack Overflow - Where Developers Learn, Share, \& Build Careers."

[7] StackOverflow, "https://insights.stackoverflow.com/survey/2020." (accessed: 2021-06-16).

[8] GitHub, "https://github.com/." (accessed: 2021-07-09).

[9] S. S. Manes, Studying the Change History of Code Snippets on Stack Overflow and GitHub [MSc thesis]. No. May, 2020.

[10] C. Ragkhitwetsagul, J. Krinke, M. Paixao, G. Bianco, and R. Oliveto, "Toxic Code Snippets on Stack Overflow," IEEE Transactions on Software Engineering, vol. 47, pp. 560-581, mar 2021. 
[11] C. K. Roy and J. R. Cordy, "A Survey on Software Clone Detection Research," tech. rep., No. 2007-541, School of Computing, Queen's University, Canada, 2007.

[12] W. Bai, O. Akgul, and M. L. Mazurek, "A Qualitative Investigation of Insecure Code Propagation from Online Forums," in 2019 IEEE Cybersecurity Development (SecDev), pp. 34-48, IEEE, sep 2019.

[13] D. Yang, P. Martins, V. Saini, and C. Lopes, "Stack Overflow in Github: Any Snippets There?," in 2017 IEEE/ACM 14th International Conference on Mining Software Repositories (MSR), pp. 280-290, IEEE, may 2017.

[14] T. Zhang, D. Yang, C. Lopes, and M. Kim, "Analyzing and Supporting Adaptation of Online Code Examples," in 2019 IEEE/ACM 41st International Conference on Software Engineering (ICSE), vol. 2019-May, pp. 316-327, IEEE, may 2019.

[15] S. Baltes, L. Dumani, C. Treude, and S. Diehl, "SOTorrent: Reconstructing and Analyzing the Evolution of Stack Overflow Posts," in Proceedings of the 15th International Conference on Mining Software Repositories, (New York, NY, USA), pp. 319-330, ACM, may 2018.

[16] StackExchange, "Stack Exchange Data Dump : Stack Exchange, Inc. : Free Download, Borrow, and Streaming : Internet Archive."

[17] C. J. Kapser, Toward an Understanding of Software Code Cloning as a Development Practice by. PhD thesis, Waterloo, 2009.

[18] B. M. Lange and T. G. Moher, "Some strategies of reuse in an object-oriented programming environment," ACM SIGCHI Bulletin, vol. 20, pp. 69-73, mar 1989.

[19] C. W. Krueger, "Software reuse," ACM Computing Surveys, vol. 24, pp. 131183, jun 1992 .

[20] R. Prieto-Diaz, "Status report: software reusability," IEEE Software, vol. 10, pp. 61-66, may 1993.

[21] M. B. Rosson and J. M. Carroll, "Active Programming Strategies in Reuse," in ECOOP' 93 - Object-Oriented Programming, pp. 4-20, Berlin, Heidelberg: Springer Berlin Heidelberg, 1993.

[22] R. Holmes, Pragmatic software reuse. PhD thesis, CALGARY, 2008. 
[23] R. E. Johnson and B. Foote, "Designing Reuseable Classes," Object-Oriented Programming, vol. 1, no. 2, pp. 22-35, 1988.

[24] B. Meyer, "On to components," Computer, vol. 32, no. 1, pp. 139-143, 1999.

[25] M. Mezini and K. Ostermann, "Integrating independent components with ondemand remodularization," ACM SIGPLAN Notices, vol. 37, pp. 52-67, nov 2002.

[26] J. Parsons and C. Saunders, "Cognitive heuristics in software engineering applying and extending anchoring and adjustment to artifact reuse," IEEE Transactions on Software Engineering, vol. 30, pp. 873-888, dec 2004.

[27] J. Krinke, "Identifying similar code with program dependence graphs," in Proceedings Eighth Working Conference on Reverse Engineering, pp. 301-309, IEEE Comput. Soc, 2001.

[28] T. A. Standish, "An Essay on Software Reuse," IEEE Transactions on Software Engineering, vol. SE-10, pp. 494-497, sep 1984.

[29] H. H. Thompson and J. A. Whittaker, "The Business Case for Software Security," Dr. Dobb's Journal, vol. 29, no. 3, pp. 64-66, 2004.

[30] B. Schell and M. Clemens, Webster's New world Hackers dictionary. 2006.

[31] R. Koschke, R. Falke, and P. Frenzel, "Clone Detection Using Abstract Syntax Suffix Trees," in 2006 13th Working Conference on Reverse Engineering, pp. 253-262, IEEE, 2006.

[32] S. Ducasse, M. Rieger, and S. Demeyer, "A language independent approach for detecting duplicated code," in Proceedings IEEE International Conference on Software Maintenance - 1999 (ICSM'99). 'Software Maintenance for Business Change' (Cat. No.99CB36360), pp. 109-118, IEEE, 1999.

[33] R. Komondoor and S. Horwitz, "Using Slicing to Identify Duplication in Source Code," in International Symposium on Static Analysis, pp. 40-56, 2001.

[34] N. Davey, P. Barson, S. Field, and R. J. Frank, "The Development of a Software Clone Detector," International Journal of Applied Software Technology, vol. 1, no. 3-4, pp. 219-236, 1995.

[35] G. Mishne, M. De Rijke, and M. Marx, "Source code retrieval using conceptual graphs," Proceedings RIAO, no. December, pp. 539-554, 2004. 
[36] Q. U. Ain, W. H. Butt, M. W. Anwar, F. Azam, and B. Maqbool, "A Systematic Review on Code Clone Detection," IEEE Access, vol. 7, pp. 86121-86144, 2019.

[37] T. Vislavski, G. Rakic, N. Cardozo, and Z. Budimac, "LICCA: A tool for cross-language clone detection," in 2018 IEEE 25th International Conference on Software Analysis, Evolution and Reengineering (SANER), vol. 2018-March, pp. 512-516, IEEE, mar 2018.

[38] N. Saini, S. Singh, and Suman, "Code Clones: Detection and Management," Procedia Computer Science, vol. 132, pp. 718-727, 2018.

[39] T. Kamiya, S. Kusumoto, and K. Inoue, "CCFinder: a multilinguistic tokenbased code clone detection system for large scale source code," IEEE Transactions on Software Engineering, vol. 28, pp. 654-670, jul 2002.

[40] H. A. Basit and S. Jarzabek, "Detecting higher-level similarity patterns in programs," ACM SIGSOFT Software Engineering Notes, vol. 30, pp. 156-165, sep 2005 .

[41] Z. Li, S. Lu, S. Myagmar, and Y. Zhou, "CP-Miner: finding copy-paste and related bugs in large-scale software code," IEEE Transactions on Software Engineering, vol. 32, pp. 176-192, mar 2006.

[42] L. Jiang, G. Misherghi, Z. Su, and S. Glondu, "DECKARD: Scalable and Accurate Tree-Based Detection of Code Clones," in 29th International Conference on Software Engineering (ICSE'07), no. 0520320, pp. 96-105, IEEE, may 2007.

[43] C. Roy and J. Cordy, "NICAD: Accurate Detection of Near-Miss Intentional Clones Using Flexible Pretty-Printing and Code Normalization," in 2008 16th IEEE International Conference on Program Comprehension, pp. 172-181, IEEE, jun 2008.

[44] N. Göde and R. Koschke, "Incremental Clone Detection," in 2009 13th European Conference on Software Maintenance and Reengineering, pp. 219-228, IEEE, 2009 .

[45] M. S. Uddin, C. K. Roy, K. A. Schneider, and A. Hindle, "On the Effectiveness of Simhash for Detecting Near-Miss Clones in Large Scale Software Systems," in 2011 18th Working Conference on Reverse Engineering, pp. 13-22, IEEE, oct 2011. 
[46] R. Koschke, "Large-scale inter-system clone detection using suffix trees and hashing," Journal of Software: Evolution and Process, vol. 26, pp. 747-769, aug 2014 .

[47] E. Kodhai and S. Kanmani, "Method-level code clone detection through LWH (Light Weight Hybrid) approach," Journal of Software Engineering Research and Development, vol. 2, p. 12, dec 2014.

[48] M. White, M. Tufano, C. Vendome, and D. Poshyvanyk, "Deep learning code fragments for code clone detection," in Proceedings of the 31st IEEE/ACM International Conference on Automated Software Engineering, (New York, NY, USA), pp. 87-98, ACM, aug 2016.

[49] H. Sajnani, V. Saini, J. Svajlenko, C. K. Roy, and C. V. Lopes, "SourcererCC," in Proceedings of the 38th International Conference on Software Engineering, vol. 14-22-May-, (New York, NY, USA), pp. 1157-1168, ACM, may 2016.

[50] L. Li, H. Feng, W. Zhuang, N. Meng, and B. Ryder, "CCLearner: A Deep Learning-Based Clone Detection Approach," in 2017 IEEE International Conference on Software Maintenance and Evolution (ICSME), pp. 249-260, IEEE, sep 2017.

[51] H. Wei and M. Li, "Supervised Deep Features for Software Functional Clone Detection by Exploiting Lexical and Syntactical Information in Source Code," in Proceedings of the Twenty-Sixth International Joint Conference on Artificial Intelligence, vol. 0, (California), pp. 3034-3040, International Joint Conferences on Artificial Intelligence Organization, aug 2017.

[52] S. Kim, S. Woo, H. Lee, and H. Oh, "VUDDY: A Scalable Approach for Vulnerable Code Clone Discovery," in 2017 IEEE Symposium on Security and Privacy (SP), pp. 595-614, IEEE, may 2017.

[53] V. Saini, F. Farmahinifarahani, Y. Lu, P. Baldi, and C. V. Lopes, "Oreo: detection of clones in the twilight zone," in Proceedings of the 2018 26th ACM Joint Meeting on European Software Engineering Conference and Symposium on the Foundations of Software Engineering, (New York, NY, USA), pp. 354365, ACM, oct 2018.

[54] G. Zhao and J. Huang, "DeepSim: deep learning code functional similarity," in Proceedings of the 2018 26th ACM Joint Meeting on European Software Engineering Conference and Symposium on the Foundations of Software Engineering, (New York, NY, USA), pp. 141-151, ACM, oct 2018. 
[55] H. Yu, W. Lam, L. Chen, G. Li, T. Xie, and Q. Wang, "Neural Detection of Semantic Code Clones Via Tree-Based Convolution," in 2019 IEEE/ACM 27th International Conference on Program Comprehension (ICPC), vol. 2019-May, pp. 70-80, IEEE, may 2019.

[56] K. W. Nafi, T. S. Kar, B. Roy, C. K. Roy, and K. A. Schneider, "CLCDSA: Cross Language Code Clone Detection using Syntactical Features and API Documentation," in 2019 34th IEEE/ACM International Conference on Automated Software Engineering (ASE), pp. 1026-1037, IEEE, nov 2019.

[57] J. Zhang, X. Wang, H. Zhang, H. Sun, K. Wang, and X. Liu, "A Novel Neural Source Code Representation Based on Abstract Syntax Tree," in 2019 IEEE/ACM 41st International Conference on Software Engineering (ICSE), vol. 2019-May, pp. 783-794, IEEE, may 2019.

[58] CCFinder, "http://www.ccfinder.net/ccfinderxos.html." (accessed: 2021-07$03)$.

[59] Y. Semura, N. Yoshida, E. Choi, and K. Inoue, "CCFinderSW: Clone Detection Tool with Flexible Multilingual Tokenization," in 2017 24th Asia-Pacific Software Engineering Conference (APSEC), vol. 2017-Decem, pp. 654-659, IEEE, $\operatorname{dec} 2017$.

[60] A. C. P. L. F. de Carvalho and A. A. Freitas, "A Tutorial on Multi-label Classification Techniques," in Studies in Computational Intelligence, vol. 205, pp. 177195, 2009.

[61] G. James, D. Witten, T. Hastie, and R. Tibshirani, "Linear Regression," in Current medicinal chemistry, vol. 7, pp. 59-126, 2013.

[62] G. James, D. Witten, T. Hastie, and R. Tibshirani, "Support Vector Machines," in Current Medicinal Chemistry, vol. 7, pp. 337-372, 2013.

[63] M. Crawford, T. M. Khoshgoftaar, J. D. Prusa, A. N. Richter, and H. Al Najada, "Survey of review spam detection using machine learning techniques," Journal of Big Data, vol. 2, p. 23, dec 2015.

[64] M. L. Gatza, J. E. Lucas, W. T. Barry, J. W. Kim, Q. Wang, M. D. Crawford, M. B. Datto, M. Kelley, B. Mathey-Prevot, A. Potti, and J. R. Nevins, "A pathway-based classification of human breast cancer," Proceedings of the National Academy of Sciences, vol. 107, pp. 6994-6999, apr 2010. 
[65] C. A. Escobar and R. Morales-Menendez, "Machine learning techniques for quality control in high conformance manufacturing environment," Advances in Mechanical Engineering, vol. 10, p. 168781401875551, feb 2018.

[66] B. P. Chacko, V. R. Vimal Krishnan, G. Raju, and P. Babu Anto, "Handwritten character recognition using wavelet energy and extreme learning machine," International Journal of Machine Learning and Cybernetics, vol. 3, pp. 149-161, jun 2012.

[67] Y. Taigman, M. Yang, M. Ranzato, and L. Wolf, "DeepFace: Closing the Gap to Human-Level Performance in Face Verification," in 2014 IEEE Conference on Computer Vision and Pattern Recognition, pp. 1701-1708, IEEE, jun 2014.

[68] Meng Joo Er, R. Venkatesan, and Ning Wang, "An online universal classifier for binary, multi-class and multi-label classification," in 2016 IEEE International Conference on Systems, Man, and Cybernetics (SMC), pp. 003701003706, IEEE, oct 2016.

[69] J. Wehrmann and R. C. Barros, "Movie genre classification: A multi-label approach based on convolutions through time," Applied Soft Computing, vol. 61, pp. 973-982, dec 2017.

[70] D. Xu, Y. Shi, I. W. Tsang, Y.-S. Ong, C. Gong, and X. Shen, "Survey on Multi-Output Learning," IEEE Transactions on Neural Networks and Learning Systems, vol. 31, no. 7, pp. 1-21, 2019.

[71] W. JUNG, E. LEE, and C. WU, "A Survey on Mining Software Repositories," IEICE Transactions on Information and Systems, vol. E95.D, no. 5, pp. 13841406, 2012.

[72] S. Agarwal, "Data Mining: Data Mining Concepts and Techniques," in 2013 International Conference on Machine Intelligence and Research Advancement, pp. 203-207, IEEE, dec 2013.

[73] MSR, "http://www.msrconf.org." (accessed: 2021-07-06).

[74] E. Moritz, M. Linares-Vasquez, D. Poshyvanyk, M. Grechanik, C. McMillan, and M. Gethers, "ExPort: Detecting and visualizing API usages in large source code repositories," in 2013 28th IEEE/ACM International Conference on Automated Software Engineering (ASE), pp. 646-651, IEEE, nov 2013.

[75] S.-K. Hsu and S.-J. Lin, "MACs: Mining API code snippets for code reuse," Expert Systems with Applications, vol. 38, pp. 7291-7301, jun 2011. 
[76] Jonathan Chang and David Blei, "Relational Topic Models for Document Networks," Ceskoslovenska gynekologie, vol. 18, pp. 45-9, jan 1953.

[77] S. K. Kuttal, A. Sarma, and G. Rothermel, "History repeats itself more easily when you log it: Versioning for mashups," in 2011 IEEE Symposium on Visual Languages and Human-Centric Computing (VL/HCC), pp. 69-72, IEEE, sep 2011.

[78] Bugzilla, "https://www.bugzilla.org/." (accessed: 2021-07-06).

[79] Y. Yan, M. Menarini, and W. Griswold, "Mining Software Contracts for Software Evolution," in 2014 IEEE International Conference on Software Maintenance and Evolution, pp. 471-475, IEEE, sep 2014.

[80] A. Lotter, S. A. Licorish, B. T. R. Savarimuthu, and S. Meldrum, "Code Reuse in Stack Overflow and Popular Open Source Java Projects," in 2018 25th Australasian Software Engineering Conference (ASWEC), pp. 141-150, IEEE, nov 2018.

[81] H. Gonzalez, N. Stakhanova, and A. A. Ghorbani, "Measuring code reuse in Android apps," in 201614 th Annual Conference on Privacy, Security and Trust (PST), pp. 187-195, IEEE, dec 2016.

[82] A. Ciborowska, N. A. Kraft, and K. Damevski, "Detecting and characterizing developer behavior following opportunistic reuse of code snippets from the web," in Proceedings of the 15th International Conference on Mining Software Repositories, (New York, NY, USA), pp. 94-97, ACM, may 2018.

[83] G. Digkas, N. Nikolaidis, A. Ampatzoglou, and A. Chatzigeorgiou, "Reusing Code from StackOverflow: The Effect on Technical Debt," in 2019 45th Euromicro Conference on Software Engineering and Advanced Applications (SEAA), pp. 87-91, IEEE, aug 2019.

[84] S. Baltes, R. Kiefer, and S. Diehl, "Attribution Required: Stack Overflow Code Snippets in GitHub Projects," in 2017 IEEE/ACM 39th International Conference on Software Engineering Companion (ICSE-C), pp. 161-163, IEEE, may 2017.

[85] R. Abdalkareem, E. Shihab, and J. Rilling, "On code reuse from StackOverflow: An exploratory study on Android apps," Information and Software Technology, vol. 88, pp. 148-158, aug 2017. 
[86] M. A. Nishi, A. Ciborowska, and K. Damevski, "Characterizing Duplicate Code Snippets between Stack Overflow and Tutorials," in 2019 IEEE/ACM 16th International Conference on Mining Software Repositories (MSR), vol. 2019-May, pp. 240-244, IEEE, may 2019.

[87] A. Hindle, E. T. Barr, Z. Su, M. Gabel, and P. Devanbu, "On the naturalness of software," in 2012 34th International Conference on Software Engineering (ICSE), pp. 837-847, IEEE, jun 2012.

[88] Y. Zhang, W. Zheng, and M. Li, "Learning Uniform Semantic Features for Natural Language and Programming Language Globally, Locally and Sequentially," Proceedings of the AAAI Conference on Artificial Intelligence, vol. 33, pp. 5845-5852, jul 2019.

[89] S. Sachdev, H. Li, S. Luan, S. Kim, K. Sen, and S. Chandra, "Retrieval on source code: a neural code search," in Proceedings of the 2nd ACM SIGPLAN International Workshop on Machine Learning and Programming Languages, (New York, NY, USA), pp. 31-41, ACM, jun 2018.

[90] K. Alrashedy, D. Dharmaretnam, D. M. German, V. Srinivasan, and T. Aaron Gulliver, $S C C++$ : Predicting the programming language of questions and snippets of Stack Overflow, vol. 162. apr 2020.

[91] M. Allamanis, E. T. Barr, C. Bird, and C. Sutton, "Learning natural coding conventions," in Proceedings of the 22nd ACM SIGSOFT International Symposium on Foundations of Software Engineering, (New York, NY, USA), pp. 281-293, ACM, nov 2014.

[92] BigQuery, "https://cloud.google.com/bigquery." (accessed: 2021-07-09).

[93] G. Gousios, "The GHTorent dataset and tool suite," in 2013 10th Working Conference on Mining Software Repositories (MSR), pp. 233-236, IEEE, may 2013.

[94] J. Cohen, "A coefficient of agreement for nominal scale," no. 1, pp. 37-46, 1960.

[95] J. Harder and N. Göde, "Efficiently handling clone data," in Proceeding of the 5th international workshop on Software clones - IWSC '11, (New York, New York, USA), p. 81, ACM Press, 2011.

[96] S. Bird, E. Loper, and K. Ewan, Natural Language Processing with Python. O’Reilly Media Inc, 2009. 
[97] WordNinja, "https://github.com/keredson/wordninja." (accessed: 2021-07-20).

[98] T. Mikolov, K. Chen, G. Corrado, and J. Dean, "Efficient Estimation of Word Representations in Vector Space," 1st International Conference on Learning Representations, ICLR 2013 - Workshop Track Proceedings, pp. 1-12, jan 2013.

[99] U. Alon, M. Zilberstein, O. Levy, and E. Yahav, "code2vec: learning distributed representations of code," Proceedings of the ACM on Programming Languages, vol. 3, pp. 1-29, jan 2019.

[100] V. Efstathiou, C. Chatzilenas, and D. Spinellis, "Word embeddings for the software engineering domain," in Proceedings of the 15th International Conference on Mining Software Repositories, (New York, NY, USA), pp. 38-41, ACM, may 2018.

[101] M. Sorower, "A literature survey on algorithms for multi-label learning," Oregon State University, Corvallis, pp. 1-25, 2010.

[102] G. Tsoumakas and I. Vlahavas, "Random k-Labelsets: An Ensemble Method for Multilabel Classification," in Machine Learning: ECML 200\%, vol. 4701 LNAI, pp. 406-417, Berlin, Heidelberg: Springer Berlin Heidelberg, 2007.

[103] M.-l. Zhang and Z.-h. Zhou, "Ml-knnA Lazy Learning Approach to.pdf," Pattern recognition, vol. 40, no. 7, pp. 2038-2048, 2007.

[104] E. Spyromitros, G. Tsoumakas, and I. Vlahavas, "An Empirical Study of Lazy Multilabel Classification Algorithms," in roc. 5th Hellenic Conference on Artificial Intelligence (SETN 2008), (Syros, Greece), pp. 401-406, 2008.

[105] P. Szymański, T. Kajdanowicz, and K. Kersting, "How Is a Data-Driven Approach Better than Random Choice in Label Space Division for Multi-Label Classification?," Entropy, vol. 18, p. 282, jul 2016.

[106] P. Flach, "Machine Learning: The Art and Science of Algorithms That Make Sense of Data," 2012.

[107] F. Charte, A. J. Rivera, M. J. Del Jesus, and F. Herrera, "MLSMOTE: Approaching imbalanced multilabel learning through synthetic instance generation," Knowledge-Based Systems, vol. 89, pp. 385-397, 2015.

[108] N. V. Chawla, K. W. Bowyer, L. O. Hall, and W. P. Kegelmeyer, "SMOTE: Synthetic Minority Over-sampling Technique," Journal of Artificial Intelligence Research, vol. 16, pp. 321-357, jun 2002. 
[109] G. Tsoumakas and I. Katakis, "Multi-Label Classification: An Overview," in Database Technologies, pp. 309-319, IGI Global, 2009.

[110] F. Fischer, K. Bottinger, H. Xiao, C. Stransky, Y. Acar, M. Backes, and S. Fahl, "Stack Overflow Considered Harmful? The Impact of Copy\&Paste on Android Application Security," in 2017 IEEE Symposium on Security and Privacy (SP), pp. 121-136, IEEE, may 2017.

[111] CodeProject, "https://www.codeproject.com/." (accessed: 2021-08-18).

[112] P. P. Pârtachi, S. K. Dash, M. Allamanis, and E. T. Barr, "Flexeme: Untangling commits using lexical flows," ESEC/FSE 2020 - Proceedings of the 28th ACM Joint Meeting European Software Engineering Conference and Symposium on the Foundations of Software Engineering, pp. 63-74, 2020.

[113] S. O. public network terms of service, "https://stackoverflow.com/legal/termsof-service-licensing." (accessed: 2021-08-20). 


\title{
Appendix A
}

\section{A.1 IClones Configuration Results}

\author{
A.2 Models' Results
}




\begin{tabular}{|c|c|c|c|c|c|}
\hline Configuration & Java & JS & PHP & Py & Avg \\
\hline $\begin{array}{l}\text { Min Block }=0 \\
\text { Min Clone }=5\end{array}$ & $72.92 \%$ & $79.17 \%$ & $77.08 \%$ & $79.17 \%$ & $77.08 \%$ \\
\hline $\begin{array}{l}\text { Min Block }=5 \\
\text { Min Clone }=5\end{array}$ & $72.92 \%$ & $79.17 \%$ & $77.08 \%$ & $79.17 \%$ & $77.08 \%$ \\
\hline $\begin{aligned} \text { Min Block } & =5 \\
\text { Min Clone } & =10\end{aligned}$ & $39.58 \%$ & $39.58 \%$ & $47.92 \%$ & $50.00 \%$ & $44.27 \%$ \\
\hline $\begin{array}{c}\text { Min Block }=10 \\
\text { Min Clone }=5\end{array}$ & $39.58 \%$ & $37.50 \%$ & $45.83 \%$ & $43.75 \%$ & $41.67 \%$ \\
\hline $\begin{array}{l}\text { Min Block }=10 \\
\text { Min Clone }=10\end{array}$ & $39.58 \%$ & $37.50 \%$ & $45.83 \%$ & $43.75 \%$ & $41.67 \%$ \\
\hline $\begin{array}{l}\text { Min Block }=10 \\
\text { Min Clone }=15\end{array}$ & $29.17 \%$ & $31.25 \%$ & $37.50 \%$ & $35.42 \%$ & $33.33 \%$ \\
\hline $\begin{array}{l}\text { Min Block }=10 \\
\text { Min Clone }=20\end{array}$ & $29.17 \%$ & $27.08 \%$ & $37.50 \%$ & $31.25 \%$ & $31.25 \%$ \\
\hline $\begin{aligned} \text { Min Block } & =15 \\
\text { Min Clone } & =5\end{aligned}$ & $29.17 \%$ & $31.25 \%$ & $33.33 \%$ & $35.42 \%$ & $32.29 \%$ \\
\hline $\begin{array}{l}\text { Min Block }=15 \\
\text { Min Clone }=10\end{array}$ & $29.17 \%$ & $31.25 \%$ & $33.33 \%$ & $35.42 \%$ & $32.29 \%$ \\
\hline $\begin{array}{l}\text { Min Block }=15 \\
\text { Min Clone }=15\end{array}$ & $29.17 \%$ & $31.25 \%$ & $33.33 \%$ & $35.42 \%$ & $32.29 \%$ \\
\hline $\begin{array}{l}\text { Min Block }=15 \\
\text { Min Clone }=20\end{array}$ & $27.08 \%$ & $27.08 \%$ & $31.25 \%$ & $31.25 \%$ & $29.17 \%$ \\
\hline $\begin{aligned} \text { Min Block } & =20 \\
\text { Min Clone } & =5\end{aligned}$ & $22.92 \%$ & $27.08 \%$ & $31.25 \%$ & $27.08 \%$ & $27.08 \%$ \\
\hline $\begin{array}{l}\text { Min Block }=20 \\
\text { Min Clone }=10\end{array}$ & $22.92 \%$ & $27.08 \%$ & $31.25 \%$ & $27.08 \%$ & $27.08 \%$ \\
\hline $\begin{array}{l}\text { Min Block }=20 \\
\text { Min Clone }=15\end{array}$ & $22.92 \%$ & $27.08 \%$ & $31.25 \%$ & $27.08 \%$ & $27.08 \%$ \\
\hline $\begin{aligned} \text { Min Block } & =20 \\
\text { Min Clone } & =20\end{aligned}$ & $22.92 \%$ & $27.08 \%$ & $31.25 \%$ & $27.08 \%$ & $27.08 \%$ \\
\hline
\end{tabular}

Table A.1: ICLONES results for different configuration over selected samples. 


\begin{tabular}{|c|c|c|c|c|c|c|c|}
\hline Feature Set & Model & Precision & Recall & F1-Score & Hamming Loss & Accuracy & Exec. Time (s) \\
\hline \multirow{11}{*}{ Code2Vec } & br_GNB & $39.5 \%$ & $60.8 \%$ & $47.9 \%$ & $36.5 \%$ & $48.6 \%$ & 4.6 \\
\hline & br_LR & $78.3 \%$ & $69.7 \%$ & $73.7 \%$ & $13.7 \%$ & $75.7 \%$ & 8.9 \\
\hline & br_RF & $91.1 \%$ & $87.3 \%$ & $89.2 \%$ & $5.9 \%$ & $89.4 \%$ & 421.5 \\
\hline & lp_GNB & $45.7 \%$ & $55.7 \%$ & $50.2 \%$ & $30.5 \%$ & $50.9 \%$ & 6.1 \\
\hline & lp_LR & $78.3 \%$ & $71.8 \%$ & $74.9 \%$ & $13.3 \%$ & $78.1 \%$ & 18.9 \\
\hline & lp_RF & $90.5 \%$ & $87.7 \%$ & $89.1 \%$ & $5.9 \%$ & $90.1 \%$ & 126.2 \\
\hline & mlknn & $85.4 \%$ & $80.1 \%$ & $82.7 \%$ & $9.3 \%$ & $82.5 \%$ & 245.2 \\
\hline & brknn & $87.3 \%$ & $77.2 \%$ & $81.9 \%$ & $9.4 \%$ & $79.2 \%$ & 64.6 \\
\hline & lspc_GNB & $39.5 \%$ & $60.8 \%$ & $47.9 \%$ & $36.5 \%$ & $48.6 \%$ & 5.2 \\
\hline & lspc_LR & $78.3 \%$ & $69.7 \%$ & $73.7 \%$ & $13.7 \%$ & $75.7 \%$ & 10.9 \\
\hline & lspc_RF & $91.1 \%$ & $87.3 \%$ & $89.2 \%$ & $5.8 \%$ & $89.4 \%$ & 432.0 \\
\hline \multirow{11}{*}{ Word2Vec } & br_GNB & $35.0 \%$ & $57.7 \%$ & $43.5 \%$ & $41.3 \%$ & $43.5 \%$ & 7.6 \\
\hline & br_LR & $77.8 \%$ & $69.7 \%$ & $73.5 \%$ & $13.8 \%$ & $74.4 \%$ & 34.0 \\
\hline & br_RF & $91.2 \%$ & $87.5 \%$ & $89.3 \%$ & $5.8 \%$ & $89.5 \%$ & 573.4 \\
\hline & lp_GNB & $41.7 \%$ & $50.2 \%$ & $45.6 \%$ & $33.1 \%$ & $44.7 \%$ & 6.8 \\
\hline & lp_LR & $78.1 \%$ & $71.9 \%$ & $74.9 \%$ & $13.3 \%$ & $78.0 \%$ & 24.8 \\
\hline & lp_RF & $90.5 \%$ & $88.0 \%$ & $89.2 \%$ & $5.9 \%$ & $90.2 \%$ & 174.6 \\
\hline & mlknn & $86.9 \%$ & $79.2 \%$ & $82.9 \%$ & $9.0 \%$ & $82.0 \%$ & 294.7 \\
\hline & brknn & $87.5 \%$ & $77.2 \%$ & $82.1 \%$ & $9.3 \%$ & $79.2 \%$ & 65.0 \\
\hline & lspc_GNB & $35.0 \%$ & $57.7 \%$ & $43.5 \%$ & $41.3 \%$ & $43.5 \%$ & 8.3 \\
\hline & lspc_LR & $77.8 \%$ & $69.7 \%$ & $73.5 \%$ & $13.8 \%$ & $74.4 \%$ & 31.0 \\
\hline & lspc_RF & $91.2 \%$ & $87.5 \%$ & $89.3 \%$ & $5.8 \%$ & $89.4 \%$ & 515.0 \\
\hline \multirow{11}{*}{ TF-IDF } & br_GNB & $39.6 \%$ & $63.0 \%$ & $48.6 \%$ & $36.8 \%$ & $35.0 \%$ & 3.1 \\
\hline & br_LR & $78.0 \%$ & $69.0 \%$ & $73.2 \%$ & $13.9 \%$ & $75.2 \%$ & 6.2 \\
\hline & br_RF & $90.8 \%$ & $87.8 \%$ & $89.3 \%$ & $5.8 \%$ & $89.3 \%$ & 187.9 \\
\hline & lp_GNB & $26.8 \%$ & $36.7 \%$ & $31.0 \%$ & $45.1 \%$ & $27.1 \%$ & 3.5 \\
\hline & lp_LR & $77.5 \%$ & $70.7 \%$ & $74.0 \%$ & $13.7 \%$ & $77.3 \%$ & 15.1 \\
\hline & lp_RF & $90.3 \%$ & $88.2 \%$ & $89.2 \%$ & $5.9 \%$ & $89.9 \%$ & 55.6 \\
\hline & mlknn & $85.4 \%$ & $80.2 \%$ & $82.7 \%$ & $9.3 \%$ & $82.8 \%$ & 235.5 \\
\hline & brknn & $86.9 \%$ & $77.5 \%$ & $81.9 \%$ & $9.4 \%$ & $79.8 \%$ & 47.9 \\
\hline & lspc_GNB & $39.6 \%$ & $63.0 \%$ & $48.6 \%$ & $36.8 \%$ & $35.0 \%$ & 3.8 \\
\hline & lspc_LR & $78.0 \%$ & $69.0 \%$ & $73.2 \%$ & $13.9 \%$ & $75.2 \%$ & 6.8 \\
\hline & lspc_RF & $90.9 \%$ & $87.7 \%$ & $89.3 \%$ & $5.8 \%$ & $89.3 \%$ & 190.5 \\
\hline \multirow{11}{*}{ Statistical } & br_GNB & $65.7 \%$ & $71.4 \%$ & $68.4 \%$ & $18.2 \%$ & $65.2 \%$ & 0.7 \\
\hline & br_LR & $78.2 \%$ & $70.4 \%$ & $74.1 \%$ & $13.6 \%$ & $76.5 \%$ & 2.3 \\
\hline & br_RF & $93.3 \%$ & $89.1 \%$ & $91.2 \%$ & $4.8 \%$ & $90.3 \%$ & 56.1 \\
\hline & lp_GNB & $71.6 \%$ & $69.5 \%$ & $70.5 \%$ & $16.0 \%$ & $72.6 \%$ & 3.9 \\
\hline & lp_LR & $77.8 \%$ & $71.2 \%$ & $74.4 \%$ & $13.5 \%$ & $77.6 \%$ & 8.9 \\
\hline & lp_RF & $92.1 \%$ & $89.6 \%$ & $90.8 \%$ & $5.0 \%$ & $91.3 \%$ & 17.7 \\
\hline & mlknn & $88.3 \%$ & $80.8 \%$ & $84.4 \%$ & $8.2 \%$ & $82.7 \%$ & 72.3 \\
\hline & brknn & $88.3 \%$ & $80.8 \%$ & $84.4 \%$ & $8.2 \%$ & $82.7 \%$ & 15.2 \\
\hline & lspc_GNB & $65.7 \%$ & $71.4 \%$ & $68.4 \%$ & $18.2 \%$ & $65.2 \%$ & 2.7 \\
\hline & lspc_LR & $78.2 \%$ & $70.4 \%$ & $74.1 \%$ & $13.6 \%$ & $76.5 \%$ & 3.8 \\
\hline & lspc_RF & $93.4 \%$ & $88.8 \%$ & $91.1 \%$ & $4.8 \%$ & $90.2 \%$ & 55.0 \\
\hline
\end{tabular}

Table A.2: Precision, recall, F1-score, hamming loss, accuracy, and execution time over OriginalDS. 


\begin{tabular}{|c|c|c|c|c|c|c|c|}
\hline Feature Set & Model & Precision & Recall & F1-Score & Hamming Loss & Accuracy & Exec. Time (s) \\
\hline \multirow{11}{*}{ Code2Vec } & br_GNB & $45.0 \%$ & $53.9 \%$ & $49.0 \%$ & $30.9 \%$ & $30.3 \%$ & 5.9 \\
\hline & br_LR & $66.8 \%$ & $54.2 \%$ & $59.8 \%$ & $20.1 \%$ & $52.2 \%$ & 7.5 \\
\hline & br_RF & $90.4 \%$ & $87.1 \%$ & $88.7 \%$ & $6.1 \%$ & $87.9 \%$ & 365.5 \\
\hline & lp_GNB & $33.0 \%$ & $46.3 \%$ & $38.5 \%$ & $40.8 \%$ & $38.2 \%$ & 4.9 \\
\hline & lp_LR & $71.3 \%$ & $70.8 \%$ & $71.0 \%$ & $15.9 \%$ & $74.1 \%$ & 16.6 \\
\hline & lp_RF & $89.3 \%$ & $88.3 \%$ & $88.8 \%$ & $6.1 \%$ & $89.5 \%$ & 102.6 \\
\hline & mlknn & $76.1 \%$ & $77.3 \%$ & $76.7 \%$ & $13.0 \%$ & $75.5 \%$ & 218.5 \\
\hline & brknn & $76.0 \%$ & $72.7 \%$ & $74.3 \%$ & $13.9 \%$ & $70.4 \%$ & 44.1 \\
\hline & lspc_GNB & $45.0 \%$ & $53.9 \%$ & $49.0 \%$ & $30.9 \%$ & $30.3 \%$ & 4.5 \\
\hline & lspc_LR & $66.8 \%$ & $54.2 \%$ & $59.8 \%$ & $20.1 \%$ & $52.2 \%$ & 8.2 \\
\hline & lspc_RF & $90.3 \%$ & $87.2 \%$ & $88.8 \%$ & $6.1 \%$ & $87.9 \%$ & 360.1 \\
\hline \multirow{11}{*}{ Word2Vec } & br_GNB & $36.4 \%$ & $55.2 \%$ & $43.8 \%$ & $39.0 \%$ & $35.1 \%$ & 7.4 \\
\hline & br_LR & $65.7 \%$ & $55.4 \%$ & $60.1 \%$ & $20.3 \%$ & $52.6 \%$ & 25.0 \\
\hline & br_RF & $90.3 \%$ & $87.1 \%$ & $88.7 \%$ & $6.1 \%$ & $87.5 \%$ & 453.4 \\
\hline & lp_GNB & $33.3 \%$ & $44.5 \%$ & $38.1 \%$ & $39.9 \%$ & $36.6 \%$ & 5.5 \\
\hline & lp_LR & $69.3 \%$ & $69.8 \%$ & $69.6 \%$ & $16.8 \%$ & $72.7 \%$ & 20.3 \\
\hline & lp_RF & $89.3 \%$ & $88.6 \%$ & $88.9 \%$ & $6.1 \%$ & $89.6 \%$ & 127.5 \\
\hline & mlknn & $76.1 \%$ & $81.6 \%$ & $78.7 \%$ & $12.2 \%$ & $76.3 \%$ & 217.8 \\
\hline & brknn & $76.1 \%$ & $72.8 \%$ & $74.4 \%$ & $13.8 \%$ & $70.3 \%$ & 47.4 \\
\hline & lspc_GNB & $36.4 \%$ & $55.2 \%$ & $43.8 \%$ & $39.0 \%$ & $35.1 \%$ & 8.1 \\
\hline & lspc_LR & $65.7 \%$ & $55.4 \%$ & $60.1 \%$ & $20.3 \%$ & $52.6 \%$ & 28.2 \\
\hline & lspc_RF & $90.2 \%$ & $87.1 \%$ & $88.6 \%$ & $6.2 \%$ & $87.4 \%$ & 437.5 \\
\hline \multirow{11}{*}{ TF-IDF } & br_GNB & $35.7 \%$ & $58.6 \%$ & $44.3 \%$ & $40.5 \%$ & $27.1 \%$ & 2.8 \\
\hline & br_LR & $66.8 \%$ & $54.8 \%$ & $60.2 \%$ & $20.0 \%$ & $53.3 \%$ & 4.9 \\
\hline & br_RF & $90.4 \%$ & $87.6 \%$ & $89.0 \%$ & $6.0 \%$ & $88.2 \%$ & 241.6 \\
\hline & lp_GNB & $23.3 \%$ & $32.3 \%$ & $27.1 \%$ & $48.0 \%$ & $23.2 \%$ & 3.3 \\
\hline & lp_LR & $72.2 \%$ & $70.9 \%$ & $71.5 \%$ & $15.6 \%$ & $74.2 \%$ & 13.6 \\
\hline & lp_RF & $89.4 \%$ & $88.5 \%$ & $88.9 \%$ & $6.1 \%$ & $89.4 \%$ & 62.1 \\
\hline & mlknn & $75.4 \%$ & $78.2 \%$ & $76.8 \%$ & $13.0 \%$ & $75.8 \%$ & 213.2 \\
\hline & brknn & $76.8 \%$ & $73.4 \%$ & $75.0 \%$ & $13.5 \%$ & $71.4 \%$ & 38.1 \\
\hline & lspc_GNB & $35.7 \%$ & $58.6 \%$ & $44.3 \%$ & $40.5 \%$ & $27.1 \%$ & 3.6 \\
\hline & lspc_LR & $66.8 \%$ & $54.8 \%$ & $60.2 \%$ & $20.0 \%$ & $53.3 \%$ & 5.5 \\
\hline & lspc_RF & $90.4 \%$ & $87.4 \%$ & $88.9 \%$ & $6.0 \%$ & $88.1 \%$ & 244.3 \\
\hline \multirow{11}{*}{ Statistical } & br_GNB & $40.7 \%$ & $75.4 \%$ & $52.8 \%$ & $37.2 \%$ & $1.2 \%$ & 0.8 \\
\hline & br_LR & $79.8 \%$ & $64.9 \%$ & $71.6 \%$ & $14.2 \%$ & $68.8 \%$ & 2.8 \\
\hline & br_RF & $91.9 \%$ & $88.3 \%$ & $90.0 \%$ & $5.4 \%$ & $87.8 \%$ & 67.5 \\
\hline & lp_GNB & $69.6 \%$ & $68.9 \%$ & $69.2 \%$ & $16.9 \%$ & $72.0 \%$ & 1.9 \\
\hline & lp_LR & $77.1 \%$ & $72.6 \%$ & $74.8 \%$ & $13.5 \%$ & $77.2 \%$ & 9.0 \\
\hline & $l_{p}$ RF & $91.1 \%$ & $90.6 \%$ & $90.8 \%$ & $5.0 \%$ & $90.9 \%$ & 23.2 \\
\hline & mlknn & $79.7 \%$ & $81.4 \%$ & $80.5 \%$ & $10.9 \%$ & $79.0 \%$ & 84.1 \\
\hline & brknn & $83.6 \%$ & $79.5 \%$ & $81.5 \%$ & $10.0 \%$ & $78.4 \%$ & 14.0 \\
\hline & lspc_GNB & $40.7 \%$ & $75.4 \%$ & $52.8 \%$ & $37.2 \%$ & $1.2 \%$ & 4.1 \\
\hline & lspc_LR & $79.8 \%$ & $64.9 \%$ & $71.6 \%$ & $14.2 \%$ & $68.8 \%$ & 5.2 \\
\hline & lspc_RF & $92.0 \%$ & $88.3 \%$ & $90.1 \%$ & $5.3 \%$ & $87.8 \%$ & 79.5 \\
\hline
\end{tabular}

Table A.3: Precision, recall, F1-score, hamming loss, accuracy, and execution time over BalancedDS1. 


\begin{tabular}{|c|c|c|c|c|c|c|c|}
\hline Feature Set & Model & Precision & Recall & F1-Score & Hamming Loss & Accuracy & Exec. Time (s) \\
\hline \multirow{11}{*}{ Code2Vec } & br_GNB & $41.4 \%$ & $51.9 \%$ & $46.1 \%$ & $33.6 \%$ & $21.7 \%$ & 5.8 \\
\hline & br_LR & $62.7 \%$ & $55.3 \%$ & $58.7 \%$ & $21.4 \%$ & $51.6 \%$ & 11.7 \\
\hline & br_RF & $90.3 \%$ & $88.0 \%$ & $89.1 \%$ & $5.9 \%$ & $88.6 \%$ & 564.3 \\
\hline & lp_GNB & $29.0 \%$ & $43.3 \%$ & $34.7 \%$ & $44.9 \%$ & $34.3 \%$ & 6.4 \\
\hline & lp_LR & $69.9 \%$ & $70.4 \%$ & $70.2 \%$ & $16.5 \%$ & $73.5 \%$ & 22.3 \\
\hline & lp_RF & $89.5 \%$ & $88.6 \%$ & $89.1 \%$ & $6.0 \%$ & $89.7 \%$ & 156.6 \\
\hline & mlknn & $76.5 \%$ & $80.4 \%$ & $78.4 \%$ & $12.2 \%$ & $78.2 \%$ & 393.8 \\
\hline & brknn & $76.7 \%$ & $77.0 \%$ & $76.8 \%$ & $12.8 \%$ & $74.4 \%$ & 77.0 \\
\hline & lspc_GNB & $41.4 \%$ & $51.9 \%$ & $46.1 \%$ & $33.6 \%$ & $21.7 \%$ & 13.6 \\
\hline & lspc_LR & $62.7 \%$ & $55.3 \%$ & $58.7 \%$ & $21.4 \%$ & $51.6 \%$ & 12.4 \\
\hline & lspc_RF & $90.3 \%$ & $88.0 \%$ & $89.1 \%$ & $5.9 \%$ & $88.6 \%$ & 575.7 \\
\hline \multirow{11}{*}{ Word2Vec } & br_GNB & $36.6 \%$ & $52.2 \%$ & $43.1 \%$ & $38.1 \%$ & $28.8 \%$ & 7.9 \\
\hline & br_LR & $62.7 \%$ & $57.0 \%$ & $59.7 \%$ & $21.2 \%$ & $52.9 \%$ & 40.0 \\
\hline & br_RF & $90.2 \%$ & $87.9 \%$ & $89.0 \%$ & $6.0 \%$ & $88.3 \%$ & 707.1 \\
\hline & lp_GNB & $30.7 \%$ & $42.8 \%$ & $35.7 \%$ & $42.4 \%$ & $34.0 \%$ & 8.1 \\
\hline & lp_LR & $68.1 \%$ & $70.9 \%$ & $69.4 \%$ & $17.2 \%$ & $72.3 \%$ & 29.0 \\
\hline & lp_RF & $89.4 \%$ & $88.8 \%$ & $89.1 \%$ & $6.0 \%$ & $89.6 \%$ & 192.2 \\
\hline & mlknn & $76.6 \%$ & $80.3 \%$ & $78.4 \%$ & $12.2 \%$ & $77.9 \%$ & 439.1 \\
\hline & brknn & $76.7 \%$ & $76.8 \%$ & $76.7 \%$ & $12.8 \%$ & $73.9 \%$ & 80.9 \\
\hline & lspc_GNB & $36.6 \%$ & $52.2 \%$ & $43.1 \%$ & $38.1 \%$ & $28.8 \%$ & 17.4 \\
\hline & lspc_LR & $62.7 \%$ & $57.0 \%$ & $59.7 \%$ & $21.2 \%$ & $52.9 \%$ & 39.5 \\
\hline & lspc_RF & $90.3 \%$ & $87.7 \%$ & $89.0 \%$ & $6.0 \%$ & $88.2 \%$ & 695.9 \\
\hline \multirow{11}{*}{ TF-IDF } & br_GNB & $35.8 \%$ & $57.9 \%$ & $44.3 \%$ & $40.2 \%$ & $28.4 \%$ & 4.5 \\
\hline & br_LR & $63.2 \%$ & $56.0 \%$ & $59.4 \%$ & $21.1 \%$ & $53.6 \%$ & 13.4 \\
\hline & br_RF & $90.3 \%$ & $88.1 \%$ & $89.2 \%$ & $5.9 \%$ & $89.0 \%$ & 408.5 \\
\hline & lp_GNB & $23.2 \%$ & $32.2 \%$ & $26.9 \%$ & $48.2 \%$ & $22.8 \%$ & 4.7 \\
\hline & lp_LR & $69.2 \%$ & $70.8 \%$ & $70.0 \%$ & $16.8 \%$ & $72.7 \%$ & 19.9 \\
\hline & $l_{p} \_R F$ & $89.6 \%$ & $88.5 \%$ & $89.1 \%$ & $6.0 \%$ & $89.5 \%$ & 100.8 \\
\hline & mlknn & $76.2 \%$ & $81.0 \%$ & $78.5 \%$ & $12.2 \%$ & $78.3 \%$ & 403.1 \\
\hline & brknn & $77.6 \%$ & $77.1 \%$ & $77.4 \%$ & $12.4 \%$ & $75.0 \%$ & 72.5 \\
\hline & lspc_GNB & $35.8 \%$ & $57.9 \%$ & $44.3 \%$ & $40.2 \%$ & $28.4 \%$ & 5.0 \\
\hline & lspc_LR & $63.2 \%$ & $56.0 \%$ & $59.4 \%$ & $21.1 \%$ & $53.6 \%$ & 7.3 \\
\hline & lspc_RF & $90.4 \%$ & $88.0 \%$ & $89.2 \%$ & $5.9 \%$ & $88.8 \%$ & 409.0 \\
\hline \multirow{11}{*}{ Statistical } & br_GNB & $40.4 \%$ & $74.9 \%$ & $52.5 \%$ & $37.4 \%$ & $1.1 \%$ & 1.0 \\
\hline & br_LR & $79.0 \%$ & $65.9 \%$ & $71.9 \%$ & $14.2 \%$ & $69.3 \%$ & 4.5 \\
\hline & br_RF & $92.3 \%$ & $89.2 \%$ & $90.7 \%$ & $5.1 \%$ & $88.9 \%$ & 103.3 \\
\hline & lp_GNB & $69.0 \%$ & $68.4 \%$ & $68.7 \%$ & $17.2 \%$ & $71.4 \%$ & 2.5 \\
\hline & lp_LR & $76.9 \%$ & $72.8 \%$ & $74.8 \%$ & $13.5 \%$ & $77.2 \%$ & 13.1 \\
\hline & lp_RF & $90.8 \%$ & $90.6 \%$ & $90.7 \%$ & $5.1 \%$ & $90.7 \%$ & 31.8 \\
\hline & mlknn & $80.0 \%$ & $82.8 \%$ & $81.4 \%$ & $10.4 \%$ & $80.6 \%$ & 129.7 \\
\hline & brknn & $83.8 \%$ & $81.5 \%$ & $82.6 \%$ & $9.5 \%$ & $80.2 \%$ & 23.3 \\
\hline & lspc_GNB & $40.4 \%$ & $74.9 \%$ & $52.5 \%$ & $37.4 \%$ & $1.1 \%$ & 3.0 \\
\hline & lspc_LR & $79.0 \%$ & $65.9 \%$ & $71.9 \%$ & $14.2 \%$ & $69.3 \%$ & 5.4 \\
\hline & lspc_RF & $92.2 \%$ & $89.3 \%$ & $90.7 \%$ & $5.0 \%$ & $89.0 \%$ & 103.4 \\
\hline
\end{tabular}

Table A.4: Precision, recall, F1-score, hamming loss, accuracy, and execution time over BalancedDS2. 


\begin{tabular}{|c|c|c|c|c|c|c|c|}
\hline Feature Set & Model & Precision & Recall & F1-Score & Hamming Loss & Accuracy & Exec. Time (s) \\
\hline \multirow{11}{*}{ Code2Vec } & br_GNB & $39.0 \%$ & $58.9 \%$ & $47.0 \%$ & $36.7 \%$ & $46.8 \%$ & 2.1 \\
\hline & br_LR & $73.7 \%$ & $50.6 \%$ & $60.0 \%$ & $18.6 \%$ & $51.3 \%$ & 4.5 \\
\hline & br_RF & $90.7 \%$ & $84.9 \%$ & $87.7 \%$ & $6.6 \%$ & $85.8 \%$ & 164.6 \\
\hline & lp_GNB & $43.2 \%$ & $53.6 \%$ & $47.8 \%$ & $32.2 \%$ & $48.1 \%$ & 4.5 \\
\hline & lp_LR & $73.4 \%$ & $70.1 \%$ & $71.7 \%$ & $15.3 \%$ & $74.1 \%$ & 8.2 \\
\hline & lp_RF & $89.1 \%$ & $87.9 \%$ & $88.5 \%$ & $6.3 \%$ & $89.1 \%$ & 50.4 \\
\hline & mlknn & $79.4 \%$ & $72.8 \%$ & $75.9 \%$ & $12.7 \%$ & $72.8 \%$ & 77.3 \\
\hline & brknn & $73.5 \%$ & $62.9 \%$ & $67.8 \%$ & $16.5 \%$ & $61.4 \%$ & 17.8 \\
\hline & lspc_GNB & $39.0 \%$ & $58.9 \%$ & $47.0 \%$ & $36.7 \%$ & $46.8 \%$ & 2.5 \\
\hline & lspc_LR & $73.7 \%$ & $50.6 \%$ & $60.0 \%$ & $18.6 \%$ & $51.3 \%$ & 4.8 \\
\hline & lspc_RF & $90.7 \%$ & $85.0 \%$ & $87.8 \%$ & $6.5 \%$ & $85.8 \%$ & 177.6 \\
\hline \multirow{11}{*}{ Word2Vec } & br_GNB & $34.9 \%$ & $56.6 \%$ & $43.2 \%$ & $41.1 \%$ & $42.1 \%$ & 3.1 \\
\hline & br_LR & $72.4 \%$ & $52.2 \%$ & $60.7 \%$ & $18.7 \%$ & $52.4 \%$ & 17.1 \\
\hline & br_RF & $90.7 \%$ & $84.6 \%$ & $87.5 \%$ & $6.7 \%$ & $85.1 \%$ & 204.8 \\
\hline & lp_GNB & $40.4 \%$ & $49.0 \%$ & $44.3 \%$ & $34.0 \%$ & $43.2 \%$ & 4.4 \\
\hline & lp_LR & $70.8 \%$ & $69.1 \%$ & $69.9 \%$ & $16.4 \%$ & $72.3 \%$ & 11.4 \\
\hline & lp_RF & $89.0 \%$ & $87.9 \%$ & $88.5 \%$ & $6.3 \%$ & $89.1 \%$ & 69.7 \\
\hline & mlknn & $80.6 \%$ & $72.9 \%$ & $76.5 \%$ & $12.3 \%$ & $73.5 \%$ & 89.2 \\
\hline & brknn & $73.8 \%$ & $63.8 \%$ & $68.4 \%$ & $16.2 \%$ & $62.0 \%$ & 24.1 \\
\hline & lspc_GNB & $34.9 \%$ & $56.6 \%$ & $43.2 \%$ & $41.1 \%$ & $42.1 \%$ & 4.3 \\
\hline & lspc_LR & $72.4 \%$ & $52.2 \%$ & $60.7 \%$ & $18.7 \%$ & $52.4 \%$ & 15.4 \\
\hline & lspc_RF & $90.5 \%$ & $84.6 \%$ & $87.4 \%$ & $6.7 \%$ & $85.0 \%$ & 226.4 \\
\hline \multirow{11}{*}{ TF-IDF } & br_GNB & $37.0 \%$ & $61.3 \%$ & $46.2 \%$ & $39.5 \%$ & $27.4 \%$ & 1.8 \\
\hline & br_LR & $74.0 \%$ & $52.1 \%$ & $61.1 \%$ & $18.3 \%$ & $53.5 \%$ & 3.2 \\
\hline & br_RF & $90.2 \%$ & $84.5 \%$ & $87.2 \%$ & $6.8 \%$ & $84.8 \%$ & 77.6 \\
\hline & lp_GNB & $25.6 \%$ & $35.4 \%$ & $29.7 \%$ & $46.1 \%$ & $25.5 \%$ & 2.3 \\
\hline & lp_LR & $73.3 \%$ & $69.4 \%$ & $71.3 \%$ & $15.4 \%$ & $73.4 \%$ & 7.7 \\
\hline & lp_RF & $88.7 \%$ & $88.1 \%$ & $88.4 \%$ & $6.4 \%$ & $88.8 \%$ & 23.5 \\
\hline & mlknn & $77.5 \%$ & $69.6 \%$ & $73.3 \%$ & $14.0 \%$ & $69.5 \%$ & 71.3 \\
\hline & brknn & $75.2 \%$ & $64.6 \%$ & $69.5 \%$ & $15.6 \%$ & $63.5 \%$ & 15.5 \\
\hline & lspc_GNB & $37.0 \%$ & $61.3 \%$ & $46.2 \%$ & $39.5 \%$ & $27.4 \%$ & 2.2 \\
\hline & lspc_LR & $74.0 \%$ & $52.1 \%$ & $61.1 \%$ & $18.3 \%$ & $53.5 \%$ & 3.5 \\
\hline & lspc_RF & $90.2 \%$ & $84.5 \%$ & $87.3 \%$ & $6.8 \%$ & $84.8 \%$ & 77.0 \\
\hline \multirow{11}{*}{ Statistical } & br_GNB & $41.1 \%$ & $74.7 \%$ & $53.0 \%$ & $36.5 \%$ & $1.6 \%$ & 1.7 \\
\hline & br_LR & $81.6 \%$ & $62.8 \%$ & $70.9 \%$ & $14.2 \%$ & $67.4 \%$ & 1.4 \\
\hline & br_RF & $92.4 \%$ & $85.1 \%$ & $88.6 \%$ & $6.1 \%$ & $84.7 \%$ & 27.4 \\
\hline & lp_GNB & $70.7 \%$ & $69.0 \%$ & $69.9 \%$ & $16.4 \%$ & $71.7 \%$ & 1.3 \\
\hline & lp_LR & $77.7 \%$ & $71.6 \%$ & $74.5 \%$ & $13.5 \%$ & $77.2 \%$ & 5.1 \\
\hline & $l_{p} \_R F$ & $91.1 \%$ & $90.0 \%$ & $90.6 \%$ & $5.2 \%$ & $90.5 \%$ & 9.6 \\
\hline & mlknn & $82.1 \%$ & $78.6 \%$ & $80.3 \%$ & $10.6 \%$ & $77.2 \%$ & 32.2 \\
\hline & brknn & $84.2 \%$ & $73.4 \%$ & $78.4 \%$ & $11.1 \%$ & $72.8 \%$ & 7.0 \\
\hline & lspc_GNB & $41.1 \%$ & $74.7 \%$ & $53.0 \%$ & $36.5 \%$ & $1.6 \%$ & 8.5 \\
\hline & lspc_LR & $81.6 \%$ & $62.8 \%$ & $70.9 \%$ & $14.2 \%$ & $67.4 \%$ & 2.1 \\
\hline & lspc_RF & $92.6 \%$ & $85.1 \%$ & $88.7 \%$ & $6.0 \%$ & $84.6 \%$ & 29.4 \\
\hline
\end{tabular}

Table A.5: Precision, recall, F1-score, hamming loss, accuracy, and execution time over BalancedDS3. 


\begin{tabular}{|c|c|c|c|c|c|}
\hline Feature Set & Model & Type I & Type II & Type III & No Clone \\
\hline \multirow{11}{*}{ Code2Vec } & br_GNB & $32.40 \%$ & $19.68 \%$ & $27.93 \%$ & $70.77 \%$ \\
\hline & br_LR & $12.42 \%$ & $11.89 \%$ & $8.68 \%$ & $87.16 \%$ \\
\hline & br_RF & $74.71 \%$ & $72.91 \%$ & $75.05 \%$ & $94.39 \%$ \\
\hline & lp_GNB & $35.04 \%$ & $20.54 \%$ & $30.12 \%$ & $69.68 \%$ \\
\hline & lp_LR & $20.21 \%$ & $16.26 \%$ & $16.41 \%$ & $87.52 \%$ \\
\hline & lp_RF & $74.95 \%$ & $72.27 \%$ & $75.30 \%$ & $94.24 \%$ \\
\hline & mlknn & $60.83 \%$ & $54.90 \%$ & $60.03 \%$ & $91.01 \%$ \\
\hline & brknn & $60.83 \%$ & $54.90 \%$ & $60.03 \%$ & $90.27 \%$ \\
\hline & lspc_GNB & $32.40 \%$ & $19.68 \%$ & $27.93 \%$ & $70.77 \%$ \\
\hline & lspc_LR & $12.42 \%$ & $11.89 \%$ & $8.68 \%$ & $87.16 \%$ \\
\hline & lspc_RF & $75.17 \%$ & $73.08 \%$ & $74.87 \%$ & $94.38 \%$ \\
\hline \multirow{11}{*}{ Word2Vec } & br_GNB & $31.47 \%$ & $18.01 \%$ & $26.65 \%$ & $66.39 \%$ \\
\hline & br_LR & $20.22 \%$ & $16.28 \%$ & $10.31 \%$ & $86.67 \%$ \\
\hline & br_RF & $76.08 \%$ & $72.42 \%$ & $75.57 \%$ & $94.43 \%$ \\
\hline & lp_GNB & $32.76 \%$ & $20.02 \%$ & $31.32 \%$ & $63.17 \%$ \\
\hline & lp_LR & $25.13 \%$ & $15.27 \%$ & $18.06 \%$ & $87.39 \%$ \\
\hline & lp_RF & $75.95 \%$ & $72.16 \%$ & $\mathbf{7 5 . 9 8 \%}$ & $94.30 \%$ \\
\hline & mlknn & $62.42 \%$ & $49.16 \%$ & $56.24 \%$ & $91.32 \%$ \\
\hline & brknn & $62.42 \%$ & $54.88 \%$ & $59.97 \%$ & $90.20 \%$ \\
\hline & lspc_GNB & $31.47 \%$ & $18.01 \%$ & $26.65 \%$ & $66.39 \%$ \\
\hline & lspc_LR & $20.22 \%$ & $16.28 \%$ & $10.31 \%$ & $86.67 \%$ \\
\hline & lspc_RF & $75.87 \%$ & $72.82 \%$ & $75.30 \%$ & $94.41 \%$ \\
\hline \multirow{11}{*}{ TF-IDF } & br_GNB & $33.13 \%$ & $18.47 \%$ & $28.85 \%$ & $72.49 \%$ \\
\hline & br_LR & $9.88 \%$ & $5.49 \%$ & $5.20 \%$ & $86.87 \%$ \\
\hline & br_RF & $76.86 \%$ & $71.65 \%$ & $75.46 \%$ & $94.43 \%$ \\
\hline & lp_GNB & $32.06 \%$ & $17.72 \%$ & $29.53 \%$ & $38.04 \%$ \\
\hline & lp_LR & $12.86 \%$ & $11.64 \%$ & $11.65 \%$ & $87.06 \%$ \\
\hline & lp_RF & $76.67 \%$ & $71.70 \%$ & $76.12 \%$ & $94.32 \%$ \\
\hline & mlknn & $60.48 \%$ & $55.68 \%$ & $58.53 \%$ & $91.01 \%$ \\
\hline & brknn & $60.48 \%$ & $55.68 \%$ & $58.53 \%$ & $90.24 \%$ \\
\hline & lspc_GNB & $33.13 \%$ & $18.47 \%$ & $28.85 \%$ & $72.49 \%$ \\
\hline & lspc_LR & $9.88 \%$ & $5.49 \%$ & $5.20 \%$ & $86.87 \%$ \\
\hline & lspc_RF & $76.47 \%$ & $71.92 \%$ & $75.89 \%$ & $94.41 \%$ \\
\hline \multirow{11}{*}{ Statistical } & br_GNB & $16.99 \%$ & $20.03 \%$ & $21.32 \%$ & $86.26 \%$ \\
\hline & br_LR & $13.97 \%$ & $5.03 \%$ & $12.02 \%$ & $87.51 \%$ \\
\hline & br_RF & $80.29 \%$ & $76.53 \%$ & $79.93 \%$ & $95.51 \%$ \\
\hline & lp_GNB & $23.24 \%$ & $21.62 \%$ & $24.72 \%$ & $85.42 \%$ \\
\hline & lp_LR & $18.01 \%$ & $7.97 \%$ & $11.13 \%$ & $87.56 \%$ \\
\hline & lp_RF & $79.54 \%$ & $76.52 \%$ & $79.04 \%$ & $95.13 \%$ \\
\hline & mlknn & $65.72 \%$ & $56.99 \%$ & $63.02 \%$ & $91.98 \%$ \\
\hline & brknn & $65.72 \%$ & $56.99 \%$ & $63.02 \%$ & $91.98 \%$ \\
\hline & lspc_GNB & $16.99 \%$ & $20.03 \%$ & $21.32 \%$ & $86.26 \%$ \\
\hline & lspc_LR & $13.97 \%$ & $5.03 \%$ & $12.02 \%$ & $87.51 \%$ \\
\hline & lspc $R F$ & $80.27 \%$ & $75.59 \%$ & $79.21 \%$ & $95.50 \%$ \\
\hline
\end{tabular}

Table A.6: F1-score results per clone types over OriginalDS. 


\begin{tabular}{|c|c|c|c|c|c|}
\hline Feature Set & Model & Type I & Type II & Type III & No Clone \\
\hline \multirow{11}{*}{ Code2Vec } & br_GNB & $29.43 \%$ & $23.79 \%$ & $30.93 \%$ & $67.16 \%$ \\
\hline & br_LR & $34.14 \%$ & $15.69 \%$ & $36.72 \%$ & $73.67 \%$ \\
\hline & br_RF & $76.57 \%$ & $72.64 \%$ & $75.29 \%$ & $93.97 \%$ \\
\hline & lp_GNB & $31.29 \%$ & $18.14 \%$ & $26.04 \%$ & $56.39 \%$ \\
\hline & lp_LR & $33.10 \%$ & $26.72 \%$ & $34.42 \%$ & $85.10 \%$ \\
\hline & lp_RF & $75.78 \%$ & $72.02 \%$ & $75.04 \%$ & $94.16 \%$ \\
\hline & mlknn & $60.82 \%$ & $52.83 \%$ & $58.55 \%$ & $86.65 \%$ \\
\hline & brknn & $60.82 \%$ & $52.83 \%$ & $58.55 \%$ & $83.36 \%$ \\
\hline & lspc_GNB & $29.43 \%$ & $23.79 \%$ & $30.93 \%$ & $67.16 \%$ \\
\hline & lspc_LR & $34.14 \%$ & $15.69 \%$ & $36.72 \%$ & $73.67 \%$ \\
\hline & lspc_RF & $76.61 \%$ & $73.11 \%$ & $75.62 \%$ & $94.00 \%$ \\
\hline \multirow{11}{*}{ Word2Vec } & br_GNB & $35.11 \%$ & $21.53 \%$ & $27.87 \%$ & $63.19 \%$ \\
\hline & br_LR & $37.27 \%$ & $18.89 \%$ & $37.17 \%$ & $73.55 \%$ \\
\hline & br_RF & $77.25 \%$ & $72.32 \%$ & $75.55 \%$ & $93.94 \%$ \\
\hline & lp_GNB & $30.15 \%$ & $18.94 \%$ & $28.15 \%$ & $54.36 \%$ \\
\hline & lp_LR & $34.41 \%$ & $30.42 \%$ & $34.18 \%$ & $84.16 \%$ \\
\hline & lp_RF & $76.94 \%$ & $71.82 \%$ & $75.18 \%$ & $94.26 \%$ \\
\hline & mlknn & $61.07 \%$ & $53.86 \%$ & $58.26 \%$ & $89.30 \%$ \\
\hline & brknn & $61.07 \%$ & $53.86 \%$ & $58.26 \%$ & $83.49 \%$ \\
\hline & lspc_GNB & $35.11 \%$ & $21.53 \%$ & $27.87 \%$ & $63.19 \%$ \\
\hline & lspc_LR & $37.27 \%$ & $18.89 \%$ & $37.17 \%$ & $73.55 \%$ \\
\hline & lspc_RF & $77.30 \%$ & $72.10 \%$ & $75.64 \%$ & $93.87 \%$ \\
\hline \multirow{11}{*}{ TF-IDF } & br_GNB & $31.18 \%$ & $15.68 \%$ & $27.80 \%$ & $68.34 \%$ \\
\hline & br_LR & $35.86 \%$ & $15.68 \%$ & $35.40 \%$ & $73.49 \%$ \\
\hline & br_RF & $77.20 \%$ & $72.43 \%$ & $75.99 \%$ & $94.20 \%$ \\
\hline & lp_GNB & $30.40 \%$ & $17.06 \%$ & $25.24 \%$ & $31.70 \%$ \\
\hline & lp_LR & $31.59 \%$ & $25.81 \%$ & $33.87 \%$ & $85.39 \%$ \\
\hline & lp_RF & $76.92 \%$ & $72.07 \%$ & $75.48 \%$ & $94.24 \%$ \\
\hline & mlknn & $60.33 \%$ & $50.96 \%$ & $58.48 \%$ & $87.12 \%$ \\
\hline & brknn & $60.33 \%$ & $54.90 \%$ & $58.48 \%$ & $84.05 \%$ \\
\hline & lspc_GNB & $31.18 \%$ & $15.68 \%$ & $27.80 \%$ & $68.34 \%$ \\
\hline & lspc_LR & $35.86 \%$ & $15.68 \%$ & $35.40 \%$ & $73.49 \%$ \\
\hline & lspc_RF & $76.93 \%$ & $72.30 \%$ & $75.28 \%$ & $94.22 \%$ \\
\hline \multirow{11}{*}{ Statistical } & br_GNB & $20.80 \%$ & $11.18 \%$ & $20.86 \%$ & $86.28 \%$ \\
\hline & br_LR & $18.35 \%$ & $8.13 \%$ & $28.54 \%$ & $85.15 \%$ \\
\hline & br_RF & $80.18 \%$ & $75.58 \%$ & $78.83 \%$ & $94.68 \%$ \\
\hline & lp_GNB & $21.02 \%$ & $22.43 \%$ & $20.54 \%$ & $85.22 \%$ \\
\hline & lp_LR & $22.67 \%$ & $16.11 \%$ & $27.76 \%$ & $87.64 \%$ \\
\hline & lp_RF & $80.59 \%$ & $75.70 \%$ & $79.78 \%$ & $95.34 \%$ \\
\hline & mlknn & $65.79 \%$ & $57.23 \%$ & $63.06 \%$ & $89.47 \%$ \\
\hline & brknn & $66.28 \%$ & $58.48 \%$ & $64.56 \%$ & $89.47 \%$ \\
\hline & lspc_GNB & $20.80 \%$ & $11.18 \%$ & $20.86 \%$ & $86.28 \%$ \\
\hline & lspc_LR & $18.35 \%$ & $8.13 \%$ & $28.54 \%$ & $85.15 \%$ \\
\hline & lspc_RF & $80.40 \%$ & $75.34 \%$ & $79.24 \%$ & $94.71 \%$ \\
\hline
\end{tabular}

Table A.7: F1-score results per clone types over BalancedDS1. 


\begin{tabular}{|c|c|c|c|c|c|}
\hline Feature Set & Model & Type I & Type II & Type III & No Clone \\
\hline \multirow{11}{*}{ Code2Vec } & br_GNB & $22.51 \%$ & $12.81 \%$ & $31.75 \%$ & $66.87 \%$ \\
\hline & br_LR & $31.92 \%$ & $14.63 \%$ & $37.41 \%$ & $73.49 \%$ \\
\hline & br_RF & $76.85 \%$ & $71.44 \%$ & $76.07 \%$ & $94.39 \%$ \\
\hline & lp_GNB & $30.09 \%$ & $17.04 \%$ & $24.13 \%$ & $51.52 \%$ \\
\hline & lp_LR & $29.89 \%$ & $24.50 \%$ & $35.06 \%$ & $84.80 \%$ \\
\hline & lp_RF & $76.25 \%$ & $70.38 \%$ & $76.35 \%$ & $94.38 \%$ \\
\hline & mlknn & $62.21 \%$ & $51.07 \%$ & $60.49 \%$ & $88.56 \%$ \\
\hline & brknn & $62.21 \%$ & $51.07 \%$ & $60.49 \%$ & $86.50 \%$ \\
\hline & lspc_GNB & $22.51 \%$ & $12.81 \%$ & $31.75 \%$ & $66.87 \%$ \\
\hline & lspc_LR & $31.92 \%$ & $14.63 \%$ & $37.41 \%$ & $73.49 \%$ \\
\hline & lspc_RF & $76.87 \%$ & $71.21 \%$ & $76.32 \%$ & $94.37 \%$ \\
\hline \multirow{11}{*}{ Word2Vec } & br_GNB & $28.41 \%$ & $22.40 \%$ & $27.15 \%$ & $63.06 \%$ \\
\hline & br_LR & $36.39 \%$ & $19.17 \%$ & $38.31 \%$ & $73.89 \%$ \\
\hline & br_RF & $76.65 \%$ & $71.96 \%$ & $76.11 \%$ & $94.36 \%$ \\
\hline & lp_GNB & $29.37 \%$ & $18.41 \%$ & $27.40 \%$ & $50.98 \%$ \\
\hline & lp_LR & $37.69 \%$ & $27.17 \%$ & $36.90 \%$ & $84.21 \%$ \\
\hline & lp_RF & $76.35 \%$ & $70.75 \%$ & $76.52 \%$ & $94.36 \%$ \\
\hline & mlknn & $62.79 \%$ & $51.81 \%$ & $60.08 \%$ & $88.53 \%$ \\
\hline & brknn & $62.79 \%$ & $51.81 \%$ & $60.08 \%$ & $86.29 \%$ \\
\hline & lspc_GNB & $28.41 \%$ & $22.40 \%$ & $27.15 \%$ & $63.06 \%$ \\
\hline & lspc_LR & $36.39 \%$ & $19.17 \%$ & $38.31 \%$ & $73.89 \%$ \\
\hline & lspc_RF & $76.53 \%$ & $71.81 \%$ & $76.36 \%$ & $94.30 \%$ \\
\hline \multirow{11}{*}{ TF-IDF } & br_GNB & $30.59 \%$ & $15.93 \%$ & $27.91 \%$ & $67.68 \%$ \\
\hline & br_LR & $34.05 \%$ & $16.97 \%$ & $36.14 \%$ & $73.78 \%$ \\
\hline & br_RF & $77.05 \%$ & $70.92 \%$ & $76.22 \%$ & $94.41 \%$ \\
\hline & lp_GNB & $30.03 \%$ & $17.15 \%$ & $25.60 \%$ & $31.16 \%$ \\
\hline & lp_LR & $29.54 \%$ & $27.52 \%$ & $36.55 \%$ & $84.68 \%$ \\
\hline & lp_RF & $76.25 \%$ & $71.30 \%$ & $76.39 \%$ & $94.36 \%$ \\
\hline & mlknn & $61.38 \%$ & $51.02 \%$ & $60.49 \%$ & $88.81 \%$ \\
\hline & brknn & $61.38 \%$ & $53.77 \%$ & $60.49 \%$ & $86.81 \%$ \\
\hline & lspc GNB & $30.59 \%$ & $15.93 \%$ & $27.91 \%$ & $67.68 \%$ \\
\hline & lspc_LR & $34.05 \%$ & $16.97 \%$ & $36.14 \%$ & $73.78 \%$ \\
\hline & lspc_RF & $76.65 \%$ & $71.99 \%$ & $75.88 \%$ & $94.48 \%$ \\
\hline \multirow{11}{*}{ Statistical } & br_GNB & $19.77 \%$ & $10.58 \%$ & $20.74 \%$ & $86.17 \%$ \\
\hline & br_LR & $17.47 \%$ & $8.06 \%$ & $33.36 \%$ & $85.45 \%$ \\
\hline & br_RF & $80.32 \%$ & $74.94 \%$ & $80.28 \%$ & $95.23 \%$ \\
\hline & lp_GNB & $16.56 \%$ & $21.86 \%$ & $22.52 \%$ & $84.99 \%$ \\
\hline & lp_LR & $23.51 \%$ & $17.34 \%$ & $27.79 \%$ & $87.65 \%$ \\
\hline & lp_RF & $79.72 \%$ & $73.96 \%$ & $80.32 \%$ & $95.29 \%$ \\
\hline & mlknn & $66.17 \%$ & $56.27 \%$ & $63.73 \%$ & $90.41 \%$ \\
\hline & brknn & $67.41 \%$ & $57.86 \%$ & $66.92 \%$ & $90.41 \%$ \\
\hline & lspc_GNB & $19.77 \%$ & $10.58 \%$ & $20.74 \%$ & $86.17 \%$ \\
\hline & lspc_LR & $17.47 \%$ & $8.06 \%$ & $33.36 \%$ & $85.45 \%$ \\
\hline & lspc_RF & $80.34 \%$ & $75.50 \%$ & $80.74 \%$ & $95.23 \%$ \\
\hline
\end{tabular}

Table A.8: F1-score results per clone types over BalancedDS2. 


\begin{tabular}{|c|c|c|c|c|c|}
\hline Feature Set & Model & Type I & Type II & Type III & No Clone \\
\hline \multirow{11}{*}{ Code2Vec } & br_GNB & $32.98 \%$ & $20.45 \%$ & $28.77 \%$ & $68.47 \%$ \\
\hline & br_LR & $36.31 \%$ & $16.46 \%$ & $28.24 \%$ & $72.62 \%$ \\
\hline & br_RF & $76.10 \%$ & $72.51 \%$ & $74.11 \%$ & $93.02 \%$ \\
\hline & lp_GNB & $34.13 \%$ & $20.26 \%$ & $29.89 \%$ & $66.94 \%$ \\
\hline & lp_LR & $35.50 \%$ & $33.75 \%$ & $32.14 \%$ & $84.87 \%$ \\
\hline & lp_RF & $76.16 \%$ & $72.16 \%$ & $74.44 \%$ & $93.89 \%$ \\
\hline & mlknn & $57.26 \%$ & $51.92 \%$ & $55.62 \%$ & $85.51 \%$ \\
\hline & brknn & $55.60 \%$ & $51.92 \%$ & $54.19 \%$ & $75.93 \%$ \\
\hline & lspc_GNB & $32.98 \%$ & $20.45 \%$ & $28.77 \%$ & $68.47 \%$ \\
\hline & lspc_LR & $36.31 \%$ & $16.46 \%$ & $28.24 \%$ & $72.62 \%$ \\
\hline & lspc_RF & $76.39 \%$ & $72.78 \%$ & $74.15 \%$ & $92.97 \%$ \\
\hline \multirow{11}{*}{ Word2Vec } & br_GNB & $32.06 \%$ & $18.39 \%$ & $27.36 \%$ & $64.86 \%$ \\
\hline & br_LR & $37.50 \%$ & $16.65 \%$ & $30.12 \%$ & $73.42 \%$ \\
\hline & br_RF & $76.07 \%$ & $72.51 \%$ & $74.67 \%$ & $92.76 \%$ \\
\hline & lp_GNB & $32.56 \%$ & $19.82 \%$ & $31.42 \%$ & $61.43 \%$ \\
\hline & lp_LR & $36.26 \%$ & $34.57 \%$ & $34.92 \%$ & $83.58 \%$ \\
\hline & lp_RF & $76.27 \%$ & $72.12 \%$ & $74.66 \%$ & $93.91 \%$ \\
\hline & mlknn & $58.60 \%$ & $52.91 \%$ & $54.83 \%$ & $85.87 \%$ \\
\hline & brknn & $56.42 \%$ & $53.78 \%$ & $54.19 \%$ & $76.47 \%$ \\
\hline & lspc_GNB & $32.06 \%$ & $18.39 \%$ & $27.36 \%$ & $64.86 \%$ \\
\hline & lspc_LR & $37.50 \%$ & $16.65 \%$ & $30.12 \%$ & $73.42 \%$ \\
\hline & lspc_RF & $76.24 \%$ & $72.70 \%$ & $74.89 \%$ & $92.59 \%$ \\
\hline \multirow{11}{*}{ TF-IDF } & br_GNB & $33.80 \%$ & $17.43 \%$ & $28.11 \%$ & $70.28 \%$ \\
\hline & br_LR & $37.58 \%$ & $19.31 \%$ & $23.00 \%$ & $73.61 \%$ \\
\hline & br_RF & $76.53 \%$ & $71.77 \%$ & $74.33 \%$ & $92.50 \%$ \\
\hline & lp_GNB & $31.78 \%$ & $17.59 \%$ & $29.15 \%$ & $35.30 \%$ \\
\hline & lp_LR & $35.17 \%$ & $32.30 \%$ & $31.93 \%$ & $84.57 \%$ \\
\hline & lp_RF & $76.54 \%$ & $71.85 \%$ & $74.91 \%$ & $93.87 \%$ \\
\hline & mlknn & $56.26 \%$ & $52.52 \%$ & $54.58 \%$ & $82.65 \%$ \\
\hline & brknn & $56.26 \%$ & $52.52 \%$ & $53.17 \%$ & $78.01 \%$ \\
\hline & lspc GNB & $33.80 \%$ & $17.43 \%$ & $28.11 \%$ & $70.28 \%$ \\
\hline & lspc_LR & $37.58 \%$ & $19.31 \%$ & $23.00 \%$ & $73.61 \%$ \\
\hline & lspc_RF & $76.92 \%$ & $72.10 \%$ & $74.25 \%$ & $92.49 \%$ \\
\hline \multirow{11}{*}{ Statistical } & br_GNB & $17.50 \%$ & $11.19 \%$ & $20.82 \%$ & $86.33 \%$ \\
\hline & br_LR & $22.92 \%$ & $7.32 \%$ & $17.26 \%$ & $84.58 \%$ \\
\hline & br_RF & $79.98 \%$ & $75.96 \%$ & $78.51 \%$ & $92.80 \%$ \\
\hline & lp_GNB & $27.22 \%$ & $21.75 \%$ & $25.08 \%$ & $84.93 \%$ \\
\hline & lp_LR & $23.87 \%$ & $18.54 \%$ & $18.35 \%$ & $87.63 \%$ \\
\hline & lp_RF & $80.50 \%$ & $76.21 \%$ & $78.90 \%$ & $95.12 \%$ \\
\hline & mlknn & $64.37 \%$ & $57.84 \%$ & $61.67 \%$ & $88.74 \%$ \\
\hline & brknn & $64.37 \%$ & $57.84 \%$ & $61.98 \%$ & $85.88 \%$ \\
\hline & lspc_GNB & $17.50 \%$ & $11.19 \%$ & $20.82 \%$ & $86.33 \%$ \\
\hline & lspc_LR & $22.92 \%$ & $7.32 \%$ & $17.26 \%$ & $84.58 \%$ \\
\hline & lspc_RF & $80.14 \%$ & $76.53 \%$ & $78.87 \%$ & $92.86 \%$ \\
\hline
\end{tabular}

Table A.9: F1-score results per clone types over BalancedDS3. 